\title{
Thermosensory Transduction Mechanisms in Drosophila melanogaster
}

\author{
Dissertation \\ for the award of the degree \\ "Doctor rerum naturalium" \\ of the Georg-August-Universität Göttingen \\ within the doctoral program Sensory and Motor Neuroscience \\ of the Georg-August University School of Science (GAUSS)
}

Submitted by

Robert Kossen

from Bremen, Germany 


\section{Thesis Committee}

Referee:

Prof. Dr. Martin C. Göpfert

Department of Cellular Neurobiology

Schwann-Schleiden-Research Centre

Julia-Lermontowa Weg 3, 37077 Göttingen

2nd Referee:

Prof. Dr. Ernst A. Wimmer

Department for Developmental Biology

Göttingen Center for Molecular Biosciences (GZMB)

Justus-von-Liebig-Weg 11, 37077 Göttingen

Prof. Dr. Manuela Schmidt

Somatosensory Signaling Group

Max-Planck-Institute for Experimental Medicine

Hermann-Rein-Str. 3, 37075 Göttingen

\section{Members of the Examination Board}

Prof. Dr. Tim Gollisch

Department of Ophthalmology, Sensory Processing in the Retina Group University Medical Center Göttingen

Waldweg 33, 37073 Göttingen

PD Dr. Roland Dosch

Department of Developmental Biochemistry

Justus-von-Liebig-Weg 11, 37077 Göttingen

Dr. Jan Clemens

Neuronal Computation and Behaviour

European Neuroscience Institute Göttingen

Grisebachstr. 5, 37077 Göttingen

Date of oral examination: 28th of August, 2019 
Herewith I declare that I have written this thesis independently and with no other aids and sources than quoted.

Göttingen, July 17 2019, Robert Kossen 


\section{Contents}

Abstract $\quad$ iii

List of Figures iii

List of Tables $\quad$ vi

1 Introduction 1

$1.1 \quad$ TRP ion channels . . . . . . . . . . . . . . . . . . 3

1.2 The Drosophila NOMPC TRP channel . . . . . . . . . . . . . . . . 6

1.3 Thermosensation . . . . . . . . . . . . . . 8

1.3.1 Thermosensation in Drosophila melanogaster . . . . . . . . . . 10

1.3.2 Thermosensation in the arista of Drosophila . . . . . . . . . . . 13

2 Material \& Methods 17

2.1 Fly Husbandry . . . . . . . . . . . . . . . . . . . . . . . 17

2.2 Genetic tools \& mutant flies . . . . . . . . . . . . . . . . . . 17

2.3 Fly lines . . . . . . . . . . . . . . . . . . . . . . . . . . . 19

2.4 In vivo calcium imaging . . . . . . . . . . . . . . . . 20

2.4.1 Temperature stimulation . . . . . . . . . . . . . . . 21

2.4.2 Mechanical stimulation . . . . . . . . . . . . . . 25

2.4.3 Calcium imaging analysis . . . . . . . . . . . . . 25

2.4.3.1 Analysis of temperature stimulated $\mathrm{Ca}^{2+}$ imaging . . . . 26

2.4.3.2 Analysis of mechanically stimulated $\mathrm{Ca}^{2+}$ imaging . . . . 28

2.5 Temperature preference behaviour . . . . . . . . . . . . . . . . 28

2.5.1 Recording of temperature preference behaviour . . . . . . . . 28

2.5.2 Analysis of temperature preference behaviour . . . . . . . . 30

2.6 Microscopy ............................ 31

2.6 .1 Image Acquisition . . . . . . . . . . . . . . . 31

2.6.2 Imaging of Gal4 driven expression patterns . . . . . . . . . . . 31

2.7 Statistical analysis . . . . . . . . . . . . . . . . . . 31 
3.1 nompC is expressed in the hot cells of the arista . . . . . . . . . . . 33

3.2 Hot and cold cells show relative calcium responses to temperature stimuli 36

3.3 Effect of NOMPC on calcium responses in arista neurons . . . . . . . . 45 3.3.1 Mutations of nompC lead to a reduction in $\mathrm{HC}$ response amplitude 45

3.3.2 NompC rescue restores $\mathrm{HC}$ response amplitude . . . . . . . . . . 54

3.3.3 Overexpression of nompC does not significantly alter $\mathrm{HC}$ responses 54

3.4 Arista neurons do not show calcium responses to mechanical deflection . 56

3.5 NOMPC affects temperature preference and tolerance behaviour . . . . . 58

4 Discussion 63

4.1 The response properties of thermosensitive arista neurons . . . . . . . . 64

4.1.1 Arista neurons are relative temperature sensors . . . . . . . . . . 64

4.1.2 Arista neurons show phasic-tonic responses to prolonged temperature changes . . . . . . . . . . . . 65

4.2 NOMPC modulates hot cell responses . . . . . . . . . . . . 66

4.3 nompC mutations alter temperature avoidance behaviour . . . . . . 71

5 References $\quad 75$

References . . . . . . . . . . . . . . . . . . . 75

6 Appendix $\quad 91$

List of Appendix Figures . . . . . . . . . . . . . . . . . . . . . 93

6.1 Appendix: $\mathrm{Ca}^{2+}$ traces sorted by temperature stimulus . . . . . . . . . . 94

6.2 Appendix: $\mathrm{Ca}^{2+}$ traces sorted by fly strains . . . . . . . . . . . . . 98

$\begin{array}{lll}7 & \text { List of abbreviations } & 107\end{array}$

8 Acknowledgements 109

9 Curriculum Vitae 111 


\section{Abstract}

Ambient temperature has an profound effect in the physiology of all animals and accurate evaluation of both external and internal temperatures is therefore an essential factor for fitness and survival. To this end, animals possess specialized cells and organs, which have evolved to obtain accurate information about this crucial factor.

Drosophila melanogaster relies on different thermosenstive neurons to gauge both its internal as well as the external temperature. One thermosensitive organ is located in the arista, a feather like structure protruding from the third segment of the fly's antenna. It has been found that the arista houses six neurons, three of which exhibit excitatory responses to heat (and were therefore called hot cells) and three which are responding to cold (named cold cells). In this study, I characterized the responses properties of the thermosensory organ in the arista and I analyzed the role that the TRP ion channel NOMPC, classically associated with mechanosensation, plays in this particular structure.

Using $\mathrm{Ca}^{2+}$ imaging, I found that the amplitude of temperature evoked $\mathrm{Ca}^{2+}$ responses appears to be determined by the relative change in temperature, rather than the absolute value.

I furthermore found evidence that the TRP channel NOMPC can be found in the hot cell population of arista neurons. Data obtained in this study shows that a knockdown of nompC leads to a reduction in the temperature response amplitude of hot cells. Homozygous nompC null mutants exhibit a stronger reduction in amplitude than heterozygous mutants, hinting at a possible gene dosage effect. The response modulation via NOMPC also affects behaviour, as locomotion experiments in a temperature gradient showed that nompC knockdown mutants show later hot avoidance and have a higher preferred temperature. The effect in both physiological and behavioural experiments could be rescued.

Taken together, the findings of this study show that the thermosensitive neurons in the arista of Drosophila function as relative temperature sensors and that NOMPC serves an important modulatory role for the temperature evoked responses of these neurons. 


\section{List of Figures}

1 Expression of GFP driven by nompC-Gal4 in the antenna of Drosophila . . 2

2 Overview of TRP channel groups and subfamilies . . . . . . . . . . . 4

3 Overview of Drosophila TRP channels .............. 6

4 Structure of the NOMPC TRP channel . . . . . . . . . . . . . 8

5 Overview of Drosophila temperature sensitive neurons . . . . . . . . . . 11

6 Schematic overview of temperature sensitive neurons in the arista . . . . 14

7 Schematic represenation of GCaMP structure and function $\ldots \ldots$. . . . 21

8 Overview of Drosophila preperation for $\mathrm{Ca}^{2+}$ imaging . . . . . . . . . . 22

9 Temperature stimulation control GUI . . . . . . . . . . . . . . . . . . 23

10 Temperature stimuli during $\mathrm{Ca}^{2+}$ imaging experiments . . . . . . . . . . . 24

11 Example of calcium imaging analysis . . . . . . . . . . . . . . . 27

12 Illustration of temperature preference behaviour arena . . . . . . . . . 29

13 Temperature preference correction via IGLOO . . . . . . . . . . . 30

14 Cellular expression pattern of nompc . . . . . . . . . . . . 35

15 Temperature evoked $\mathrm{Ca}^{2+}$ responses of wildtype flies . . . . . . . . . . 37

16 Comparison of $\mathrm{Ca}^{2+}$ responses to different temperature stimulus protocols 39

17 Comparison of $\mathrm{HC} \mathrm{Ca}^{2+}$ responses to temperature steps of same relative

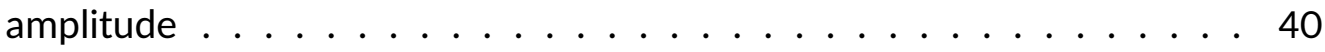

18 Comparison of $\mathrm{HC} \mathrm{Ca}^{2+}$ responses to temperature steps of different relative amplitudes . . . . . . . . . . . . . . . . . . . 41

19 Mean $\mathrm{Ca}^{2+}$ responses to relative temperature steps . . . . . . . . . . . 42

20 Sigmoid fits to relative temperature step responses of wildtype control flies 43

21 Calcium response of arista neurons to prolonged temperature change . . 44

22 Mean $\mathrm{Ca}^{2+}$ responses of hot cells in nompC ${ }^{3}$ mutant, rescue and overexpression flies . . . . . . . . . . . . . . . . . 4 47

23 Mean $\mathrm{Ca}^{2+}$ responses of cold cells in nompC $\mathrm{C}^{3}$ mutant, rescue and overexpression flies . . . . . . . . . . . . . . . . . . 49 49

24 Sigmoid fits to relative temperature step hot cell responses . . . . . . . . 50

25 Sigmoid fits to relative temperature step cold cell responses . . . . . . . 51

26 Comparison of $\Delta \mathrm{F} / \mathrm{F}_{0}$ gain per ${ }^{\circ} \mathrm{C} \ldots \ldots \ldots \ldots$ 
27 Gain of linear fits to hot and cold cell responses . . . . . . . . . . . . . 56

28 Calcium responses to mechanical stimulation of the arista . . . . . . 57

29 Temperature distributions of Drosophila . . . . . . . . . . . . . . . 59

30 Comparison of temperature avoidance and preferences . . . . . . . . . 62 


\section{List of Tables}

1 Drosophila lines \& genotypes . . . . . . . . . . . . . . . . . . . . . . . . 19

1 Drosophila lines \& genotypes (continued) . . . . . . . . . . . . 20 


\section{Chapter 1}

\section{Introduction}

Regulatory systems are vital for basically all processes in living organisms. From the first prokaryotes to highly evolved multicellular organisms, regulatory processes have developed to optimize and control cell metabolism, signalling and development (see for example Junger 2011; Mullur, Liu, and Brent 2014; Partridge, Lopez, and Johnston 1984). We find comparable regulatory processes on any other biological level from cell-organelles up to complex regulatory behaviour of animals. While the scope of regulatory processes changes with the complexity of the underlying system, the general basis appears to stay the same, as the concept of feedback regulation can be found from system such as gene expression (Hepker et al. 1997; Sheen 1994) to proper control of locomotion via proprioreception (Lam and Pearson 2002; Pearson 1995). Regulatory systems, regardless if they are of technical, subcellular or behavioural nature are based on the IPO principle (InputProcessing-Output) (Frank 2013; Partridge, Lopez, and Johnston 1984; Waring 1996), implying that without sensory input, the finest tuned regulatory system is useless.

In order to extract information from the environment, animals rely on different senses as a sort of interface between them and their surroundings. This process is indispensable for the survival and fitness of behaving animals, be it to locate food sources, conspecifics or to avoid harmful circumstances such as extreme environmental conditions or predators. For non-sessile animals, this means that almost all behaviours are at least modulated, if not even motivated, by sensory input (Carlson 1994; Shettleworth 2001).

When thinking about the different senses and sensory modalities (such as vision, gustation, olfaction, temperature sensation, or mechanosensation), it is easy to imagine them as distinct and separate processes. Research, however, has begun to find a steadily increasing number of conserved features, structures and mechanisms between different sensory modalities. These features were not only conserved between different species, but also between the fundamental processes of different senses. This includes factors regarding the development of sensory cells/organs, as for example the Drosophila gene atonal (ato) has been found to be required for the development of both photoreceptors and stretch receptive organs (Caldwell and Eberl 2002; Jarman et al. 1995; Niwa, Hiromi, 
and Okabe 2004).

Shared features also extend into the area of actual sensory transduction, such as the different functions of opsins (primarily associated with the visual sense) in proprioception (Zanini et al. 2018), or the effect of opsins and chemosensors on the sound transduction/hearing in Drosophila (Senthilan et al. 2012). The sharing of transduction molecules in different sensory modalities is widespread in the animal kingdom: In mammals, the TRP channel TRPV-1 has been shown to be responsible for the perception of noxious temperatures, chemicals (such as capsaicin) and pH (Aneiros et al. 2011; Caterina et al. 1997; Salazar et al. 2008). Similarly, the mammalian ion channel TREK-1, which has been found to be a mechanically sensitive channel, can also be activated via chemical ligands, intracellular acidification and even heat (Brohawn, Su, and MacKinnon 2014; Chemin et al. 2005; Maingret, Lauritzen, et al. 2000; Maingret, Patel, et al. 1999; Maingret, Patel, et al. 2000).

The ability to extract information from the environment predates the evolution of distinct sensory organs. Single-celled organisms for example, use ion channels of, as mechanosensors (Sukharev et al. 1994) or cytoplasmatic kinases involved in chemosensation (Frank, Piñas, et al. 2016; Parkinson, Hazelbauer, and Falke 2015; Tu 2013). These findings have given rise to the idea that the different senses and the underlying transduction mechanisms might evolutionary related. the theory suggests a protosensory cell, from which the distinct sensory systems and organs of higher animals evolved and specialized (Niwa, Hiromi, and Okabe 2004; Simpson 2011).

The superfamily of TRP on channels is of special notice in this context, as its members have been found to be implicated in a wide number of sensory processes in many different species (including insects, worms, mice and humans), with certain channels playing a role in more than one sensory pathway/modality (for reviews, see Fowler and Montell 2013; Kadowaki 2015; Pan, Yang, and Reinach 2011; Venkatachalam and Montell 2007).

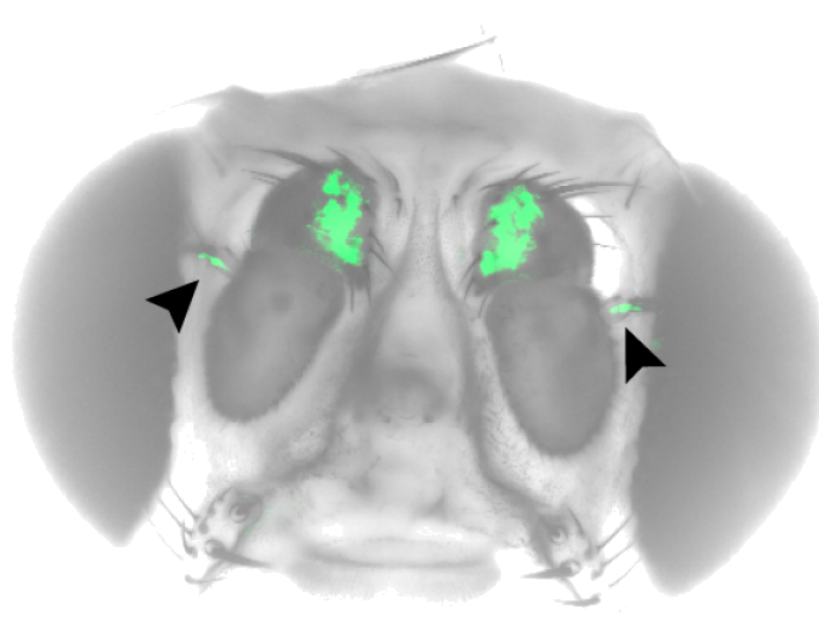

NompC > mCD8-GFP
Figure 1: Expression of GFP driven by nompC-Gal4 in the antenna of Drosophila Overview image of a Drosophila head, expressing the membrane associated fluorescent protein mcd8-GFP under UAS control, driven via nompC-Gal4. Visible flourescence in the second antennal segment, which contains the animals hearing organ (Johnston's organ), and in the base of arista (black arrowheads), which is the location of a set of thermosensitive neurons. Image courtesy of Dr. B.R.H.Geurten. 
In the study presented here, I studied the involvement of the TRP channel NOMPC, a mechanotransducer channel, in the context of a seemingly completely different sensory modality: temperature sensation. Initial studies of nompC expression in our lab found evidence that the TRP channel could be present in a set of neurons in the arista (Figure 1), which have been implicated in temperature sensation (Gallio et al. 2011; Ni, Bronk, et al. 2013).

\subsection{TRP ion channels}

The superfamily of transient receptor potential (TRP) channels encompasses more than 30 , relatively unspecific cation channels. In contrast to many other ion channel families, TRP channels show a surprisingly wide range of ion permeabilities, activation mechanisms and sensory modalities in which they act. Interestingly, some TRP channels can be activated through different mechanisms/modalities, as will be described in detail below, and they are therefore often considered as signal integrators of different stimuli (see for example Venkatachalam and Montell 2007). The importance of TRP channels for the responses to almost all external stimuli and their presence in so many different animal species makes them a compelling research subject when thinking about sensory processes and the underlying molecular mechanisms.

Even though members of the TRP channel family have been found to be involved in many different sensory modalities across a wide range of species, they all share a number of similar structural motifs: These are: six transmembrane domains, with the pore forming region located between the 5th and 6th transmembrane domain, a permeability for cations and the location of both $\mathrm{N}$ - and $\mathrm{C}$-termini of the protein on the intracellular side (Montell 2005; Venkatachalam and Montell 2007).

The different TRP channels have been categorized into two main groups and seven subgroups (or sub-families), based on their role, structural properties and species in which they can be found. All sub-groups/sub-families follow a naming convention, in which a one or two letter abbreviation (usually derived from a phenotype, disease or structural feature, associated with the first found member of the sub-family) follows the TRP initials (Clapham, Montell, et al. 2003; Montell et al. 2002; Pan, Yang, and Reinach 2011).

Shared characteristics of members of the group 1 of TRP channels include a varying number of ankyrin repeats at the N-terminus region (with the exception of the TRPM subsubfamily) and a so-called TRP domain, usually located shortly after the 6th transmembrane domain in the C-terminal direction (an exception to this is TRPA, which does however feature a structurally very similar $\alpha$-helix in the corresponding region, see Paulsen et al. 2015). It has been suggested that these domains play a role in regulating gating properties (including the direct gating of mechanosensory channels), ligand binding and localisation. (Cordero-Morales, Gracheva, and Julius 2011; Gaudet 2008; Hwang, Stearns, and 
Tracey 2012; Lishko et al. 2007; Valdez et al. 2019; Zhang, Cheng, et al. 2015). Group 1 encompasses the sub-families TRPA ("ankyrin"), TRPC ("canonical"), TRPM ("Melastatin"), TRPN ("no mechano-receptor potential C" or short "NompC") and TRPV ("vanilloid").

TRP channels belonging to group 2 are lacking the previously described ankyrin repeats and the TRP domain, and appear to share a strikingly long extracellular span between their first and second transmembrane domains. Group 2 includes the sub-families TRPML ("Mucolipin") and TRPP ("Polycystin").

A further sub-family, called TRPY ("yeast") has been found in Sac-

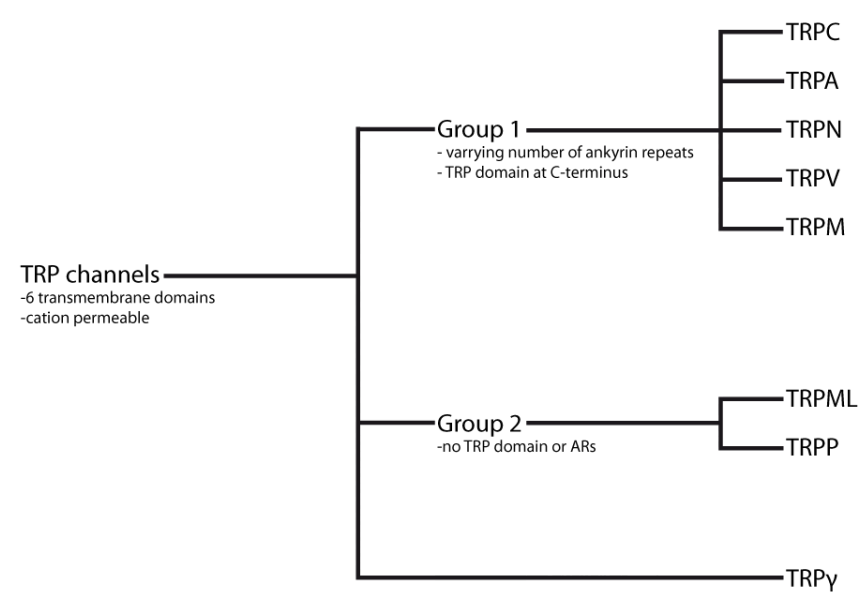
charomyces cerevisiae (Denis and Cyert 2002; Dong, Wang, and Xu 2010; Palmer et al. 2001; Venkatachalam and Montell 2007) and is usually not associated with one of the 2 TRP channel groups described above. Phylogenetic analysis, showed no distinct relation between TRPY and any of the other metazoan TRP channels, suggesting that it emerged after the evolutionary separation

Figure 2: Overview of TRP channel groups and subfamilies Overview over the different TRP channel groups subfamilies. TRP channel subfamilies are organized in 2 main groups. Group 1 is characterized by a varying number of ankyrin repeats at the $\mathrm{N}$ terminus region (with the exception of the TRPM subsubfamily) and a TRP domain, located after 6th transmembrane domain at the C-terminus. Group 2 TRP channels lack these features, but possess a long extracellular span between their first and second transmembrane domain. The TRPY subfamiliy does not belong into any of the two main groups and was so far only found in yeast (Saccharomyces cerevisiae)

of fungi and Metazoans (Cai and Clapham 2011; Kadowaki 2015).

The discovery of different TRP sub-families in apusozoa (Cai and Clapham 2011) and choanoflagellates (Cai 2006; Peng, Shi, and Kadowaki 2015), suggests that the origin of many of the TRP-subfamilies lies within the single-celled ancestors of Metazoa.

As previously mentioned, different TRP channels play a role in wide variety of sensory systems and modalities. These include taste, smell, vision, thermosensation, mechanosensation and hearing (for reviews, see Fowler and Montell 2013; Pan, Yang, and Reinach 2011; Venkatachalam and Montell 2007).

Not only are TRPS found in every sensory modality, some of them are involved in more than one sensory process. Examples are the mammalian TRPM8 channel or the Drosophila 
dTrpA1 channel. Both have been found to be thermosensitive TRP channels, as TRPM 8 is involved in cold sensation (Bautista et al. 2007; McKemy, Neuhausser, and Julius 2002; Nealen et al. 2003; Peier et al. 2002) and dTrpA1 is activated by hot temperatures (Hamada et al. 2008; Rosenzweig, Brennan, et al. 2005). However, it has been described that these channels can also be activated by chemical ligands, with TRPM8 being also activated by menthol and icilin (Andersson, Chase, and Bevan 2004; Dhaka, Viswanath, and Patapoutian 2006; McKemy, Neuhausser, and Julius 2002; Peier et al. 2002). Certain dTrpA1 isoforms react to irritant chemicals, such as hydrogen-peroxide or allyl isothiocyanate (AITC) (Andersson, Gentry, et al. 2008; Kang, Pulver, et al. 2010; Takahashi et al. 2008; Zhong et al. 2012). Additionally, it has been shown that dTrpA1 can even be involved in the indirect sensing of UV light, via its sensitivity to hydrogen-peroxide (Guntur et al. 2015)

The actual activation of TRP channels can occur via different mechanisms. These can be direct activation, or the TRP channel can act as part of a receptor activation system (such as G-protein coupled receptors) or via ligand binding (Clapham, Runnels, and Strübing 2001; Ramsey, Delling, and Clapham 2006). As illustrated by the examples above, the same TRP channel can play a role in the sensing of different stimuli and can be activated via multiple mechanisms. It has also been shown that different activation pathways of the same channel can underlie different modulatory effects: In the mammalian TRPM8 channel, for example, activation via cold or icilin is modulated by intracellular $\mathrm{pH}$, but its activation via menthol does not appear to be modulated this way (Andersson, Chase, and Bevan 2004)

Members of all seven metazoan TRP sub-families have been found in Drosophila, being involved in multiple sensory systems and processes (see Figure 3 for an overview). TRP channels of group 2 in Drosophila have so far not been shown to play a direct role in sensory transduction, though the channel Amo (short for "almost there", also called Pkd2), a member of the TRPP sub-familiy that is required for male fertility/sperm motility, has also been implied in cold sensation (Gao, Ruden, and Lu 2003; Köttgen et al. 2011; Turner et al. 2016; Watnick et al. 2003). Meanwhile, Trpml (only member of the TRPML group in Drosophila) has been shown to be important for calcium homeostasis/transport in lysosomes and maturation of neuromuscular junctions (Wong, Li, et al. 2012; Wong, Palmieri, et al. 2015).

Group 1 TRP channels on the other hand are involved in a multitude of sensory processes in Drosophila: The channels Trp, Trpl and Trp $\gamma$ (belonging to the TRPC sub-family) have been shown to play a role in phototransduction, cold sensation and proprioreception (and by extension fine motor control) (Akitake et al. 2015; Birnbaumer 2009; Rosenzweig, Brennan, et al. 2005). The TRPM sub-family is only represented by one channel in Drosophila, called Trpm, involved in ion homeostasis and larval cold sensation (Georgiev et al. 2010; Hofmann et al. 2010; Turner et al. 2016). 
The TRPV channels in Drosophila are called Nanchung (Nan) and Inactive (Iav) and are implicated in mechanosensensitive processes (such as hearing and gravitation sensing) (Boekhoff-Falk 2005; Gong et al. 2004; Kim, Chung, et al. 2003; Sun et al. 2009; Zhang, Yan, et al. 2013) and thermosensation (Kwon, Shen, et al. 2010). Fruthermore, lav as been implied in synaptic signalling processes (Wong, Chen, et al. 2014) and Nanchung in hygrosensation (Enjin et al. 2016; Liu, Li, et al. 2007). The TRPA subfamily includes four channels in Drosophila, namely dTrpA1, Pain-

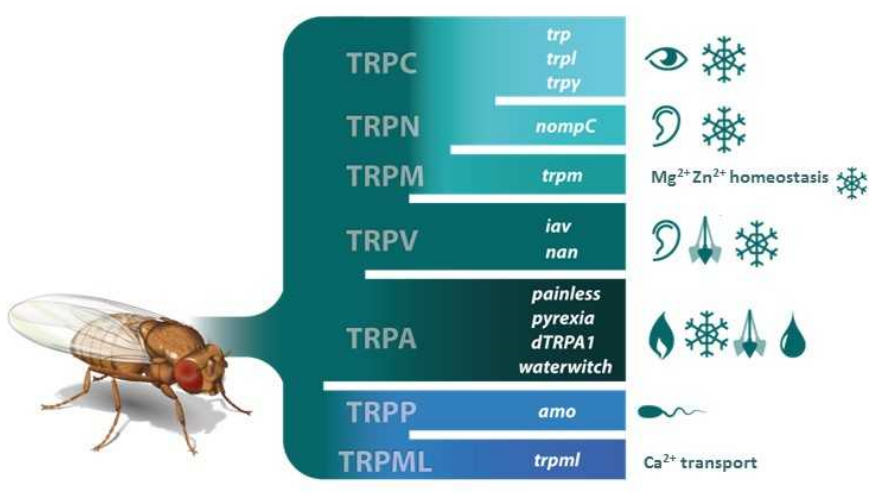
less, Pyrexia and Waterwitch.

The channels of this sub-family play a role in many sensory modalities, including temperature (especially heat) sensation (Hamada et al. 2008; Rosenzweig, Brennan, et al. 2005), thermal and mechanical nociception (Lee, Lee, et al. 2005; Sokabe, Tsujiuchi, et al. 2008; Tracey Jr et al. 2003; Xu et al. 2006), humidity sensing (Liu, Li, et al. 2007), gustation and sensing of irritant chemicals (Andersson, Gentry, et al. 2008; Al-Anzi, Tracey Jr, and Benzer 2006; Kang, Pulver, et al. 2010; Kim, Lee, et al. 2010; Takahashi et al. 2008; Zhong et al. 2012).

Finally, the TRPN sub-family includes a single channel in Drosophila, called nomechanoreceptor potential C (NompC), which has been found to be crucial for multiple senses and processes involving mechanosensation, including hearing, locomotion and gentle touch reception. (Cheng et al. 2010; Effertz, Wiek, and Göpfert 2011; Göpfert, Albert, et al. 2006; Kernan, Cowan, and Zuker 1994; Walker, Willingham, and Zuker 2000; Yan et al. 2013; Zhang, Yan, et al. 2013).

\subsection{The Drosophila NOMPC TRP channel}

The Drosophila TRP channel No mechano-receptor potential C (NompC) is the first discovered member of its sub-family, hence the naming of the group as TRPNOMPC (TRPN). The TRPN sub-family belongs to group 1 of TRP channels, as described in 1.1. Homologues 
of this TRPN channel have been discovered in other species, namely Zebrafish (Danio rerio (Sidi, Friedrich, and Nicolson 2003)), african clawed frog (Xenopus laevis (Shin et al. 2005)), and a nematode (Caenorhabditis elegans (Li et al. 2006)). However, no equivalent channels have been discovered in any mammalian species. So far, no evidence of NOMPC in sensory systems that are not mechanosensitive has been discovered, making its apparent presence in the thermosensory cells of the arista very intriguing.

The NOMPC ion channel contains 29 ankyrin repeats at its $\mathrm{N}$-terminus, connected via a linker domain to the transmembrane domains. It appears that the characteristically long ankyrin repeat domain in NOMPC is required for the mechanically induced gating of NOMPC (Zhang, Cheng, et al. 2015). The C-terminus is characterized by the so called TRP domain (see Figure 4 for an overview).

The NOMPC channel has been shown to be essential for mechanotransduction in a range of sensory systems. It was first discovered that mutations in nompC impaired larval locomotion and touch responses, as well as abolished of mechanoreceptor potentials in sensory bristles of adult Drosophila (hence the name "No mechanorecptor potential"), leading to the idea that NOMPC is a mechanosensory transducer channel (Kernan, Cowan, and Zuker 1994; Walker, Willingham, and Zuker 2000). Soon after, it was shown that NOMPC (together with TRP channels Nan and lav) would be an essential component in Drosophila hearing (Effertz, Wiek, and Göpfert 2011; Göpfert and Robert 2003; Kim, Chung, et al. 2003). It has since been shown that NOMPC plays a role in both larval and adult Drosophila locomotion, mechanosensitivity and sound transduction (Cheng et al. 2010; Yan et al. 2013; Zhang, Yan, et al. 2013). Furthermore, NOMPC was found to be necessary for the mechanosensitivity of certain stretch receptive neurons in the inner and outer labella, facilitating proper feeding behaviour (Zhou et al. 2019).

Additionally, research has found that Drosophila NOMPC appears to play a role in a different sensory modality: The sensing of noxious cold temperatures. nompC was found to be expressed in class III multidendritic neurons of Drosophila larvae. These neurons elicit body contractions in response to noxious cold (Turner et al. 2016). However, the exact way in which NOMPC influences cold sensation remains unclear, as although null mutations of nompC resulted in a reduction of cold evoked contractions, RNAi knockdown of nompC only resulted in a non-significant increase in cold evoked calcium responses. Additionally the TRP channels Amo and Trpm are also found in these neurons (Turner et al. 2016). Class III multidendritic neurons furthermore mediate gentle touch responses, and apparently require NOMPC for this transduction process (Tsubouchi, Caldwell, and Tracey 2012; Yan et al. 2013).

However, as described above, expression studies in our lab found indication of nompC expression in a neuron population inside the arista of adult Drosophila melanogaster (as shown in Figure 1). This group of sensory cells has so far not been shown to be 
A

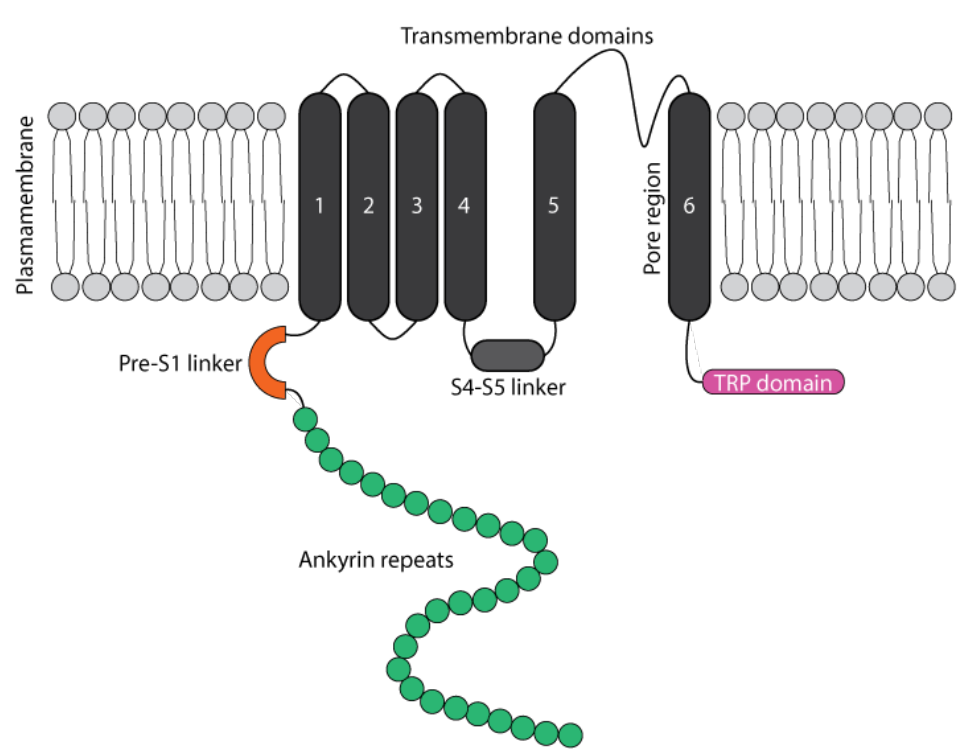

B

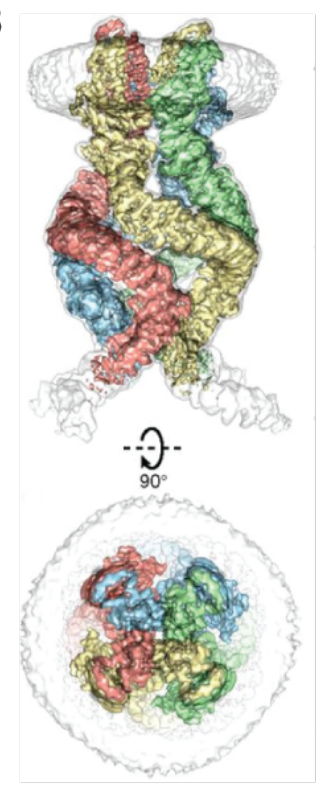

Figure 4: Structure of the NOMPC TRP channel A) Schematic Illustration of the NOMPC channel found in Drosophila. At the N-terminus, NOMPC possesses 29 ankyrin repeats, followed by a linker domain, six transmembrane domains and lastly the TRP domain at the C-Terminus. Both ankyrin repeats and TRP domain are located on the intracellular side of the cellular membrane. Modelling and reconstruction of the tertiary protein structure suggests that the channel pore loop is formed in the region between the 5th and 6th transmembrane domain. B) 3 dimensional reconstruction of the NOMPC ion channel tertiary structure. Illustration taken from Jin et al. 2017.

mechanosensitive, but is involved in Thermosensation (Barbagallo and Garrity 2015; Gallio et al. 2011; Ni, Bronk, et al. 2013).

\subsection{Thermosensation}

Environmental temperatures have a significant impact on almost all physiological processes, and the ability to perceive these environmental factors is paramount to all animals.

When it comes to the molecular basics for thermosensation, insights into channels and activation mechanisms, many facets still remain to be discovered, although research in recent years has advanced this field considerably. As mentioned in 1.1, a number of different TRP channels have in been implicated in thermosensation, in both vertebrates and invertebrates (Dhaka, Viswanath, and Patapoutian 2006; Romanovsky 2007; Vay, Gu, and McNaughton 2012). TRP channels that are involved in thermosensation are often referred to as "thermo-TRPs". However, temperature sensitive channels are not limited to this superfamily, as a range of proteins have been found to play an active role in the transduction of temperature into neuronal signals, belonging to groups such as ionotropic 
receptors, K2P channels or gustatory receptors (Budelli et al. 2019; Ni, Bronk, et al. 2013; Noël et al. 2009; Pereira et al. 2014).

The exact way, by which temperature activates or modulates such temperature sensitive channels/proteins remains largely unknown, although recent studies have begun to investigate and model this process. The relationship between temperature sensing and voltage dependent gating has been extensively discussed in the literature, although no complete model of the relationship has been found (Chowdhury, Jarecki, and Chanda 2014; Latorre, Brauchi, et al. 2007; Voets et al. 2004). The possibility of localized "denaturation", meaning changes in protein tertiary structure induced by temperature have also been discussed (Latorre, Vargas, et al. 2006). Additionally, several structural features have been shown to be crucial for the thermotransduction process (Voets 2012). In different studies for example, almost all structures of the TRP channel TRPV1 have been investigated and considered as thermosesensors, such as the pore turret region and the ankyrin repeat domain (Ladrón-de-Guevara et al. 2019; Voets 2012; Yang et al. 2010). However, the exact way, by which a change in temperature leads to conformation change or gating of the channel remains elusive. Very recent studies, using molecular dynamics simulation, have implied that heat-induced contractions of certain domains of the TRPV1 channel (S2-S3 linker and MPD linker domain) as well as dynamic forming and breaking of hydrogenbonds are major factors for the temperature based gating (Zheng and Wen 2019). Future simulations and experiments will have to show, whether these predicted mechanisms can be confirmed, and whether these are specific features of the TRPV1 channel, or more fundamental properties/mechanisms of temperature gated ion channels.

Understanding the basics of thermosensation is of high interest, as nearly all animal behaviours are informed, modulated or directly motivated by the external and/or internal temperature of the organism (see for example Abram et al. 2017; Briffa, Bridger, and Biro 2013; Kang, Williams, et al. 2010; Steinmetz and Posten 2017). This is especially true in situations of extreme temperature, in which even the endocrine temperature regulation of homeothermic animals quickly reaches its limit and can only partly prevent hyperor hypothermia, respectively (Flouris 2011), or even cause direct damage, resulting from protein denaturation for example.

But even outside of temperature ranges that are immediately harmful to an organism, it is paramount for animals to accurately gauge the surrounding temperatures, as they influence all physiological reactions. This extends beyond immediate effects and into more long-term relevant factors, as exposure to certain temperature ranges can affect adaptive gene expression (such as neuronal dopamine synthesis, see Marija et al. 2019) or even result in longer lasting "memory-effects" of physiological parameters, such as body fat storage (Klepsatel et al. 2016).

While homeothermic animals are less influenced in their bodily functions by external 
temperatures than poikilothermic animals, they still need to take the thermal environmental conditions into account. In fact, certain research suggests, that behavioural thermoregulation is the primary factor for thermal homeostasis in homeothermic organisms, while endocrinic and autonomous thermal-regulaton plays a secondary role (see for example Attia 1984; Flouris 2011).

For poikilotherm animals, this situation is of course even more strict, as the lack of autonomous thermoregulation means they have less latitude when it comes to temperature motivated behaviours: Even comparatively short exposure to unfavourable temperature ranges can have an immediate effect on their physiology, behaviour and ultimately, fitness and survival, thus necessitating distinct and precise behavioural responses (Garrity et al. 2010; Huey, Hertz, and Sinervo 2003; Stevenson 1985).

This influence of ambient temperature is of course even more pronounced in smaller, poikilothermic animals, as a smaller body size results in less "isolating" tissue, meaning central body functions and organs are affected by the external temperature much faster (see for example Heinrich 2013; Sayeed and Benzer 1996).

In the study presented here, thermosensation was investigated in the vinegar fly, Drosophila melanogaster. The demands for accurate thermosensors and fast temperature evoked behaviours in small poikilotherm animals, combined with an anatomically relatively simple nervous system, established genetic tools and mutant lines, make for a convincing case to study these essential sensory processes in the fly.

\subsubsection{Thermosensation in Drosophila melanogaster}

The fly Drosophila possesses a set of different thermosensitive neuronal structures, both in its larval and adult state, sensing both noxious and non-noxious heat and cold (Barbagallo and Garrity 2015). As described above, small, poikilotherm animals such as Drosophila are especially susceptible towards the influence of external temperatures (Garrity et al. 2010; Stevenson 1985), thus necessitating precise and reliable temperature sensation. It has been shown that Drosophila exhibits preference for temperature ranges, which the animal will actively try to pursue, ensuring survival and optimal physiological functions (with the exact temperature ranges varying, depending on factors like the life-cycle stage of the animal or the rearing temperature) (Dillon et al. 2009; Giraldo et al. 2019; Kwon, Shen, et al. 2010; Kwon, Shim, et al. 2008; Sayeed and Benzer 1996). Research has begun to unveil the cellular and molecular basics of how both noxious and non-noxious temperature sensation occurs in the fly.

In the larvae of Drosophila, thermosensors can be found in the head and repeated in each hemisegment of the body. Sensation of noxious cold in the larvae appears to be mediated by class III multidendritic (md) neurons, located in the body wall, which require the TRP channels NompC, Trpm and Amo for proper function (Turner et al. 2016). Non-noxious 


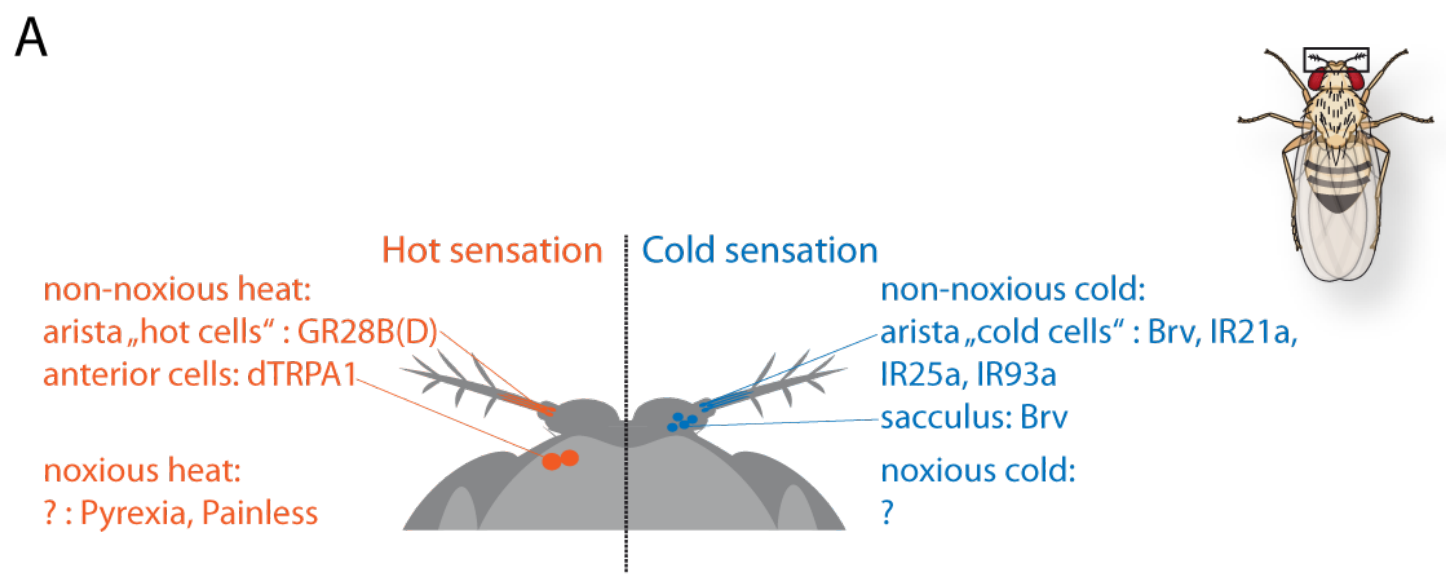

B

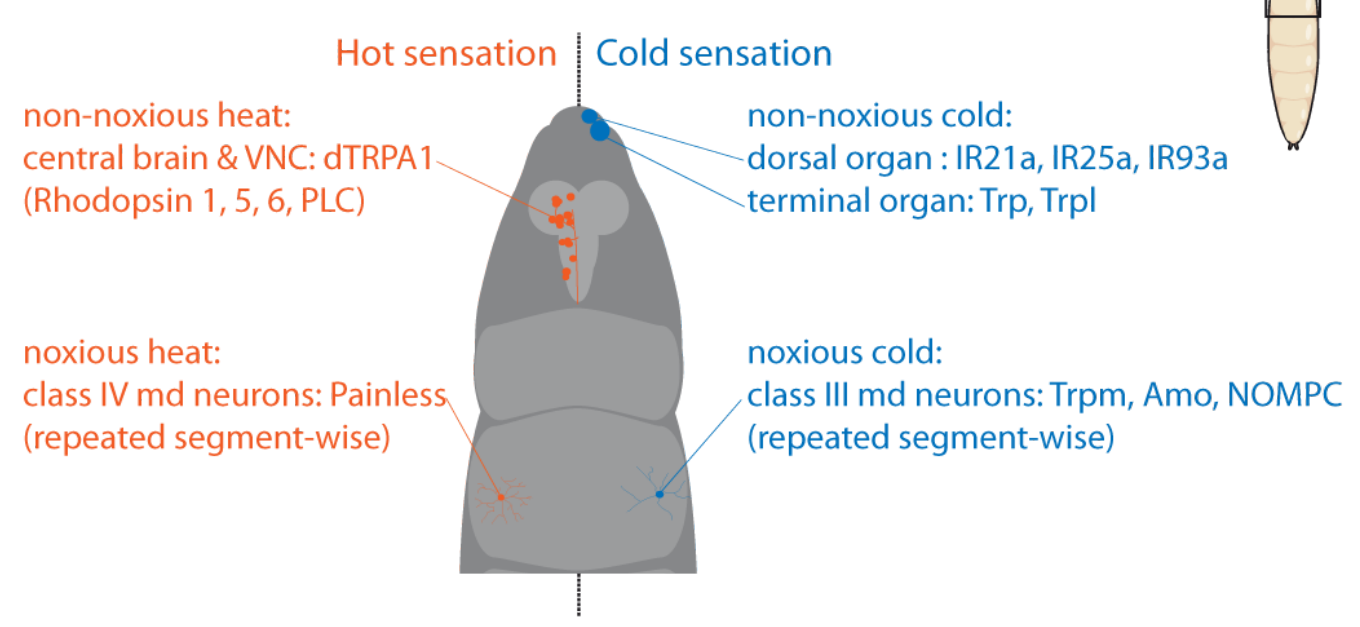

Figure 5: Overview of Drosophila temperature sensitive neurons Schematic overview of temperature sensitive neurons in Drosophila melanogaster, and the proteins that are involved in temperature transduction. Left side shows neurons that have been described as heat sensitive, right side shows neurons described as cold sensitive. A) Temperature sensitive neurons in adult Drosophila. Illustration in upper right corner shows which part of the adult Drosophila is enlarged. Heat sensitive neurons have been found in the arista and the anterior cells of the central brain complex. Cold sensitive neurons were found in the arista and the sacculus of the third antennal segment. It is as of yet unclear, which neuronal structures are responsible for noxious heat and cold sensation. B) Temperature sensitive neurons in Drosophila larvae. Illustration in upper right corner shows which part of the larvae is shown enlarged in grey. Heat sensitive neurons have been found in the central brain complex, ventral nerve cord (VNC) and the multidendritic (md) neurons of the body wall. Cold sensitive neurons have been found in the dorsal and terminal organ in the head and the md neurons close to the body wall (which can be found in each abdominal hemisegment). Larvae and adult fly illustration adapted after Dr. C. Spalthoff, Dr B.R.H. Geurten and Dr. D. Giraldo 
cold sensation has been ascribed to two organs located in the larvaes head: the terminal organ ganglion, requiring the TRP channels Trp and Trpl (Liu, Yermolaieva, et al. 2003; Rosenzweig, Kang, and Garrity 2008), as well as the dorsal organ ganglion, requiring the ionotropic receptors Ir21a, Ir25a and Ir93a (Klein et al. 2015; Knecht et al. 2016; Ni, Klein, et al. 2016).

Larval heat sensation on the other hand was found to be mainly mediated by sensory neurons in the central brain, ventral nerve chord and body wall regions of Drosophila larvae (Liu, Yermolaieva, et al. 2003; Rosenzweig, Brennan, et al. 2005): the TRP channel dTrpA1 was found to be present in a set of neurons in the central brain, the corpus cardiacum and the ventral nerve chord neurons and has been shown to be necessary for warm avoidance (Luo, Shen, and Montell 2017; Rosenzweig, Brennan, et al. 2005). Surprisingly, it was found that dTrpA1 mediated avoidance of lower temperatures (around the $20^{\circ} \mathrm{C}$ mark) is affected by the rhodopsins 1,5 and 6 , as well as phospholipase $\mathrm{C}$ (Kwon, Shim, et al. 2008; Shen et al. 2011; Sokabe, Chen, et al. 2016), proteins classically associated with phototransduction/vision (for a review, see Montell 2012). The exact way, by which these players in warm-transduction interact is still unclear, although it has been suggested that dTrpA1 might act as a thermosensor and the rhodopsin pathway might fulfill a modulatory role (Barbagallo and Garrity 2015; Kwon, Shim, et al. 2008; Shen et al. 2011). This is especially interesting, as functions of opsins beyond direct sensory transduction have been uncovered: Research suggests that ATP independent translocation of phospholipases across cellular membranes (flippase activity) might be vital to develop and maintain proper structure of certain sensory neurons (Ahmad et al. 2007; Giraldo Sanchez 2018; Kumar and Ready 1995; Menon et al. 2011).

Noxious heat sensation has been attributed to so-called class IV md neurons (also found close to the larval body wall) and requires the TRP channel Painless (Sokabe, Tsujiuchi, et al. 2008; Tracey Jr et al. 2003).

Additionally, the chordotonal organs of the Drosophila larvae (stretch receptive organs located close to the animals body wall) were implied to play a role in temperature sensation (Kwon, Shen, et al. 2010; Liu, Yermolaieva, et al. 2003). However, recent research did not show temperature sensitivity of the pentameric chordotonal organ (which was shown to express brivido1) or an effect of brivido1 mutations on larval temperature sensation (Giraldo Sanchez 2018). If and how exactly larval chordotonal organs play a role in thermosensation remains to be determined.

Thermosensation in adult Drosophila has so far been found to be localized to great extend in the head of the animal. The general temperature preference of the fly appears to be driven by the anterior cells, a set of neurons found in the brain, which are responsive towards warm temperatures and require dTrpA1 for this process (Hamada et al. 2008). These anterior cells furthermore seem to act as integrating interneuons for a, as of yet unknown, set of temperature sensor neurons. This hypothesis is corroborated by the fact 
that these neurons show a dTrpA1 independent activity to higher temperatures, which seem to require the TRP channel Pyrexia (Barbagallo and Garrity 2015; Tang et al. 2013). Pyrexia also appears to play a role in the resistance against noxious heat, although its role in the actual sensing of of noxious temperatures has been debated (Lee, Lee, et al. 2005; Neely et al. 2011).

The sensing of noxious temperatures in general remains an understudied topic in adult Drosophila so far. While the TRP channels dTrpA1 and Painless are thought to be involved in the sensing of noxious hot temperatures, the actual cells in which these processes take place remain elusive (Neely et al. 2011; Xu et al. 2006). Noxious cold sensation and its cellular and molecular mechanisms remain unknown (Barbagallo and Garrity 2015).

A set of neurons found in the sacculus, within the third antennal segment has been shown to be involved in the sensing of inocous cold temperatures, with its signals being integrated with the information of other cold sensing neurons of the antenna, in higher order brain regions (Gallio et al. 2011). Lastly, a group of thermosensitive neurons has been found in the arista of Drosophila. The arista itself is a feather-like structure, protruding from the third antennal segment of Drosophilas antenna. It is known that the arista plays an important role in hearing, as deflections of the arista (by sound or wind for example) rotate the third antennal segment, in turn mechanically stimulating stretch receptive neurons inside the second antennal segment (Johnston's organ) (Caldwell and Eberl 2002; Göpfert and Robert 2001; Göpfert and Robert 2002). This mechanosensory function is however not the only sensory process in which the arista is involved: Studies have found a set of six neurons, located inside the arista, to be temperature sensitive. These cells have been described to be necessary for temperature driven behaviours, triggered by both hot and cold, non-noxious temperatures (Budelli et al. 2019; Gallio et al. 2011; Ni, Bronk, et al. 2013).

\subsubsection{Thermosensation in the arista of Drosophila}

The arista of Drosophila houses a population of six neurons which have been shown to be thermosensitive and necessary for certain temperature evoked behaviours (Gallio et al. 2011; Ni, Bronk, et al. 2013; Ni, Klein, et al. 2016). When studying the morpholgy of the arista neurons, one can find the cell bodies located at the proximal base of the arista structure and a single dendrite per cell projecting along the length of the arista (Foelix, Stocker, and Steinbrecht 1989). The axons of the arista neurons project through the third, second and first antennal segment, into the central brain complex of the fly, targeting distinct glomerolus like structures in the posterior antennal lobe (PAL) (also referred to as the proximal antennal protocerebrum (PAP)) area (Frank, Jouandet, et al. 2015; Gallio et al. 2011). 


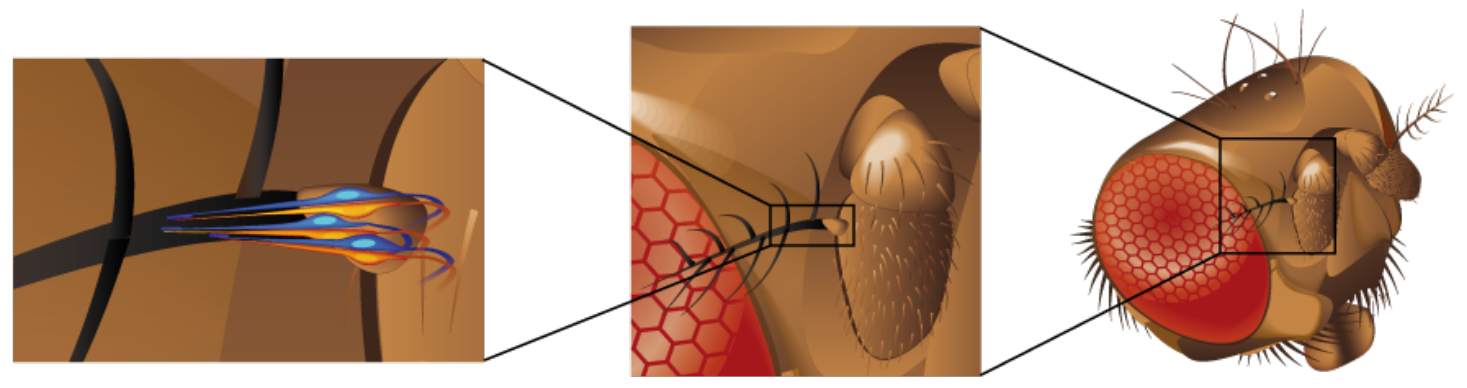

Figure 6: Schematic overview of temperature sensitive neurons in the arista Illustration of the localisation and structure of the temperature sensitive neuron population in the arista of Drosophila. Right of the image shows the whole head of a Drosophila, middle the antennal region enlarged, left shows the lower part of the arista, with temperature sensing neurons inside the arista. Cold cell population shown in blue, hot cells in orange. Cell body of the neurons is located towards the proximal base of the arista, dendrite projects along the axis into the arista. Axons of both cold and hot cells project through the third, second and first antennal segment into the brain. Illustration altered after Dr. B.R.H.Geurten

The six neurons in the arista are temperature sensitive (Gallio et al. 2011; Ni, Bronk, et al. 2013) and their localization at the most distal part of the Drosophila antenna suggests a function as primarily external temperature sensor. The neurons of the arista can be subdivided into two sub-groups with three cells each, that can be distinguished by their functionality: One group appears to be activated by increases in temperature, and was therefore named hot cells $(\mathrm{HC})$, while the other group exhibits activation via cold stimuli and is therefore referred to as cold cells (CC) (Gallio et al. 2011; Ni, Bronk, et al. 2013). Cold cell dendrites show a unique morphological feature, as their endings form distinct, lamellar structures (Budelli et al. 2019; Foelix, Stocker, and Steinbrecht 1989), with the spacing of these lamella supported by so-called bossy orthogonal surface substructures (BOSS) (Steinbrecht 1989). Similar structures are apparently absent from the dendrites of HCs.

Recent studies have begun to unravel the molecular basics for temperature transduction in the $\mathrm{HC}$ and CC population, although several aspects remain poorly understood. Gallio et al. found that cold-evoked calcium responses in the $\mathrm{CC}$ population as well as cold avoidance behaviour was reduced in mutants of the gene brivido (brv)(Gallio et al. 2011). In this context, it has recently been discussed, whether the Drosophila proteins Brivido1-3 (Brv1-3) are members of the TRPP channel family (Gallio et al. 2011), although the Brivido proteins possess 10 transmembrane domains (Fowler and Montell 2013). This aspect has led to the omission of Brv1-3 from some recent lists of TRP channels (Fowler and Montell 2013). Additionally, newer studies involving electrophysiological recordings of the arista were unable to identify a distinct effect of brv mutations on the spiking rate or amplitude of CCs (Budelli et al. 2019). In the same study, Budelli et al. collected evidence that three 
ionotropic receptors (IRs) are not only involved in the temperature evoked responses of $\mathrm{CCs}$, but are also required for proper morphogenesis of the dendritic region and its previously described lamellar structures (Budelli et al. 2019). The three ionotropic receptors are namely IR21a, IR25a and IR93a. As mentioned earlier, these same IRs have previously been found to be important for cold sensation in larvae. It is thought that IR25a and IR93a serve a more regulatory, co-receptor role (and act as such also in other sensory modalites, such as humidity sensing, see for example: Enjin et al. 2016; Kim and Wang 2016), while IR21a acts as specific cooling sensor (Knecht et al. 2016; Ni, Klein, et al. 2016).

The HC group of arista neurons has been shown to express a gene for a gustatory receptor, called gr28b.d. GR28B(D) seems to act as a relatively unspecific cation channel and mutations in this gene result in a impairment of negative thermotactic behaviour in Drosophila (Ni, Bronk, et al. 2013, for a review see Montell 2013). The study performed by $\mathrm{Ni}$ et al. showed that functional GR28B(D) is necessary for the rapid response towards steep warmth gradients (gradient in the described experiment was at least $5^{\circ} \mathrm{C}$ per $\mathrm{cm}$ )(Ni, Bronk, et al. 2013). Additionally, the misexression of the gustatory receptor gene has been found to confer warm sensitivity to a variety of previously not temperature sensitive cells (Mishra et al. 2018; Ni, Bronk, et al. 2013). This clearly suggests that GR28B(D) serves as thermotransducing protein in the HCs of the arista.

The two cell groups appear to connect to distinct glomeruli in the PAL. Additionally, the glomerolus targeted by the projections of the $\mathrm{HC}$ population appears to also receive input from $d \operatorname{TrpA} 1$ expressing neurons, which have been described as internal warm temperature sensing cells (Gallio et al. 2011; Hamada et al. 2008), while a set of cold sensitive neurons found in the sacculus of Drosophilas third antennal segment also projects to the PAL region (Liu, Mazor, and Wilson 2015). From the PAL, projection neurons then relay the temperature information to higher order brain areas (Florence and Reiser 2015; Frank, Jouandet, et al. 2015).

Research in the recent years has postulated that the reason for multiple sensory systems involved in the sensing of non-noxious temperatures is a functional seperation: Whereas more internal temperature sensing neurons, such as the dTrpA1 positive cells, are necessary for the development of longterm temperature preferences and responses to longer temperature exposure, which reaches the body core, the more peripheral sensors, such as the arista neurons, are not involved in these processes ( $\mathrm{Ni}$, Bronk, et al. 2013). On the other hand, the peripheral sensors found in the antenna are necessary for the sensing of steep temperature steps in the ambient temperature. Taken together, it appears that while dTrpA1 serves as sensor for the registration of internal body temperature, the positioning of $\mathrm{HC}$ and $\mathrm{CC}$ populations in the most distal part of the antenna, the arista, points to a function as external temperature sensor. Interestingly, transduction of temperature in these cells involves proteins belonging to the groups of ionotropic and gustatory receptors, but members of the TRP channel superfamily have so far not 
been shown to be involved in these thermosensors (if excluding the Brv channels for the reasons mentioned above). This is especially surprising, given the otherwise widespread implementation of TRP channels in sensory systems, including thermosensation, in other structures of the fly and other animals. In this study, I present first evidence of the presence of a TRP channel in the HCs of the arista: the TRPN channel NOMPC. The goal of my PhD thesis was to determine the influence of NOMPC on the sensory physiology of the $\mathrm{HCs}$ and on temperature preferrence and avoidance behaviour. 


\section{Chapter 2}

\section{Material \& Methods}

\subsection{Fly Husbandry}

Drosophila were kept in small vials on fly food (fresh yeast: 71,43g/L; sugar: 71,43g/L; flour: 25,71g/L; salt: 2,86g/L; propionic acid: 4,29 mL/L, apple juice 142,86mL/L; agarose $8,57 \mathrm{~g} / \mathrm{L})$. Vials were closed using mite proof plugs. All fly lines were raised and kept at 25 ${ }^{\circ} \mathrm{C}$ (to avoid systematic alterations of prefered temperatures, see Giraldo et al. 2019) and $60 \%$ humidity, in a 12h/12h light/dark cycle.

Flies with a homozygous nomp $\mathrm{C}^{3}$ mutation could not be kept in normal fly vials, as the severe impairment to mechanosensation makes it impossible for the flies to perform coordinated movements, including basic locomotion and flying. These mutant animals would stick to the food at the bottom of the vial and were therefore collected after ecclosion and put into a Petri dish, containing slightly moist tissue paper. The mutant flies survived several days in this dish.

\subsection{Genetic tools \& mutant flies}

Different established genetic methods were used during this study. Established mutant lines for the nompC gene in this study include nompC $C^{3}$, which carries a mutation resulting in a premature stop codon in the anykrin repeat area of NOMPC (Cheng et al. 2010; Walker, Willingham, and Zuker 2000), leading to a complete loss of detectable protein (see for example Liang et al. 2011), as well as a line carrying the nompC f00914 mutation, in which a piggyBac construct (Thibault et al. 2004) insertion into the gene leads to lower expression rates of the gene, and therefore reduced amount of the protein (Lee, Moon, et al. 2010; Sun et al. 2009).

In addition to fly lines carrying specific mutations in genes of interest, two binary expression systems were employed: The Gal4/UAS system (Brand and Perrimon 1993) as well 
as the LexA/LexAop system (Lai and Lee 2006). Both systems allow for the expression of one or more desired genes in a cell or tissue specific manner. These systems were used here in order to: (i) examine the expression pattern of certain genes using genetically expressed fluorophores, (ii) rescue mutant phenotypes by re-introducing functional copies of a gene, (iii) ablate cells using apoptotic factors Hid (Grether et al. 1995) and Rpr (White et al. 1994) and (iv) to express the calcium indicator GCaMP6m (Chen et al. 2013). The advantage of using two different expression system lies in the fact that they do not directly influence each other, allowing for two different genes to be expressed in different cell population within the same fly line. 


\subsection{Fly lines}

\begin{tabular}{|c|c|c|}
\hline Strain & Genotype & Source \\
\hline Cantons & $+/+;+/+;+/+$ & BDSC64349 \\
\hline $\begin{array}{l}\text { white nSybLexA LexAop } \\
\text { GCaMP6m }\end{array}$ & $\begin{array}{l}w^{1118} / w^{1118} ; n \text { nSybLexA/CyO ; } \\
\text { LexAop GCaMP6m/TM6C }\end{array}$ & Provided by Dr. C. Spalthoff \\
\hline nompC $C^{3}$, nSybLexA & $\begin{array}{l}+/+\quad ; \quad \text { nompC } C^{3} / C y O ; n S y- \\
b L \operatorname{exA} / T M 6 B\end{array}$ & Provided by A. Adden \\
\hline $\begin{array}{ll}\text { nompC } & 3, \\
\text { GCaMP6m } & \text { LexAop }\end{array}$ & $\begin{array}{l}\text { +/+ ; nompC }{ }^{3} / \text { CyO ; LexAop } \\
\text { GCaMP6m/TM6B }\end{array}$ & $\begin{array}{l}\text { Obtained by crossing dou- } \\
\text { ble balanced nompC }{ }^{3} \text { line } \\
\text { with bloomington LexAop } \\
\text { GCaMP6m line (BDSC44276) }\end{array}$ \\
\hline $\begin{array}{l}\text { nompC }{ }^{3}, \quad \text { recombined } \\
\text { imaging }\end{array}$ & $\begin{array}{l}+/+ \text {; nompC }{ }^{3} / C y O \text {; nSybLexA, } \\
\text { LexAop GCaMP6m/TM6B }\end{array}$ & $\begin{array}{l}\text { Obtained by homologous re- } \\
\text { combination of the nompC3, } \\
\text { nSybLexA and nompC3 } \\
\text { LexAop GCaMP6m lines }\end{array}$ \\
\hline $\begin{array}{l}\text { nompC-Gal4, } \quad \text { nompc } C^{3} \\
\text { background }\end{array}$ & $\begin{array}{l}+/+; \text { nompC }{ }^{3} / C y O ; \text { nompC- } \\
\text { Gal4/TM6B }\end{array}$ & $\begin{array}{l}\text { Obtained by crossing nompC } \\
\text { rescue parental line with dou- } \\
\text { ble balanced nompC } 3 \text { line }\end{array}$ \\
\hline nompc $C^{f 00914}$, nSybLexA & $\begin{array}{l}+/+; \operatorname{nompC} C^{f 00914} / C y O ; n S y- \\
\text { bLexA/TM6B }\end{array}$ & Provided A. Adden \\
\hline $\begin{array}{l}\text { nompC }{ }^{f 00914}, \quad \text { LexAop } \\
\text { GCaMP6m }\end{array}$ & $\begin{array}{l}+/+; \text { nompC } C^{f 00914} / \text { CyO ; LexAop } \\
\text { GCaMP6m/TM6B }\end{array}$ & $\begin{array}{l}\text { Obtained by crossing double } \\
\text { balanced nompC foo914 line } \\
\text { with Bloomington LexAop } \\
\text { GCaMP6m line (BDSC44276) }\end{array}$ \\
\hline nompC rescue (parental) & $\begin{array}{l}+/+; \text { nompC }{ }^{3}, \text { UASnompC- } \\
\text { GFP/CYO ; nompC-Gal4/TM6B }\end{array}$ & Provided by Li Cheng (UCSF) \\
\hline $\begin{array}{l}\text { nompC Overexpression } \\
\text { (parental) }\end{array}$ & $\begin{array}{l}+/+; \text { UASnompC-GFP/CyO ; } \\
\text { nompC-Gal4/nompC-Gal4 }\end{array}$ & Provided by Li Cheng (UCSF) \\
\hline $\begin{array}{l}\text { nompC-Gal4 UAS-mcd8- } \\
\text { GFP }\end{array}$ & $\begin{array}{l}+/+; \text { CyO/sp ; nompC-gal4, } \\
\text { UAS-mcd8-GFP/nompC-Gal4, } \\
\text { UAS-mcd8-GFP }\end{array}$ & Provided by Li Cheng (UCSF) \\
\hline HC-Gal4 & $w^{*} ;$ PGawBhot-cell ; +/TM6b & $\begin{array}{l}\text { Provided by M. Gallio (North- } \\
\text { western University) }\end{array}$ \\
\hline
\end{tabular}

Table 1: Drosophila lines \& genotypes 


\begin{tabular}{|c|c|c|}
\hline CC-Gal4 & $\begin{array}{l}y^{*} W^{*} \quad ; \quad+/+ \\
P\{G a w B\} N P 4486 / T M 6, \quad P\{U A S- \\
\text { lacZ.UW23-1\}UW23-1 }\end{array}$ & Kyoto Stock center: 104694 \\
\hline UAS-mcd8-GFP & $\begin{array}{l}w^{*} \quad ; \quad+/+ \\
\text { Py[+t7.7]w[+mC]=20XUAS- } \\
\text { IVS-mCD8::GFPattP2 }\end{array}$ & BDSC32194 \\
\hline UAS-hid,rpr & UAS-hid,rpr ; +/+ ; +/+ & Provided by John Nambu \\
\hline
\end{tabular}

Table 1: Drosophila lines \& genotypes (continued)

\subsection{In vivo calcium imaging}

Rise in intracellular calcium concentration as a measure of neuronal activity was used to assess responses of arista neurons. All calcium imaging experiments were performed on adult Drosophila, 2-6 days after eclosion. Both male and female flies were used, as pilot studies in our lab showed no systemic difference in the neuronal $\mathrm{Ca}^{2+}$ responses of the arista neurons. A Zeiss Axio Examiner.D1 microscope (Carl Zeiss AG, Oberkochen, Germany) was used and all movies were recorded using a Orca Flash 4.0 camera (Hamamatsu Orca Flash 4.0 V2, C11440-22CU, Hamamatsu Photonics K.K., Hamamatsu, Japan) and the Micro-Manager (https://micro-manager.org) software kit. Unless otherwise noted, all movies in this setup were recorded using a Zeiss 40X water immersion obejective (Zeiss Objective W "Plan-Apochromat" 40x/1.0 DIC M27). Movies were recorded with a frame rate of 10 frames per second ( $\mathrm{fps}$ ). The genetically encoded calcium indicator (GECI) GCaMP6m (Chen et al. 2013) was used and expressed in a nearly panneuronal fashion, using the LexA-LexAop binary expression system (Lai and Lee 2006) and a neuronalsynaptobrevin promotor (nSyb-LexA). Since the arista only houses the 6 thermosensitive neurons that are being investigated in this study, the panneuronal expression did not interfere with detecting the desired neurons, as the target neurons do not occlude each other too often. In most experiments, one or more neurons were clearly separable.

The GCaMP family of GECIs consist of an circularly mutated EGFP molecule, fused with a calmodulin (CaM) and the so-called M13 domain (a peptide sequence obtained from the enzyme myosin light-chain kinase). $\mathrm{Ca}^{2+}$ can bind to the $\mathrm{CaM}$, leading to a conformational change in the EGFP, which increases its fluorescence (see Figure 7) (Akerboom et al. 2009; Nakai, Ohkura, and Imoto 2001; Wang et al. 2008).

Over the course of this study, a total of 954 flies were measured in this $\mathrm{Ca}^{2+}$ setup. Of these, a total of 226 animals yielded recordings, resulting in a total of 495 cell signals measured.

It should be noted that by panneuronal expression, $\mathrm{Ca}^{2+}$ responses of hot and cold cells 


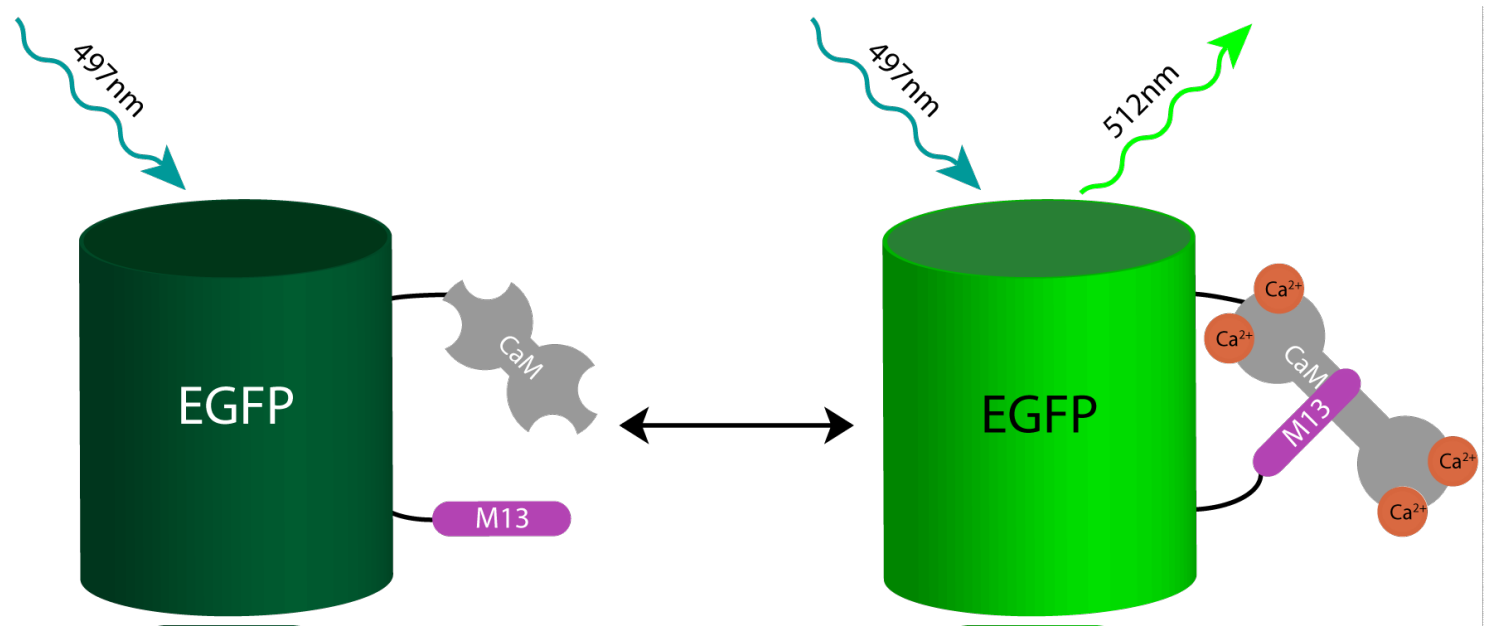

Figure 7: Schematic representation of the structure and function of a GCaMP molecule GCaMP consists of an EGFP, a clamodulin (CaM) and a M13 domain. Without binding of $\mathrm{Ca}^{2+}$ ions to the CaM, the EGFP is in a low fluorescent state (left). CaM can bind up to four $\mathrm{Ca}^{2+}$ ions, due to its $\mathrm{E}-\mathrm{F}$ motifs. Once bound to $\mathrm{Ca}^{2+}$, CaM undergoes a conformation change, which allows it to bind to the M13 domain and in turn effect the fluorophore, leading to an increase in fluorescence (right). Adapted after tian2012imaging

could be measured at the same time thereby providing an internal control, as for example the cold cell could serve as a control for genetically manipulated hot cells, ensuring that the setup and GECI work fine. The occurrences of overlaying cold and hot cells showed a distinctive double peaked response, making it easy to discard them from the dataset.

\subsubsection{Temperature stimulation}

To assess the responses of the arista-neurons to temperature changes, flies were fixed and stimulated with different temperature steps, using a thermo electric generator:

Adult Drosophila were glued to a coverslip ( $24 \mathrm{~mm}$ X 24mm), using Heliobond dental glue (Ivoclar Vivadent, Ellwangen, Germany), which cures under ultraviolet light and is clear, allowing imaging through it. Flies were positioned so that the top of the head and the second antennal segment would touch the coverslip, with dental glue covering the top of the head and the antenna, including the arista. Then, the Heliobond glue would be hardened using a handheld UV lamp (Starlight Pro, mectron, Cologne, Germany). The rest of the body was not immobilized. This preparation would eliminate all movement that would interfere with the imaging of the arista, while still leaving thorax and abdomen free, so as to not block trachea and ensure the survival of the fly for the duration of the experiment. The animal would be angled in such a way that the arista would be very close to the surface of the coverslip (see figure 8). The coverslip with the attached Drosophila was then placed above a thermoelectric generator. This generator makes use of the Peltier effect, allowing to change the temperature by applying a voltage. The temperature generator 
A

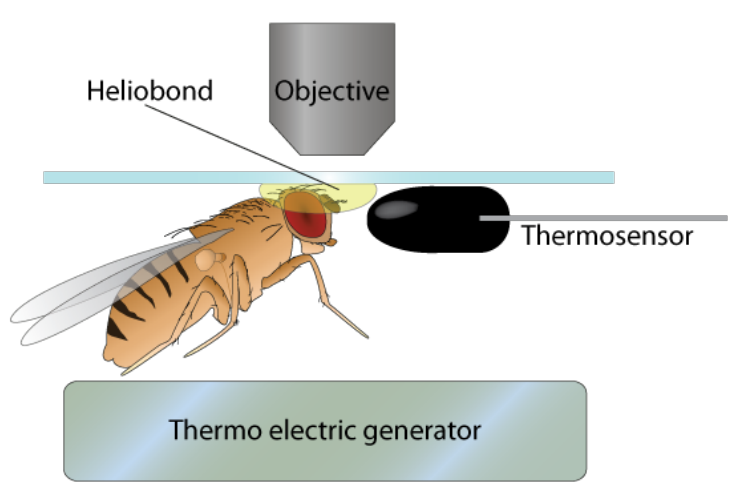

B

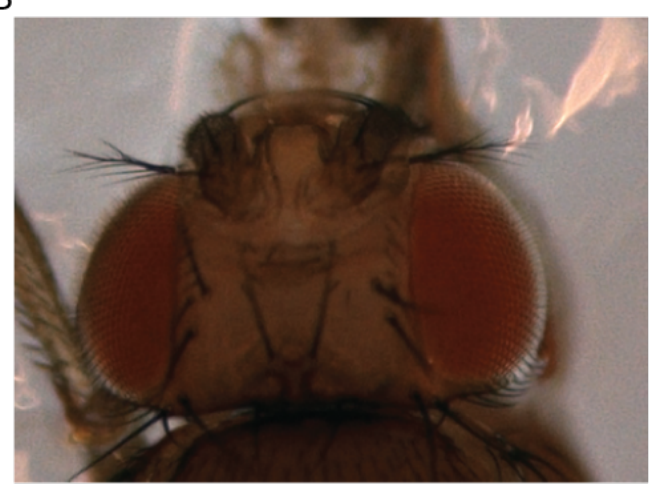

Figure 8: Overview of Drosophila preperation for $\mathrm{Ca}^{2+}$ imaging experiments with temperature stimulation A) Illustration of the preperation for $\mathrm{Ca}^{2+}$ imaging experiments, using temperature stimulation (side view). The fly is glued underneath a glass coverslip, using Heliobond dental glue. Only the top of the head, thorax and the antennae are fixed. The coverslip is placed atop of an thermo electric generator, allowing for temperature stimulation of the fly. A thermosensor is placed in close proximity to the fly, in order to accurately estimate the temperature reaching the animal. (Drosophila illustration altered after Dr. F. Bilz) B) Top-down view of the preperation, during an experiment. To reduce movies size, only a $\mathrm{ROI}$ around the antenna of the animal would be filmed during a recording.

was placed on an aluminum slab (measuring: $150 \mathrm{~mm} \times 170 \mathrm{~mm} \times 10 \mathrm{~mm}$ ) that stabilizes the setup on the microscope table and functions as an additional heat sink. A small 3D printed plastic rim was used to hold the coverslip, with the attached animal, closely above the thermo electric generator. An external temperature sensor (SEMI833ET, B+B ThermoTechnik GmbH, Donaueschingen, Germany) was positioned close to the fly, in order to get an more accurate readout of the temperature that the animal is experiencing during the experiment. The thermo electric generator was connected to a JUMO controller (JUMO dTRON 316 703041/181-400-23/000, JUMO GmbH \& Co. KG, Fulda, Germany), which directly controls the voltage (and with that the temperature) of the generator, and also receives a sensor readout from the thermoelectric generator. The JUMO contoller displays the present temperature and the target temperature of the generator on a front display.

An Arduino micro (arduino.cc) receives input from the JUMO temperature controller, a separate input from the external temperature sensor in proximity of the animal, and receives a timing signal from the camera. Camera and Arduino micro were connected to the same PC. The PC runs a MatLab (The MathWorks, Natick, MA, USA) script/GUI, controlling and monitoring the temperature of the thermo setup. Using a proportional-integral-derivative controller (PID), a temperature, or a succession of different temperature steps can be set for the system. The GUI will display 3 different data traces in this setup: 1. The target 


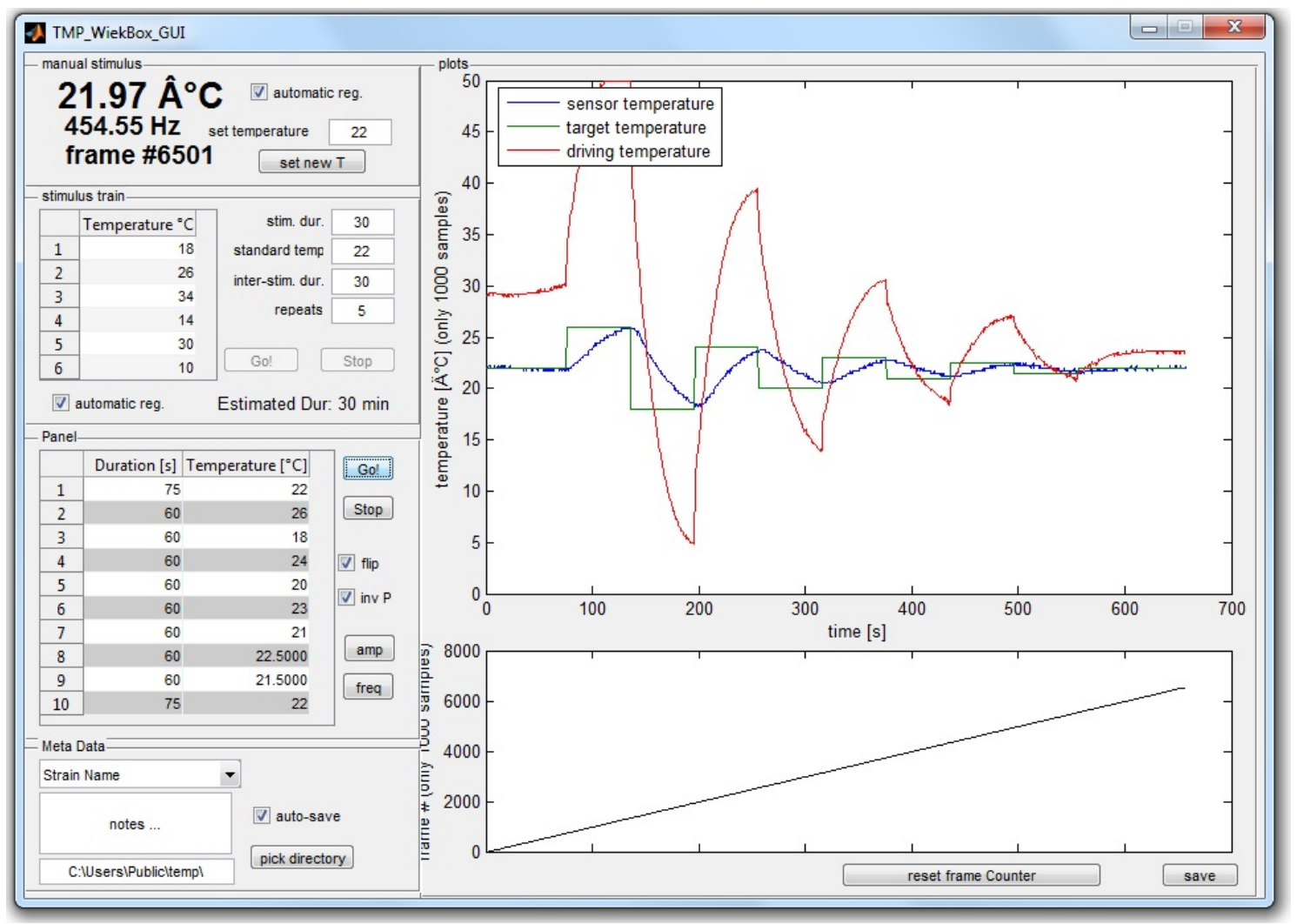

Figure 9: Temperature stimulation control GUI A representative example of the GUI in control of the thermo electric generator during a calcium imaging experiment. Left side show the area in which different temperature steps and their duration can be set an the icons to start the temperature run. Top right shows a temperature curve during an experiment. Blue: sensor temperature; Green: Target temperature; Red: Driving temperature. Note that the step-wise appearance of the curve is a result of the graphical representation showing a reduced number of samples in order to save computational power. Bottom right shows a graphical representation of the number of video frames recorded during the experiment.

temperature (the temperature that is supposed to be applied to the fly) 2 . The sensor temperature (meaning the temperature value recorded by the external sensor close to the Drosophila) 3. The so called driving temperature (the temperature of the thermoelectric element, controlled by the JUMO temperature controller). The PID is supposed to alter the driving temperature, depending on how strong the difference between the sensor temperature and the target temperature is. As temperature is a more inertial type of stimulus, compared to, for example, light or mechanical stimulation, it was not possible to achieve a stimulus that reaches the desired temperature in a very short time window (this is especially true for large temperature differences). Instead, this setup, with the proper settings of the PID, which had to be empirically tested, allows for a "smooth approach" to the target temperature, without much of an over- or undershooting, over the time course of approximately 60 seconds. A more aggressive heating or cooling would 
reach the target temperature sooner, but would also overshoot the desired value (often followed by a oscillation of the sensor temperature around the target value), leading to less precise and less reproducible stimuli.

A

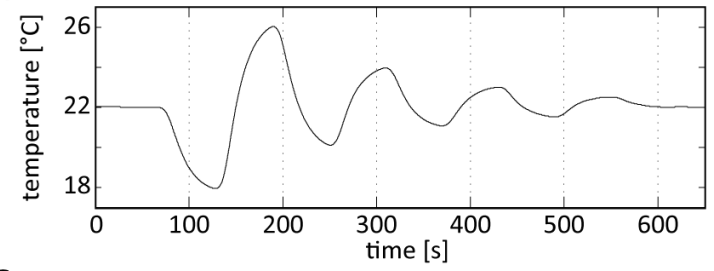

C

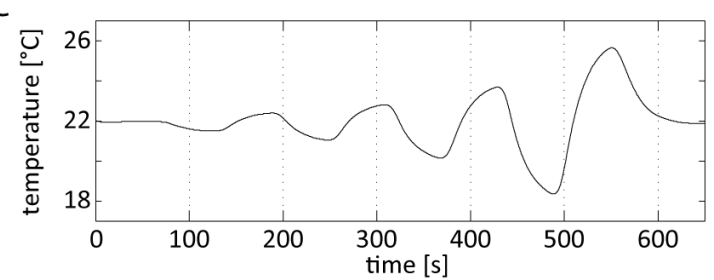

$\mathrm{E}$

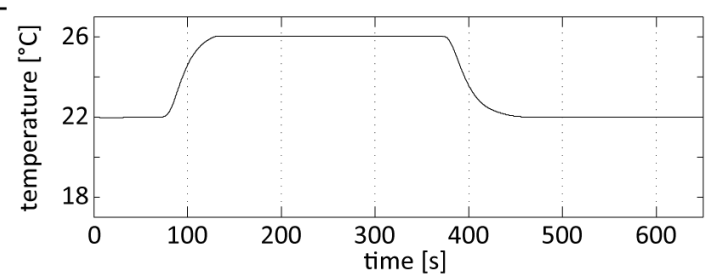

B

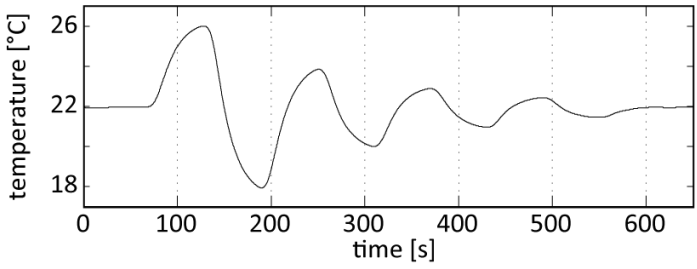

D

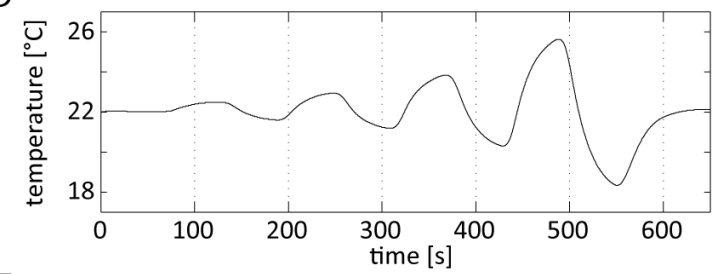

$\mathrm{F}$

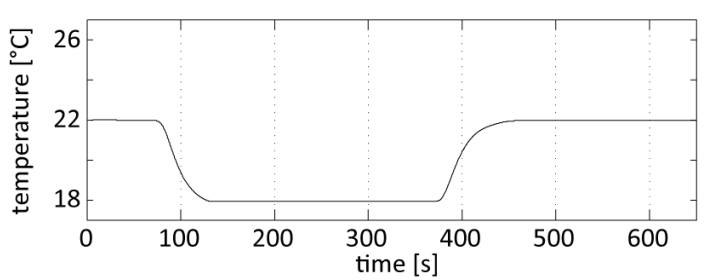

Figure 10: Temperature stimuli during $\mathrm{Ca}^{2+}$ imaging experiments An overview of the different temperature stimuli, one of which was applied during a calcium imaging experiment via a thermo electric generator. Curves show the temperature recorded from a sensor in close proximity to the fly. A)-D) Four different temperature stimuli, used to investigate responses of arista neurons to different relative and absolute temperature steps. A initial waiting period of $75 \mathrm{~s}$ at $22^{\circ} \mathrm{C}$ baseline temperature is followed by alternating steps of temperature increments and decrements (compared to the baseline), either increasing or decreasing in amplitude over the course of the experiment. After 8 Temperature steps, temperature is returned to the baseline temperature of $22^{\circ} \mathrm{C}$. E-F) Temperature protocols applied in order to investigate adaptation over time. After a starting periond of $75 \mathrm{~s}$ at $22^{\circ} \mathrm{C}$ baseline, a single temperature step of either $26^{\circ} \mathrm{C}(\mathrm{E})$ or $18^{\circ} \mathrm{C}(\mathrm{F})$ is applied for 5 minutes, followed by a return to the baseline temperature of $22^{\circ} \mathrm{C}$.

The Matlab script/GUI would furthermore register the frame count of the video that was recorded during the experiment (via the timing input from the camera to the Arduino Mirco). This would later allow to precisely correlate the temperature data with the imaging movie.

Different temperature protocols were used in order to investigate the responses of hotand cold-cells to different relative and absolute changes in temperature. Each protocol consisted of a starting period at a baseline temperature of $22^{\circ} \mathrm{C}$. This starting period was followed by alternating steps of temperature increases and decreases, in either rising or falling amplitude, with a total protocol duration of 650 seconds (75 seconds baseline 
temperature at the beginning, 8 temperature steps of 60 seconds each, and 75 seconds back at base temperature in the end). Alternatively. the initial waiting period would be followed by a 300 second lasting temperature step, followed by a return to baseline (see figure 10). At the end of each recording, the temperature data, along with the corresponding frame/time data would be saved as a .mat file.

\subsubsection{Mechanical stimulation}

To investigate whether mechanical stimulation/deflection of the arista would lead to an activation of the arista-neurons, recordings were performed in a setup similar to the temperature stimulation described in 2.4.1. For this experiment, adult Drosophila (age 2-6 days after eclosion) were again glued to a coverslip using UV hardening Heliobond dental glue, however only the funicle, pedicle and front of the head capsule were covered in glue, to eliminate any movement from the antennal segments while the arista itself remained free. A pulled glass capillary with a hook shaped bend was attached to a piezo actuator (Physik Instrumente, Karlsruhe, Germany) in order to mechanically stimulate the arista. The piezo was controlled by a modular controller (E-501, Physik Instrumente, Karlsruhe, Germany) connected to a SD9 Grass stimulator (MODEL SD9E, Grass Medical Instruments, Quincy, USA).

Once the fly was placed underneath the microscope, the bend tip of the glass capillary was brought into position using a micromanipulator, so that it just touched the arista at the distal part (approximately at $2 / 3$ of the entire arista length). A voltage step, produced by the Grass stimulator was then used to deflect the arista. During the experiment, the temperature was kept at a baseline temperature of $22^{\circ} \mathrm{C}$, as to not elicit any temperature induced neuronal responses.

The arista would be deflected continuously for $60 \mathrm{~s}$. The mechanical stimulation would be followed by a 1 minute pause window and two temperature steps $\left(20\right.$ and $24^{\circ} \mathrm{C}$ ), in order to ensure that cells were alive and responsive during the experiment, and to identify $\mathrm{HC}$ and CC populations.

\subsubsection{Calcium imaging analysis}

Analysis of calcium imaging movies was performed with FIJ (https://fiji.sc). Small instances of image drift along the $\mathrm{X}$ - or $\mathrm{Y}$-axis were corrected for by using the template matching and slice alignment plugin for FIJI (Plugin can be found at: https://sites . google.com/site/qingzongtseng/template-matching-ij-plugin). To correct for photobleaching during the course of the recording, a region of interest (ROI) was drawn in an area of the movie without abrupt changes in fluorescence and FIJI's integrated bleach correction function was employed, using an exponential fit. 
To assess the relative change in fluorescence, the measure $\Delta \mathrm{F} / \mathrm{F}_{0}$ (change of fluorescence divided by baseline fluorescence) was used. The calculations for this part of the analysis were performed with FIJI's "Image Calculator" function.

The first step to this end was to calculate the baseline fluorescence $\left(f_{0}\right)$ by generating a picture of the average intensity of the first 20 frames of a movie, using FIJ's "Z-Project" function. By subtracting this baseline image from each of the 6500 frames of a movie, the change in fluorescence was obtained $(\Delta \mathrm{F})$. Finally, each of these 6500 difference images were then divided by the baseline fluorescence $\left(F_{0}\right)$.

By drawing a ROI around the cell body of an arista neuron in the obtained $\Delta F / F_{0}$ movie, the change in fluorescence of the corresponding cell could be obtained (using the function "Plot Z-Axis Profile"). The ROI was drawn at the cell bodies of the neurons, as they would give of a strong and reliable signal, viewable even thorugh the cuticule, whereas the dendrites of the cell (due to being significantly thinner) would often not be visible in the experiment.

\subsubsection{Analysis of temperature stimulated $\mathrm{Ca}^{2+}$ imaging}

Calcium traces and the corresponding temperature curves were correlated using a Python script, extracting the data from the $\Delta F / F_{0}$ file (see section 2.4.3) and the temperature file saved after each experiment and aligning them via the framenumber (as this information is saved in both data files). The resulting file, containing both the timing information as well as the values for the sensor temperature and the $\mathrm{Ca}^{2+}$ trace, was then used in the further analysis, which was conducted using custom MatLab scripts. The first step in this analysis was a final drift correction, to eliminate remaining artifacts from bleaching and movement during the recording (which were not eliminated during the steps described in 2.4.3). The correction was done fit a cubic polynomial curve to the $\Delta F / F_{0}$ curve and subtracting this fit from the curve. A cubic polynomial was chosen, as it most closely matched the nonlinear curve drift, resulting from movement and photobleaching. Imaging data would be grouped, with respect to fly strain and stimulus conditions. Median and mean $\mathrm{HC}$ and CC responses and the corresponding SEM (mean) or confidence intervals (median) for each strain at each stimulus condition were calculated.

As the different stimulus conditions, shown in Figure $10 \mathrm{~A}-\mathrm{D}$, generate a set of absolute and relative temperature changes, peak responses to temperature changes were compared by establishing a 10 frame timewindow around the maximum of a temperature step response and comparing calcium response amplitude in that window. To compare the responses to relative changes, the $\Delta \mathrm{F} / \mathrm{F}_{\mathrm{O}}$ response for different temperature steps with the same relative step size (for example a step from 22 to $26^{\circ} \mathrm{C}$ and from 18 to $22^{\circ} \mathrm{C}$ would both constitute a relative change of $+4^{\circ} \mathrm{C}$ ), the median fluorescence change was plotted in regards to the relative temperature step amplitude. Median and mean $\Delta F / F_{0}$ 

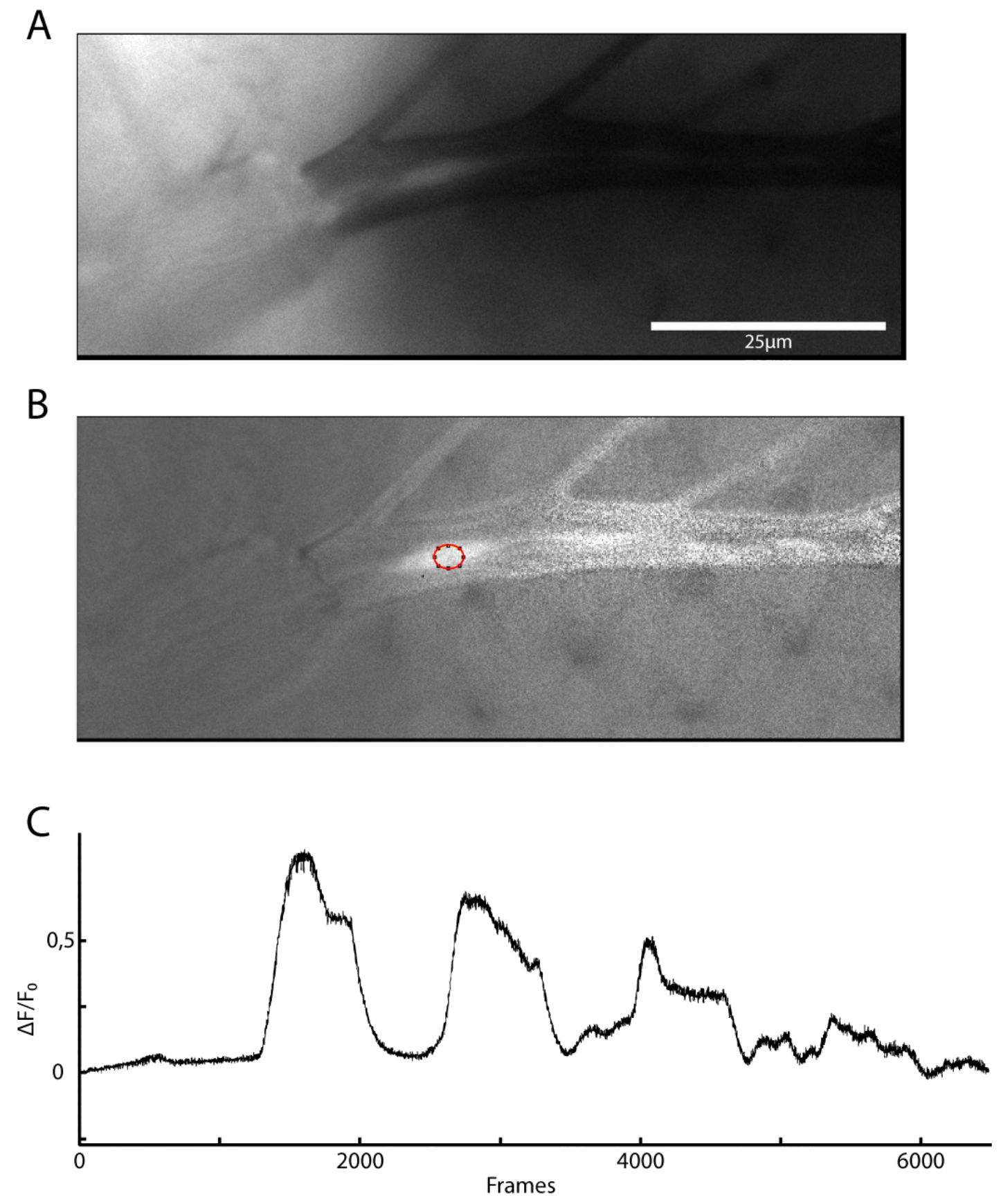

Figure 11: Example of calcium imaging analysis A) Raw movie of the arista of an adult Drosophila with neurons expressing the GECl GCaMP6m B) $\Delta F / F_{0}$ result of the image shown in A), after bleach correction and drift correction. The high brightness in the difference image implies an increase in neuronal activity / intracellular $\mathrm{Ca}^{2+}$ concentration. A ROI (red) is drawn on one of the responding cells. C) Change in luminescence of the cell marked with a ROI in $\mathrm{B}$ ) over the timecourse of the experiment.

values for all relative temperature step amplitudes would be calculated and the $\Delta F / F_{0}$ value plotted both as boxplot for each individual temperature step and as a function of the relative temperature step size. In order to gain a estimate of statistically significant 
differences in the maximum responses, the gain of the $\Delta \mathrm{F} / \mathrm{F}_{0}$ signal per ${ }^{\circ} \mathrm{C}$ change was calculated for the biggest relative temperature changes of each stimulus condition (for both increments and decrements). To better visualize possible effects of different mutations on relative step response amplitudes in a quick overview, a linear fit was fitted to the relative response curves and the gain of this fit would be calculated. Furthermore, as the temperature stimulation would not produce an instant jump to each target temperature, but a change over time, the $\Delta \mathrm{F} / \mathrm{F}_{\mathrm{O}}$ would also be plotted against the temperature change over time $\left({ }^{\circ} \mathrm{C}\right.$ change / s).

\subsubsection{Analysis of mechanically stimulated $\mathrm{Ca}^{2+}$ imaging}

In the case of mechanical stimulation experiments, $\Delta \mathrm{F} / \mathrm{F}_{0}$ traces of the recorded movies were obtained in FIJ and the resulting imaging traces and temperature data were correlated with the same python script described in 2.4.3.1 The time and duration of the mechanical stimulation was determined directly from the video frame numbers, as the movement of the glass capillary was clearly visible. Mean $\Delta F / F_{0}$ response and SEM to the stimulation was calculated and plotted using Matlab.

\subsection{Temperature preference behaviour}

\subsubsection{Recording of temperature preference behaviour}

In order to assess the preferred and tolerated temperatures of adult Drosophila, their locomotion behaviour on a temperature gradient was recorded. This experimental setup was first established in Giraldo Sanchez 2018; Giraldo et al. 2019. The behavioural experiments were conducted under identical conditions, following strictly the aforementioned methods.

The basis for this gradient arena setup consisted of an aluminum slab, in which 5 lanes (measuring $50 \mathrm{~mm} \times 3 \mathrm{~mm} \times 3 \mathrm{~mm}$ ) were cut. Before the start of an experiment, a brass cylinder containing salt water and frozen in $\mathrm{a}-80^{\circ} \mathrm{C}$ freezer, was placed on one side of the aluminum block, while a set of 4 soldering irons were inserted into the block on the opposing side.

In order to monitor the temperature of the arena, 30 temperature sensors (SEMI833ET, $\mathrm{B}+\mathrm{B}$ Thermo-Technik GmbH, Donaueschingen, Germany) were built into the aluminum block and measured the temperature with a frequency of $10 \mathrm{~Hz}$. A multiplexer, controlled by an Arduino micro (arduino.cc) was used to obtain the sensor data and forward it to a PC, running a MatLab script/GUI. In this script, the user can define a minimum and maximum temperature for the "hot side" of the arena. Once the sensors report a temperature 
lower than the set minimum, the soldering irons would turn on, heating up that end of the aluminum block. If the set maximum temperature was reached, the irons would be automatically shut off. The temperature of the arena could furthermore be live monitored before and during the experiment in the MatLab GUI.

This combination of heating on one side, while cooling down the opposing side, generates a stable temperature gradient, reaching from approximately $14^{\circ}$ on the cold end to $30^{\circ} \mathrm{C}$ on the hot end, lasting well beyond the duration of an experiment. However, since the frozen salt water begins to thaw eventually, the brass cylinder was exchanged after every experiment.

Flies were anesthetized on ice and, once a stable gradient was reached, put into the lanes of the arena, on the hot end. Only female virgin Drosophila, with an age between 2 - 6 days were used in the experiment, as the larger females are easier to locate during tracing, and the use of virgins in the experiment would exclude potential influence of oviposition site search towards the preferred and tolerated temperatures (Dillon et al. 2009). The tracks were covered using a translucent Plexiglas slide, to prevent flies from escaping. The slide was covered

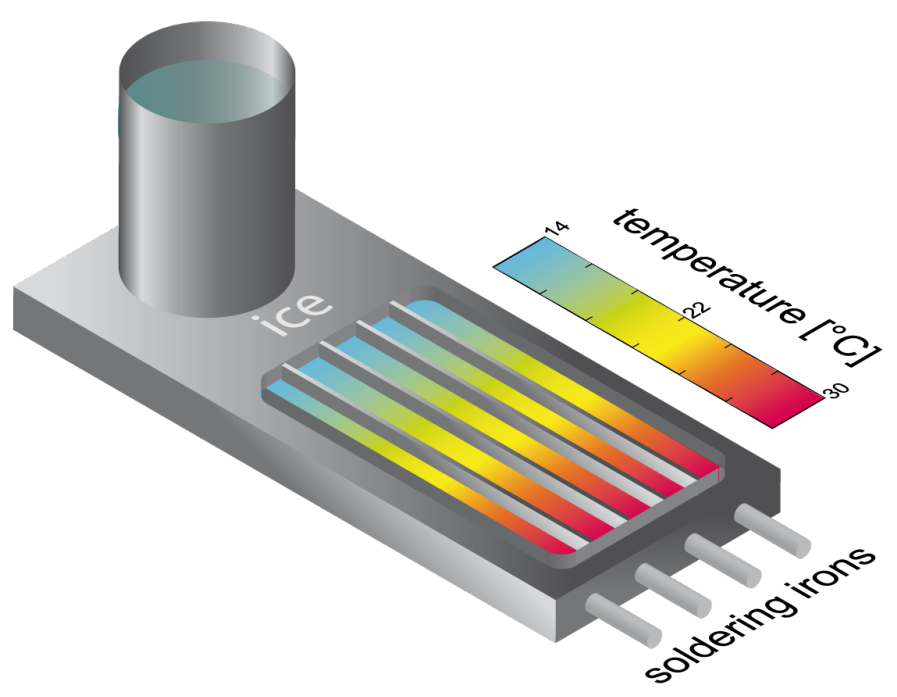

Figure 12: Illustration of temperature preference behaviour arena Illustration shows an overview of the arena used to assess temperature preference and tolerance behaviour of Drosophila. Five lanes were cut into an aluminum slab. A temperature gradient was generated by cooling on side of the aluminum arena with a brass cylinder, filled with frozen salt water (frozen at $-80^{\circ} \mathrm{C}$ ), while the other side was heated with a set of four soldering irons, inserted into slots in the slab. Soldering irons would dynamically be turned on and off to keep a stable temperature range. Each lane of the arena would be filled with a single fly, which, after a short acclimatization period, would be filmed for 5 minutes, while it could move freely within the gradient. Movies were recorded with a framerate of $50 \mathrm{fps}$.

Illustration altered after Dr. D. Giraldo. and Dr. B. R. H. Geurten in Sigmacote (Sigma-Aldrich) to discourage flies from crawling on it.

After all animals were placed into the tracks, they were given 10-12 minutes to recover from cold anesthesia, before a recording was started. During an experiment, flies were recorded from the top, using a GigE camera (Teledyne DALSA,), at 50fps, for 5 minutes. The setup was illuminated using a set of infrared LEDs (940nm \pm 25 ; Bausatz Infrarot- 
Scheinwerfer, Pollin Electronic GmbH, Pförring, Germany), as the flies cannot detect infrared light, thus eliminating interference from visually guided behaviours, such as positive phototaxis.

\subsubsection{Analysis of temperature preference behaviour}
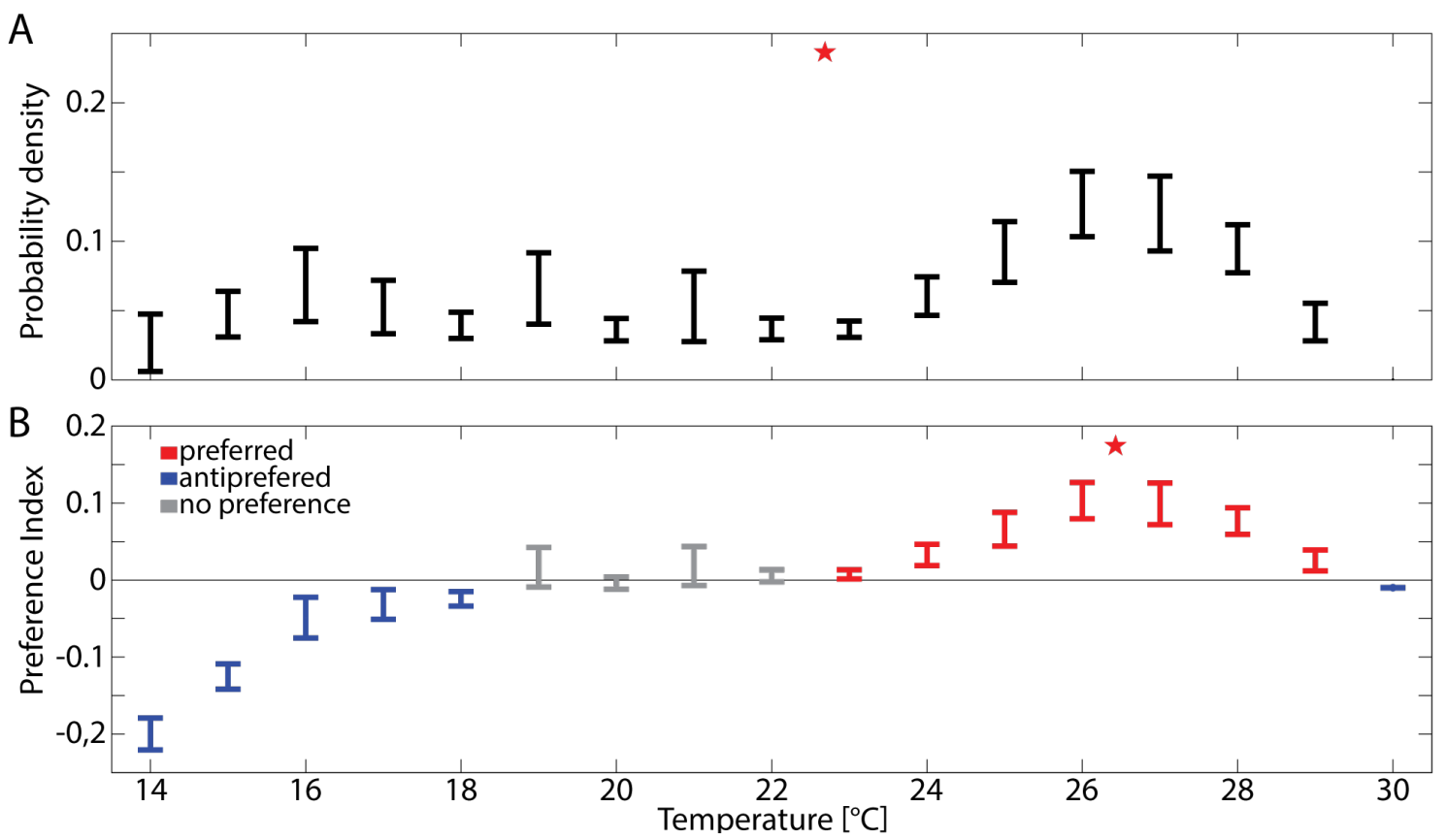

Figure 13: Temperature preference correction via IGLOO A) Histogram of an example fly strains distribution in the temperature gradient locomotion experiment before correction with IGLOO. Red star denotes the mean preferred temperature B) Histogram of the same strain as in A), after cold bias correction via IGLOO, in which a simulated distribution is subtracted from the uncorrected dataset. Temperatures at which the probability density is positive would be regarded as preferred, temperatures with negative probability density as avoided or antipreferred. Should the $95 \%$ confidence interval (error bars) overlap with the 0 axis, the temperature would be regarded as neither avoided nor preferred.

In order to obtain the two-dimensional trajectories of flies moving inside the lanes, movies were analyzed using ivTrace (Jens P. Lindemann, Bielefeld University). The obtained trajectories were then correlated with the temperature data obtained during the experiment, in order to obtain exact information about the temperature that the fly was exposed to over time. To obtain a measure of the preferred and avoided temperature for different Drosophila strains, the probability density of position over the temperature gradient as well as the mean preferred temperature was calculated.

However, as Drosophila are ectothermic animals, a locomotion experiment in a temperature gradient can lead to a bias towards cold temperatures, as the animals metabolism and activity will slow down under certain temperatures, leading to an effect referred to as cold-trapping or cold-sleep. In order to correct for this, the locomotion null model 
IGLOO (Giraldo et al. 2019) was used. This model provides a simulated distribution without any temperature preference, only determined by the effect of the temperature on the animals locomotion behaviour. The simulated distribution can be subtracted from the experimentally obtained distribution for each fly strain. This would result in positive and negative values (see figure 13), which are referred to as preference index (PI). Temperatures at which the preference index was negative were counted as avoided, whereas temperatures with positive preference index would be defined as preferred. Should the $95 \%$ confidence interval of the median value overlap with 0 , the temperature is regarded as neither avoided nor preferred.

\subsection{Microscopy}

\subsubsection{Image Acquisition}

Microscopic pictures of the arista, for the purpose of assessing anatomy and expression patterns, were obtained using a Leica TCS SP8 confocal laser scanning microscope (Leica microsystems, Wetzlar, Germany). Overview images of the head and antenna/arista were obtained using either a 10X (Leica HCX PL FLUOTAR 10X/0.30) or a 20X(Leica HC PL APO 20X/0.75 IMM) objective. Closeup images of the cells in the arista were obtained with a 63X(Leica HC PL APO 63X/1.20 W) immersion objective. Obtained images and 3D stacks were analyzed using FIJI.

\subsubsection{Imaging of Gal4 driven expression patterns}

In order to assess expression of genetically encoded fluorophores, such as GFP, whole Drosophila heads were imaged. Adult flies (age 2-6 days after eclosion) were collected, put under $\mathrm{CO}_{2}$ anesthesia and decapitated. Heads were mounted facing upwards on glass depression slides using a small drop of glue, to prevent movement under the microscope. Heads were immersed in PBS with $1 \%$ TritonX and the slide was covered using a coverslip. Unless otherwise noted, heads were imaged directly after mounting, as the GFP fluorescence would decay within a few hours after the decapitation of the animal.

\subsection{Statistical analysis}

Unless otherwise noted, all plots and the corresponding statistical analysis were performed in Matlab or Python. Statistical significance was tested using Fishers exact permutation test, in order to evaluate the differences in median of the respective tested datasets. The obtained $p$-values were always corrected via Benjamini-Hochberg procedure for false detection rate (Benjamini and Hochberg 1995), implemented in Matlab by 
D. Groppe and colleagues (Groppe, Urbach, and Kutas 2011).

Statistical significances in figures are denoted in regards to $p$-values as: ${ }^{*}=p<0.05 ;{ }^{* *}$ $=\mathrm{p}<0.01 ;{ }^{* *}=\mathrm{p}<0.001 ;$ NS: not significant.

When data is presensted as boxplots, the red lines denote median values, box indicates upper and lower quartile range (50\% of the data set). When no outliers are present, the whiskers indicate $100 \%$ of the dataset, if outliers are present, the whiskers indicate 1.5 interquartile distance. Black crosses denote outliers. 


\section{Chapter 3}

\section{Results}

\section{1 nompC is expressed in the hot cells of the arista}

Research has well established that the arista of Drosophila melanogaster houses a population 6 neurons, which have been found to be temperature sensitive, and, depending on their response behaviour towards temperature, are named hot-cells ( $\mathrm{HC}$ ) and coldcells (CC) (Foelix, Stocker, and Steinbrecht 1989; Gallio et al. 2011; Ni, Bronk, et al. 2013). As described in 1.2, preceding studies in our lab found hints that the mechanosensory TRP channel NOMPC might be found in these arista neurons, as expressing GFP under nompC-Gal4 control resulted in an observable fluorescent signal in the base of the arista. A preceding experiment, performed by A. Adden during the work for her Master thesis, indicated that nompC expression might be confined to the $\mathrm{HC}$ population of arista neurons. To confirm the localization of NOMPC in the arista, I studied the expression of membrane associated UAS-mcd8-GFP via different Gal4 lines.

Driving expression of GFP via nompC-Gal4 results in an observable signal from 3 cells (Figure $14 \mathrm{~A}$ ), meaning that nompC is apparently not expressed in all neurons of the arista. In order to investigate whether NOMPC localized specifically in either the HC or CC subsets of neurons in the arista, I used specific driver lines for each of the cell groups: HC-Gal4 (Gallio et al. 2011) only shows expression in the HC population (Figure $14 \mathrm{~B}$ ), whereas NP4486-Gal4 (brv1, from hereon referred to as CC-Gal4) expression in the arista is confined to the CC population (Figure 14 D) (Gallio et al. 2011). By using either HC- or CC-Gal4 together with the nompC-Gal4 to drive the GFP expression, this overlap experiment can give clues to the cell group in which NOMPC localizes. A similar approach to cellular localization was performed in 2013 (Ni, Bronk, et al. 2013) to identify the cell subset expressing the gustatory receptor GR28B(D). Initial experiments were performed by A. Adden during the work on her Master thesis.

When driving GFP expression with both HC-Gal4 and nompC-Gal4, I could only observe up to 3 fluorescent cells (Figure 14 C). Sometimes cells were located so closely to each other, 
that even with a confocal microscope, it was impossible to disentangle their anatomy, so that I only could count two cells with certainty. . When employing both CC-Gal4 and nompC-Gal4 on the other hand, up to six cells would be labelled (Figure $14 \mathrm{E}$ ). I can therefore conclude, that the expression of nompC in the neurons of the arista is specific to the $\mathrm{HC}$ population of thermosensitive neurons. 
A

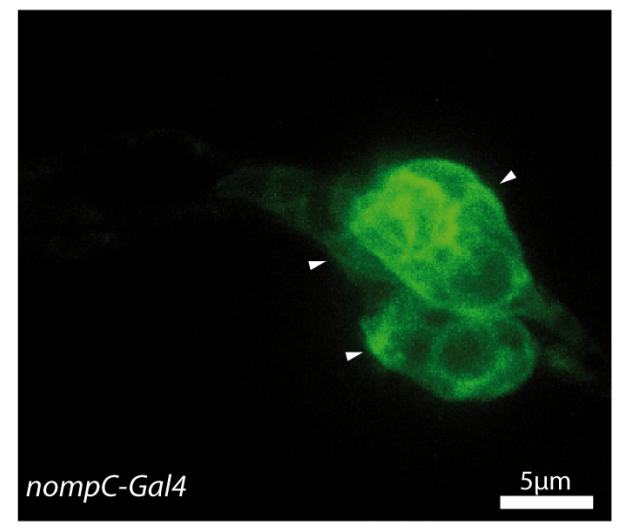

C

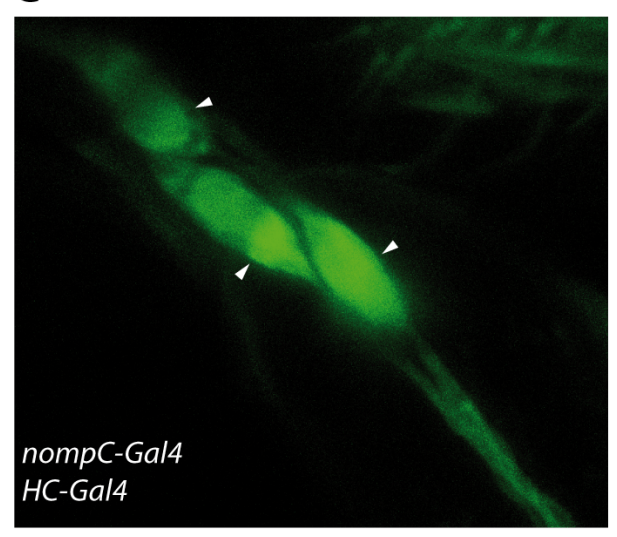

$\mathrm{E}$

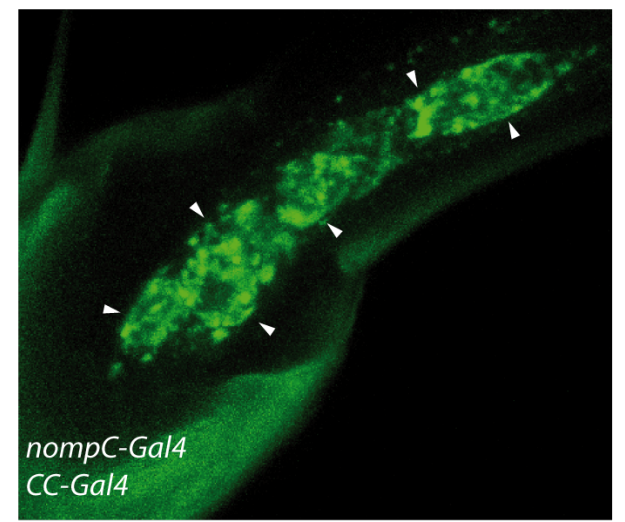

B

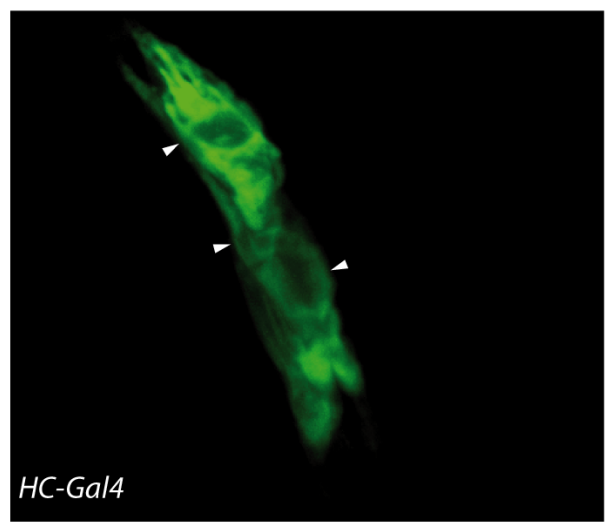

D

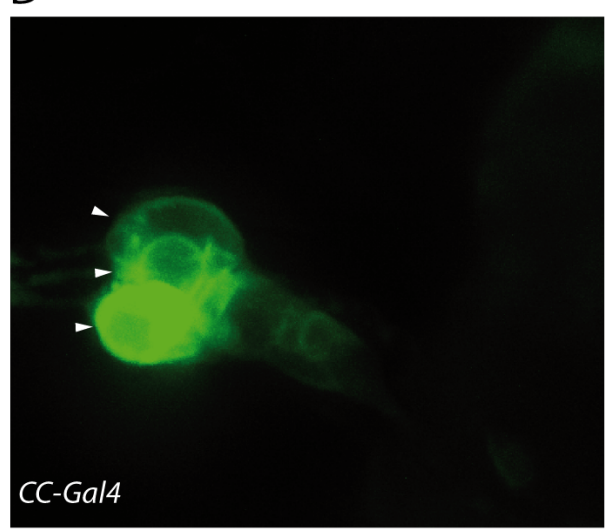

$\mathrm{F}$

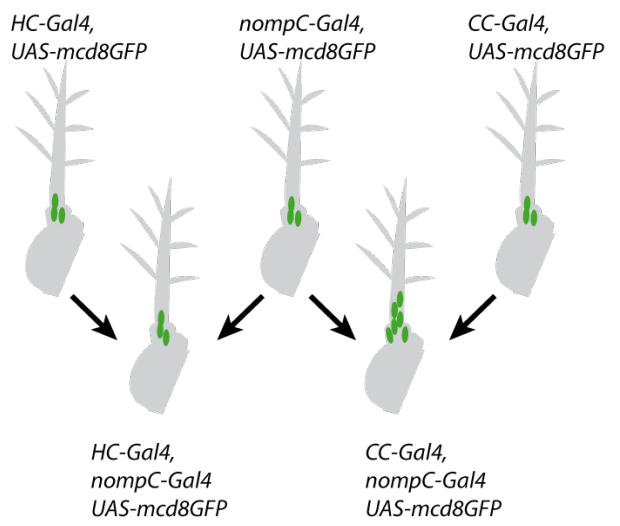

Figure 14: Cellular expression pattern of nompC Expression of membrane associated mcd8-GFP under UAS control in the neurons of the Drosophila arista. Maximum projections of confocal image stacks. A) nompC-Gal4 driving UAS-mcd8-GFP expression labels only 3 of the arista neurons. B) HC-Gal4 driver, labeling the $3 \mathrm{HCs}$ of arista neurons C) Using both nompC-Gal4 and HC-Gal4 to drive GFP expression labels 3 cells, suggesting an overlapping expression pattern. D) CC-Gal4 driver, labeling the 3 CCs of arista neurons E) Using both nompC-Gal4 and CC-Gal4 to drive GFP expression. This combination of Gal4 drivers labels up to 6 neurons in the arista, suggesting no overlap in the expression pattern. This suggests that nompC expression is limited to the HCs of arista neurons. F) Schematic illustration of the overlapping expression experiment. Each of the employed Gal4 driver lines labels three cells in the arista. Only the combination of CC-Gal4 and nompC-Gal4 labelled all six arista neurons with the GFP under UAS control, suggesting an overlap of HC-Gal4 and nompC-Gal4 expression. 


\subsection{Hot and cold cells show relative calcium responses to temperature stimuli}

When work on the study presented here began, the response properties of hot and cold cells were unknown, except of the fact that cold cells respond with deplorisations to temperature decreases and hot cells depolarise when stimulated with temperature increases (Gallio et al. 2011). Especially whether the sensors are operating in a relative or absolute response manner was unknown. It was therefore paramount to establish the response properties of the temperature sensitive cells before a more detailed analysis of nompC role could be undertaken. Luckily, a very recent publication confirmed the findings presented in the following chapter (Budelli et al. 2019).

Flies were exposed to a regime of different temperature steps during $\mathrm{Ca}^{2+}$ imaging experiments, as described in 2.4.1. Panneuronal expression of the genetically encoded calcium indicator (GECI) GCaMP6m allowed for the assessment of $\mathrm{Ca}^{2+}$ responses in both $\mathrm{HC}$ and CC populations simultaneously. This allowed us to monitor the quality of every trial based on the wildtype signals.

The first temperature stimuli presented consisted of alternating steps of temperature increases and decreases, with either increasing or decreasing amplitude (as described in Figure $10 \mathrm{~A}-\mathrm{D})$. Both $\mathrm{HC}$ and $\mathrm{CC}$ populations showed a distinctive response pattern to both temperature increments and decrements (Figure 15): HCs showed an increase in fluorescence (implying an excitatory response of the neurons) in response to temperature increments. Temperature decrements resulted in a decrease of overall fluorescence, dropping below the baseline value of $\Delta F / F_{0}=0$ (implying a hyperpolarization). In contrast CCs exhibit the exact opposite response behaviour, showing excitation towards temperature decrements and inhibition during temperature increments. This mechanism of both excitatory and inhibitory response patterns to temperature in either direction in both cell populations implies that, contrary to what the given names of the cell populations might suggest, both HCs and CCs respond to both increases and decreases in temperature.

Flies of both wildtype control strains, in either the Cantons or $w^{1118}$ background showed robust temperature evoked $\mathrm{Ca}^{2+}$ responses, in which the amplitude of the response is clearly correlated to the amplitude of the temperature change (Figure 15). No obvious difference in response amplitude can be observed between the two strains with different genetic background, implying that the $\mathrm{Ca}^{2+}$ response amplitude provides a reliable measurement of the neuronal activity that can be compared between different strains. 


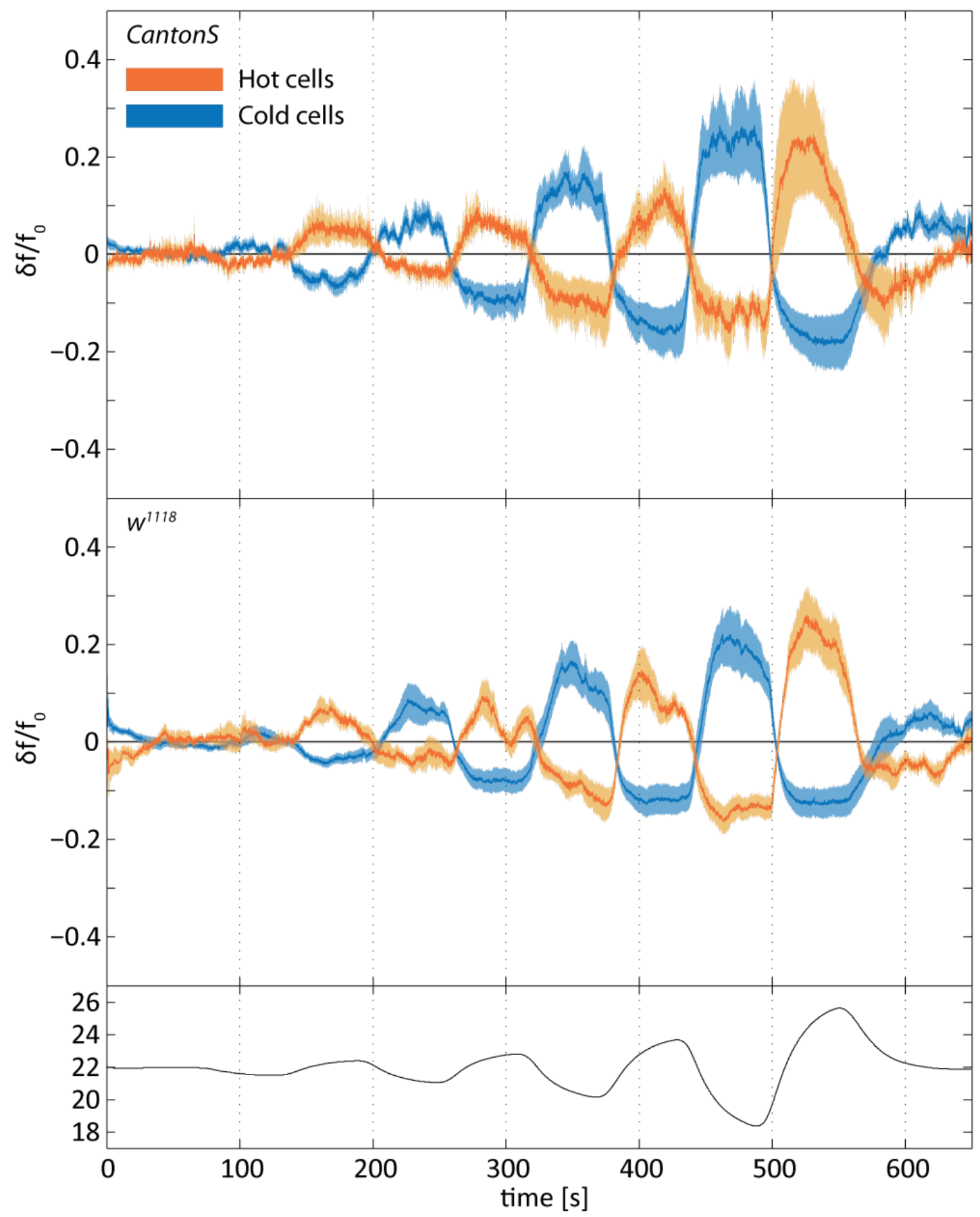

Figure 15: Temperature evoked $\mathrm{Ca}^{2+}$ responses of wildtype flies Calcium responses of CantonS and $w^{1118}$ control flies to alternating heat and cold steps. Top: $\Delta F / F_{0}$ response of CantonS flies to the temperature stimuli shown in the bottom plot. $\mathrm{HC}$ responses in orange, $\mathrm{CC}$ responses in blue. Solid line denotes mean response of the respective cell type, shaded area represents SEM. Middle: $\Delta F / F_{0}$ response of $w^{1118}$ flies Bottom: Temperature stimulus.

HCs show an increase in fluorescence in response to temperature increases and a reduction of fluorescence in response to temperature decreases. CCs show the opposite response behaviour.

n: Cantons HCs, CCs, w ${ }^{1118}$ HCs, CCs. 
Interestingly, stimulation with the same absolute temperature did not generate the same amplitude of $\mathrm{Ca}^{2+}$ responses in different stimulus protocols: As can be seen for example in figure 16 , both the stimulus conditions in A) and B) use the same absolute temperature steps, but in a different order. The temperature increase to $26^{\circ} \mathrm{C}$ elicits a excitatory response of $\mathrm{HCs}$ in both conditions, however, the response amplitude in $\mathrm{B}$ ) is visibly higher than in $\mathrm{A}$ ). In the stimulus shown in $\mathrm{B}$ ), the temperature changes from 18 to $26^{\circ} \mathrm{C}$ (a relative change of $8^{\circ} \mathrm{C}$ ), whereas in $\mathrm{A}$ ), the $26^{\circ} \mathrm{C}$ step is preceded by $22^{\circ} \mathrm{C}$ (a relative change of $4^{\circ} \mathrm{C}$ ). Similarly, when comparing the first and second temperature increase step seen in Figure $16 \mathrm{~A}$, the second temperature increase (towards $24^{\circ} \mathrm{C}$ ) encompasses a relatvie change of $6^{\circ} \mathrm{C}$, end elicits a higher response amplitude in $\mathrm{HCs}$ than the first increment, which only encompasses a $4^{\circ} \mathrm{C}$ change, even though it reaches a higher absolute temperature (of $26^{\circ} \mathrm{C}$ ). This shows that the temperature evoked $\mathrm{Ca}^{2+}$ response amplitude is to a great extend determined by the relative, rather than absolute change in temperature. A complete overview of $\Delta F / F_{0}$ response curves for all employed stimulus paradigms and fly strains can be found in the Appendix, sorted by stimulus protocols in figures A1 - A1, and sorted by fly strain in figures A5 - A11

This response behaviour can be further illustrated when comparing the $\mathrm{Ca}^{2+}$ responses that are triggered by temperature steps that result in the same relative change in temperature albeit at different absolute temperatures (Figure 17).

In the employed temperature step stimulus paradigms shown in figure $10 \mathrm{~A}-\mathrm{D}$, a relative temperature increase of $4^{\circ} \mathrm{C}$ occurs in three different absolute temperature conditions (at 18 to $22^{\circ} \mathrm{C}$, at 20 to $24^{\circ} \mathrm{C}$ and at 22 to $26^{\circ} \mathrm{C}$ ). When comparing for example the responses of HCs of the $w^{1118}$ control strain to these 3 different step conditions, no significant difference between the three stimulus conditions can be observed (as shown be the overlapping confidence intervals). Furthermore, no clear trend with increasing absolute temperature values can be observed, neither in the comparison of relative, peak to peak change of $\Delta F / F_{0}$ values (Figure $17 \mathrm{~A}$ ), nor in the absolute $\Delta F / F_{0}$ responses (Figure $17 \mathrm{~B}$ ). Conversely, when comparing temperature steps that end with the same absolute temperature, but encompass different relative changes, this response behaviour was further demonstrated (Figure 18. Comparing for example the $22.5^{\circ} \mathrm{C}$ temperature step, which occurs with 3 different temperature changes in the stimulus protocols, revealed a clear trend: Higher relative changes resulted in higher response amplitudes. A significant difference could be observed between the smallest $\left(0.5^{\circ} \mathrm{C}\right)$ and the largest $\left(1.5^{\circ} \mathrm{C}\right)$ relative temperature change (Figure $18 \mathrm{~A}$ ).. This further corooborates my hypothesis that the response amplitude of the arista neurons are determined by relative changes in temperature.

To analyse the effect of relative temperature changes on the $\Delta \mathrm{F} / \mathrm{F}_{0}$ signal of the neurons, the mean responses were plotted against the relative temperature step amplitude (Figure 19). As shown above, HCs react to temperature increments with an increase in flu- 

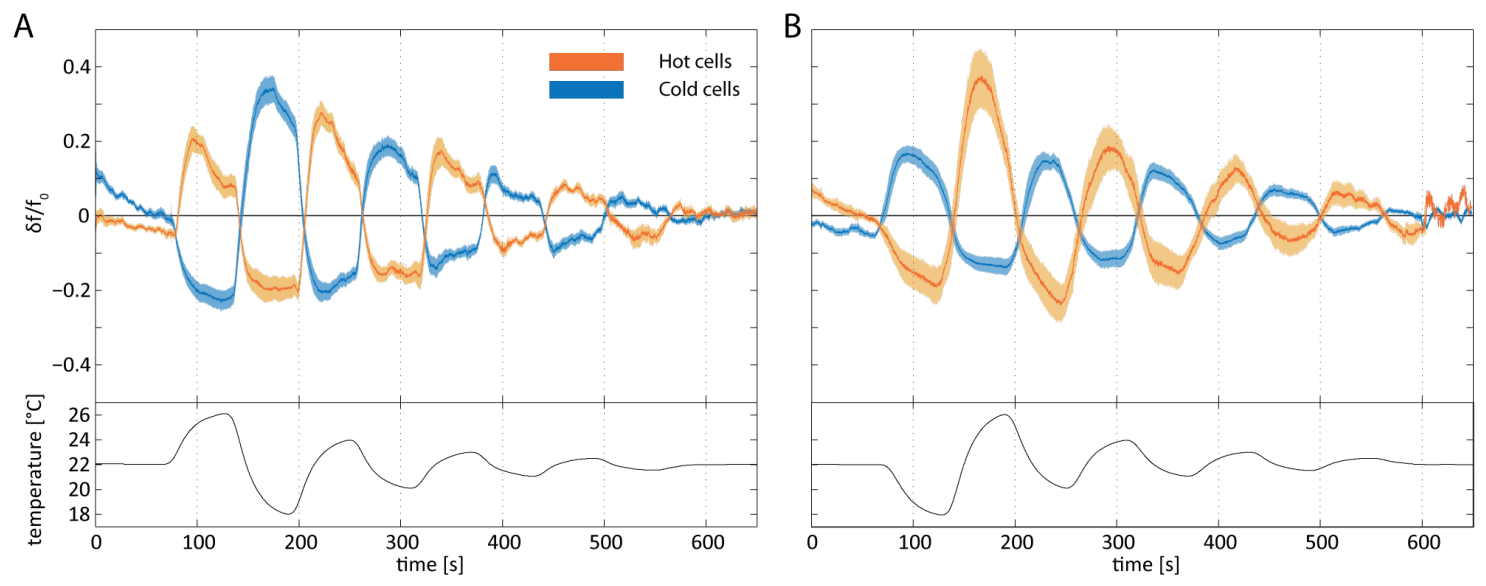

Figure 16: Comparison of $\mathrm{Ca}^{2+}$ responses to different temperature stimulus protocols The responses of both HCs and CCs in $w^{1118}$ control flies to a different sequence of temperature stimuli is shown. $\mathrm{HC}$ responses shown in orange, $\mathrm{CC}$ responses in blue. Solid line denotes mean response, shaded area is SEM. A) Temperature stimuli are decreasing in amplitude with each step. The first step is a temperature increase to $26^{\circ} \mathrm{C}$. $\mathrm{HC}$ response shows a higher amplitude to the second temperature increase ( to $24^{\circ} \mathrm{C}$ ) than to the first step (increase to $26^{\circ} \mathrm{C}$ ). As the first temperature increase encompasses a relative temperature change of $4^{\circ} \mathrm{C}$, while the second temperature increase a relative change of $6^{\circ} \mathrm{C}$, the $\mathrm{HC}$ amplitude seems to be strongly affected by the relative change in temperature. $\mathrm{n}: 10$ HCs, 13 CCs. B) Temperature stimuli are decreasing in amplitude with each step. The first step is a temperature decrease towards $18^{\circ} \mathrm{C}$. $\mathrm{HC}$ response amplitude to the $26^{\circ} \mathrm{C}$ step is higher than to the same absolute temperature in $\mathrm{A}$, as the $26^{\circ} \mathrm{C}$ step is preceded by a $18^{\circ} \mathrm{C}$ temperature step in this paradigm, but preceded by the $22^{\circ} \mathrm{C}$ baseline temperature in A). This further implies the relative temperature change as the determining factor of $\mathrm{Ca}^{2+}$ response amplitude. n: $5 \mathrm{HCs}, 10 \mathrm{CCs}$.

orescence and to temperature decrements with a reduction in fluorescence, while CCs show an opposite response pattern. The response amplitude clearly rises with higher relative temperature step amplitudes for both $\mathrm{HC}$ and CC populations. CantonS and $w^{1118}$ show a strong overlap in their respective mean response curves. Cantons flies reach the strongest increase in $\Delta \mathrm{F} / \mathrm{F}_{\mathrm{O}}$ in $\mathrm{HCs}$ at a temperature step of $+6^{\circ} \mathrm{C}$ (the second largest temperature increase in this experimental condition), with a $\Delta F / F_{0}$ mean response of 0.3 , while the strongest excitatory $\mathrm{CC}$ response can be observed at the $-8^{\circ} \mathrm{C}$ step with a mean response of 0.43 (Figure 19. Inhibitory responses reached their maximum for $\mathrm{HCs}$ at the $-8^{\circ} \mathrm{C}$ step, with a mean $\Delta \mathrm{F} / \mathrm{F}_{0}$ response of -0.38 , and for $\mathrm{CCs}$ at $+6^{\circ} \mathrm{C}$ with a mean value of -0.42 . The fact that the increase in response to stronger temperature changes appears to rise less strongly at higher temperatures might imply a saturation of the response, fitting nicely into the typically sigmoidal shaped response curve found in many receptor neurons (see for example Kandel et al. 2000; Moyes and Schulte 2008). A similar response pattern can be observed in $w^{1118}$ flies, in which the largest temperature increase $\left(+8^{\circ} \mathrm{C}\right)$ also elicits the strongest excitatory response in the HCs. Inhibitory responses peak at rel- 

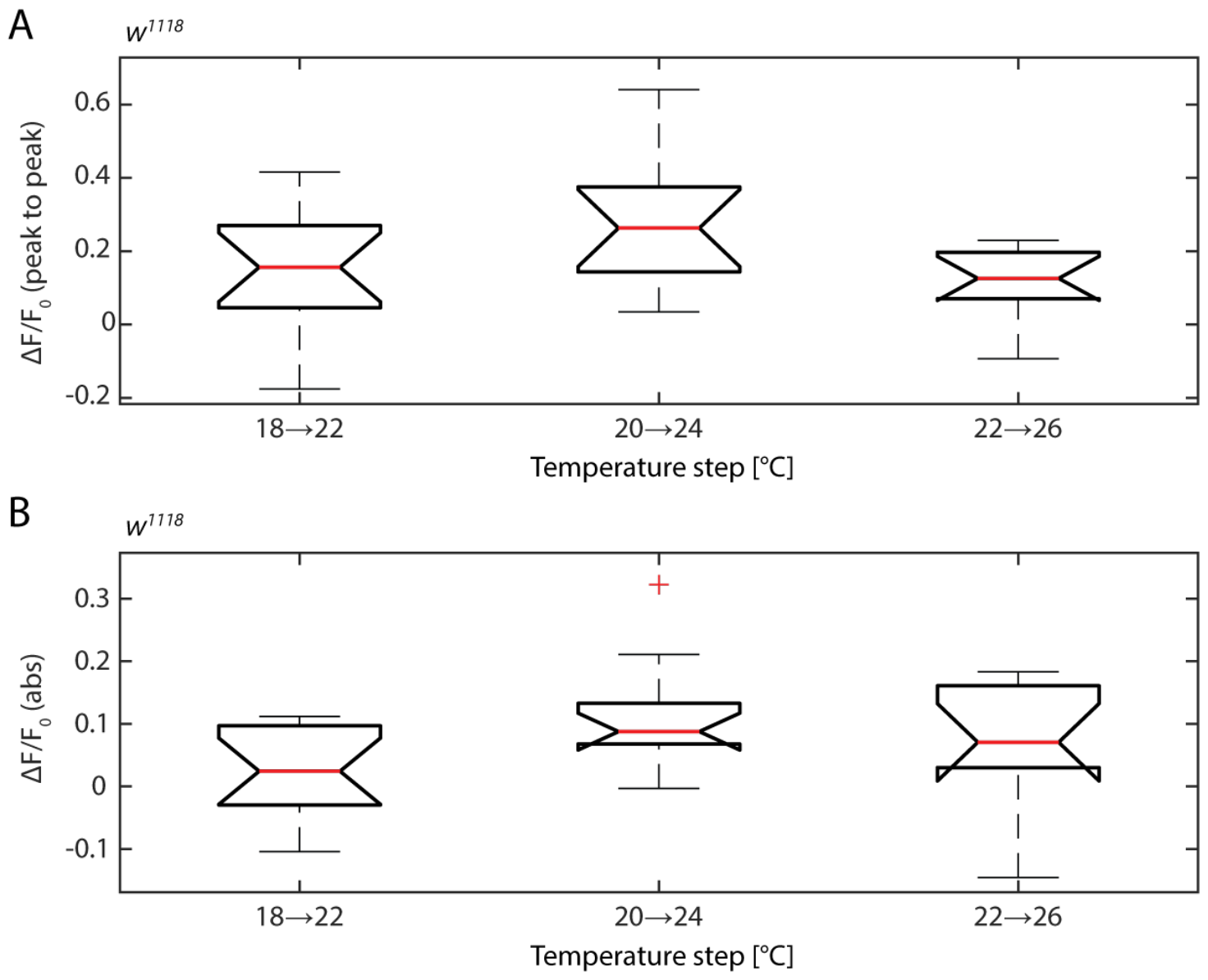

Figure 17: Comparison of $\mathrm{HC} \mathrm{Ca}^{2+}$ responses to temperature steps of same relative amplitude Temperature steps in which the relative temperature change equals $+4^{\circ} \mathrm{C}$ occur at 3 different absolute temperature changes in the stimulus protocols described in Figure 10: At the steps from 18 to $22^{\circ} \mathrm{C}$, from 20 to $24^{\circ} \mathrm{C}$ and at the step from 22 to $26^{\circ} \mathrm{C}$. The boxplots show the $\mathrm{HC} \Delta \mathrm{F} / \mathrm{F}_{\mathrm{O}}$ responses of $\mathrm{w}^{1118}$ flies to the three different temperature steps of $+4^{\circ} \mathrm{C}$. Red lines indicate median values, black boxes denote the upper and lower quartile ( $50 \%$ of the dataset) around the median. If no outliers are present, the whiskers denote $100 \%$ of the dataset, if outliers are present, whiskers denote the 1.5 interquartile distance. Outliers are marked a red crosses. Notches of the boxes denote the $95 \%$ confidence interval of the median. A) Change of $\Delta \mathrm{F} / \mathrm{F}_{0}$ value from the peak response of the previous step. B) The absolute value of $\Delta \mathrm{F} / \mathrm{F}_{0}$ peak during the respective temperature steps.

ative temperature changes of $-6^{\circ} \mathrm{C}$. The $\mathrm{CC}$ population shows its peak inhibitory response at $+6^{\circ} \mathrm{C}$ and its peak excitatory response and $-8^{\circ} \mathrm{C}$.

By fitting a sigmoid function to the data, the correlation between response amplitude and relative temperature step can be further illustrated. A sigmoid fit appears to match the data distribution of both control strains, although the shape of the fit appears somewhat more linear for the HC population of CantonS flies (Figure 20).

Additionally, if the arista neurons serve the role of relative temperature sensors, prolonged exposure to a temperature change would eventually result in an adaptation pro- 

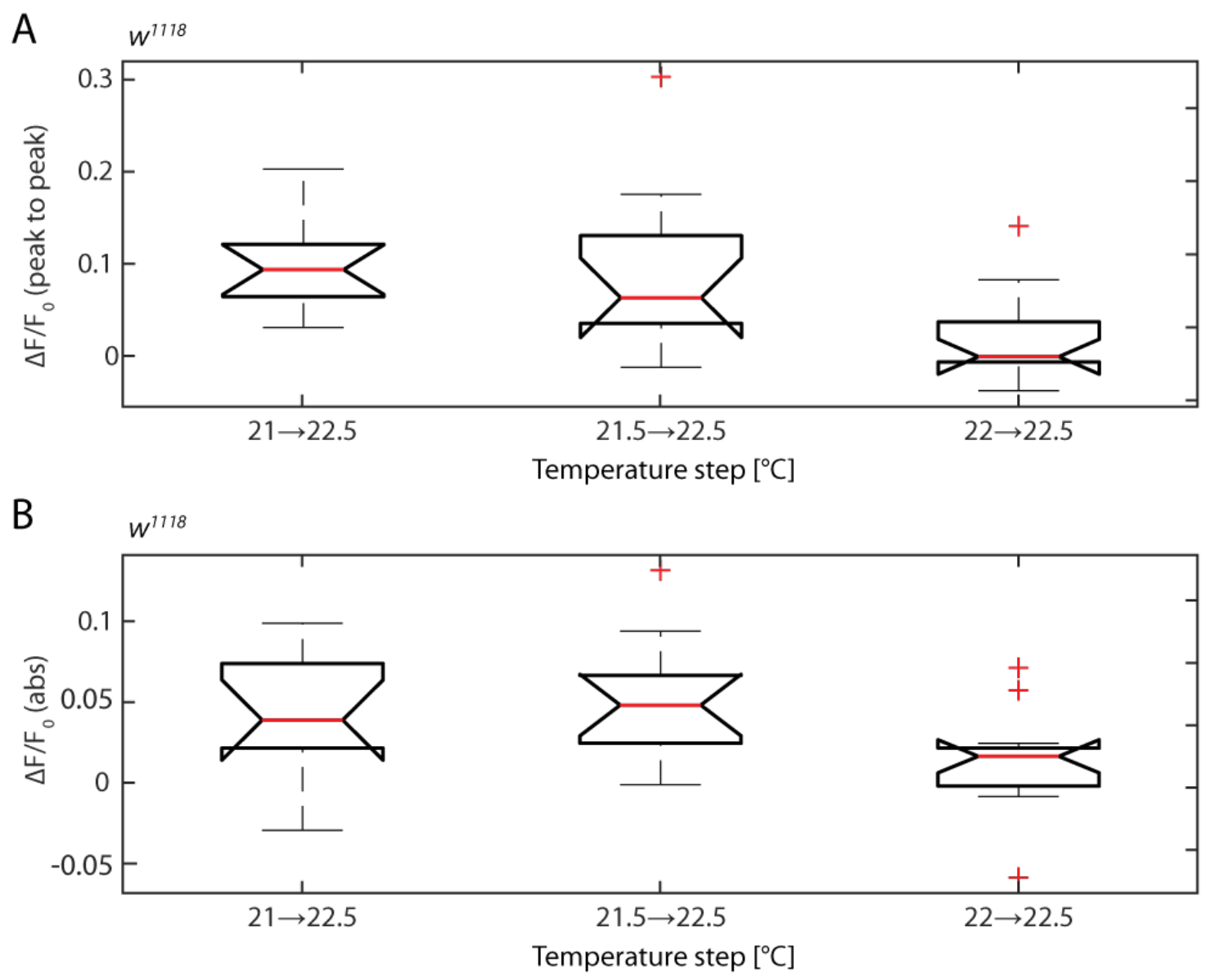

Figure 18: Comparison of $\mathrm{HC} \mathrm{Ca}^{2+}$ responses to temperature steps of different relative amplitudes . Temperature changes to an absolute temperature of $22.5^{\circ} \mathrm{C}$ occur with 3 different relative temperature changes in the stimuli shown in $10 \mathrm{~A}-\mathrm{D}$ : at $21-22.5^{\circ} \mathrm{C}$ (a change of $\left.1.5^{\circ} \mathrm{C}\right)$, at 21.5 to $22.5^{\circ} \mathrm{C}\left(1^{\circ} \mathrm{C}\right.$ relative change) and at 22 to $22.5^{\circ} \mathrm{C}\left(0.5^{\circ} \mathrm{C}\right.$ relative change). Different response amplitudes between the different temperature steps illustrate that the deciding factor for $\mathrm{Ca}^{2+}$ response amplitude does not appear to be the absolute temperature value. A) Change of $\Delta F / F_{0}$ value from the peak response of the previous step. A clear trend towards higher responses with stronger, realtive temperature changes is visible. Non-overlapping confidence intervalls between the 21 to $22.5^{\circ} \mathrm{C}$ and the 22 to $22.5^{\circ} \mathrm{C}$ steps indicate a significant difference. B) The absolute value of $\Delta F / F_{0}$ peak during the respective temperature steps.. Different response amplitudes between the different temperature steps illustrate that the deciding factor for $\mathrm{Ca}^{2+}$ response amplitude does not appear to be the absolute temperature value.

cess (for a review on the topic of adaptation in sensory systems, refer for example to Wark, Lundstrom, and Fairhall 2007). However, whether such an adaptation can be observed in the temperature evoked $\mathrm{Ca}^{2+}$ responses was not known. I therfore aimed to investigate if any potential adaptation returns the activity of the neurons to the baseline level or if part of the response has a tonic component.

To gain a better understanding of adaptation processes and time-courses in the HCs and CCs, I assessed the $\mathrm{HC}$ and CC responses of $\mathrm{w}^{1118}$ control flies, using the temperature 


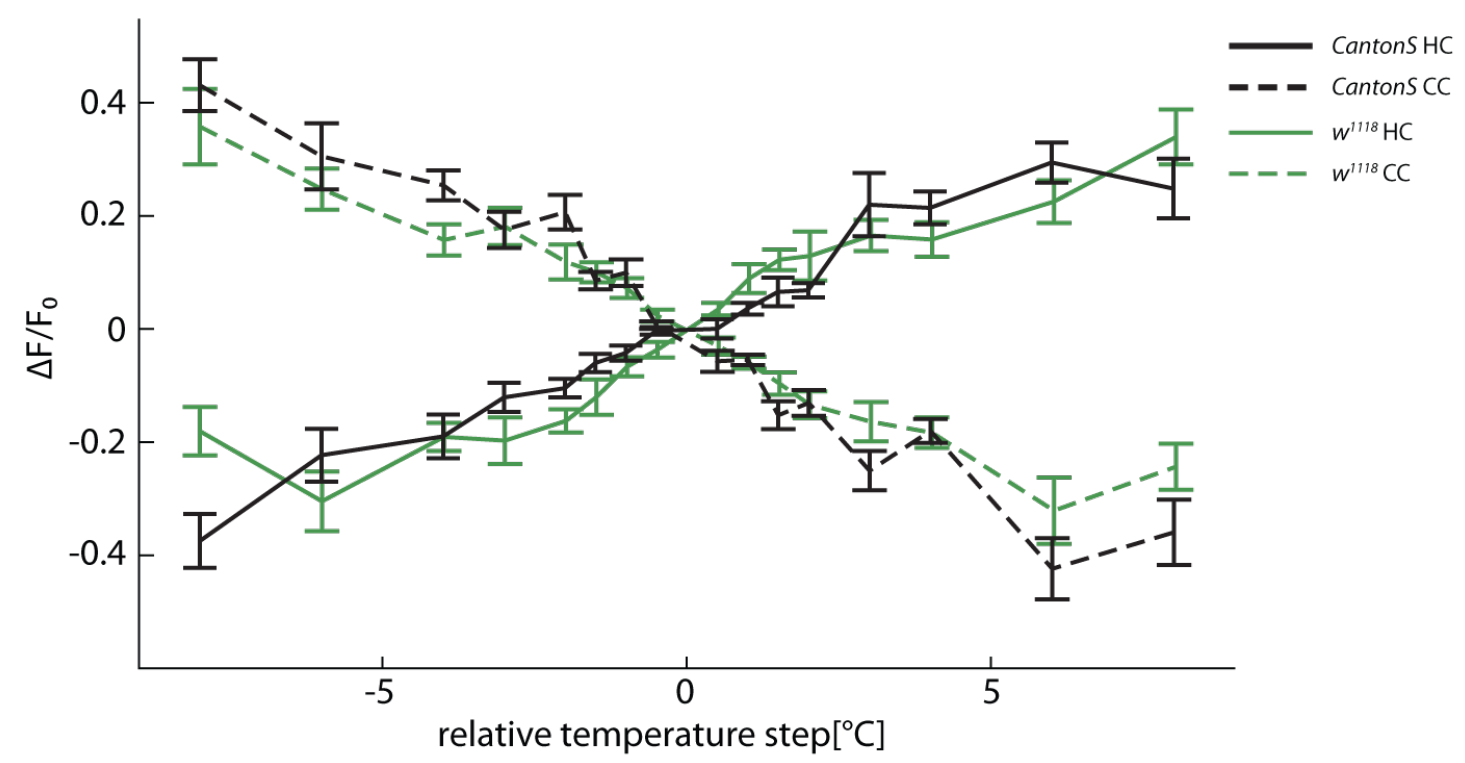

Figure 19: $\mathrm{Mean} \mathrm{Ca}^{2+}$ responses to relative temperature steps Mean $\Delta \mathrm{F} / \mathrm{F}_{0}$ responses of the arista neurons of the Cantons and $w^{1118}$ control strains to relative temperature changes applied during the different temperature step protocols. Cantons shown in black, W' 1118 in green. HC responses shown with solid lines, CC responses with dashed lines. Error bars denote SEM. Both HC and CC populations show a stronger change in fluorescence, the stronger the relative temperature change, with $\mathrm{HCs}$ showing an increase in $\Delta \mathrm{F} / \mathrm{F}_{\mathrm{O}}$ in response to temperature increments and a decrease in fluorescence to temperature decrements. CCs exhibit the oposite response behaviour. Both control strains show a similar response behaviour in both $\mathrm{HC}$ and $\mathrm{CC}$ populations.

stimuli described in Figure $10 \mathrm{E}-\mathrm{F}$, in which a single temperature step of either $26^{\circ} \mathrm{C}$ or $18^{\circ} \mathrm{C}$ is applied for 5 minutes, before the temperature returns to the starting value of $22^{\circ} \mathrm{C}$. $w^{1118}$ were used, as they exhibited no substantial differences from Cantons controls in previous experiments and the line generated considerably more offspring.

Both HC and CC population exhibited apparently constant levels of activity/inhibition (depending on stimulus direction) for the entire 5 minutes(Figure 21), with the exception of a transient response in the preferred stimulus direction. When exposed to a temperature drop to $18^{\circ} \mathrm{C}$ (a relative change of $-4^{\circ} \mathrm{C}$ ), the $\mathrm{CC}$ population exhibited an initially rapid increase in $\Delta \mathrm{F} / \mathrm{F}_{0}$ signal (Figure $21 \mathrm{~A}$ ), as was to be expected from previous experiments. I fitted an exponential function $\left(f(x)=a \cdot e^{-b \cdot x}+c\right)$ to the relevant response part which rendered a $\tau\left(\tau=\frac{1}{b}\right.$ ) of 163 seconds for $\mathrm{HC}$ and 407 seconds for CC in preferred temperature direction. After reaching a peak amplitude about 30 s before the timepoint in which the temperature curve reaches $18^{\circ} \mathrm{C}$, fluorescence intensity began to drop again. However, after a time frame of approximately 50 s, fluorescence decay began to stall. The $\mathrm{Ca}^{2+}$ signal even appears to reach a plateau like constant level, that lay above a $\Delta F / F_{0}$ value of 0.15 , well above the starting value. Once temperature began to rise back to the baseline temperatue of $22^{\circ} \mathrm{C}$ again, the $\Delta \mathrm{F} / \mathrm{F}_{0}$ signal showed a fast drop to around -0.08 , which 


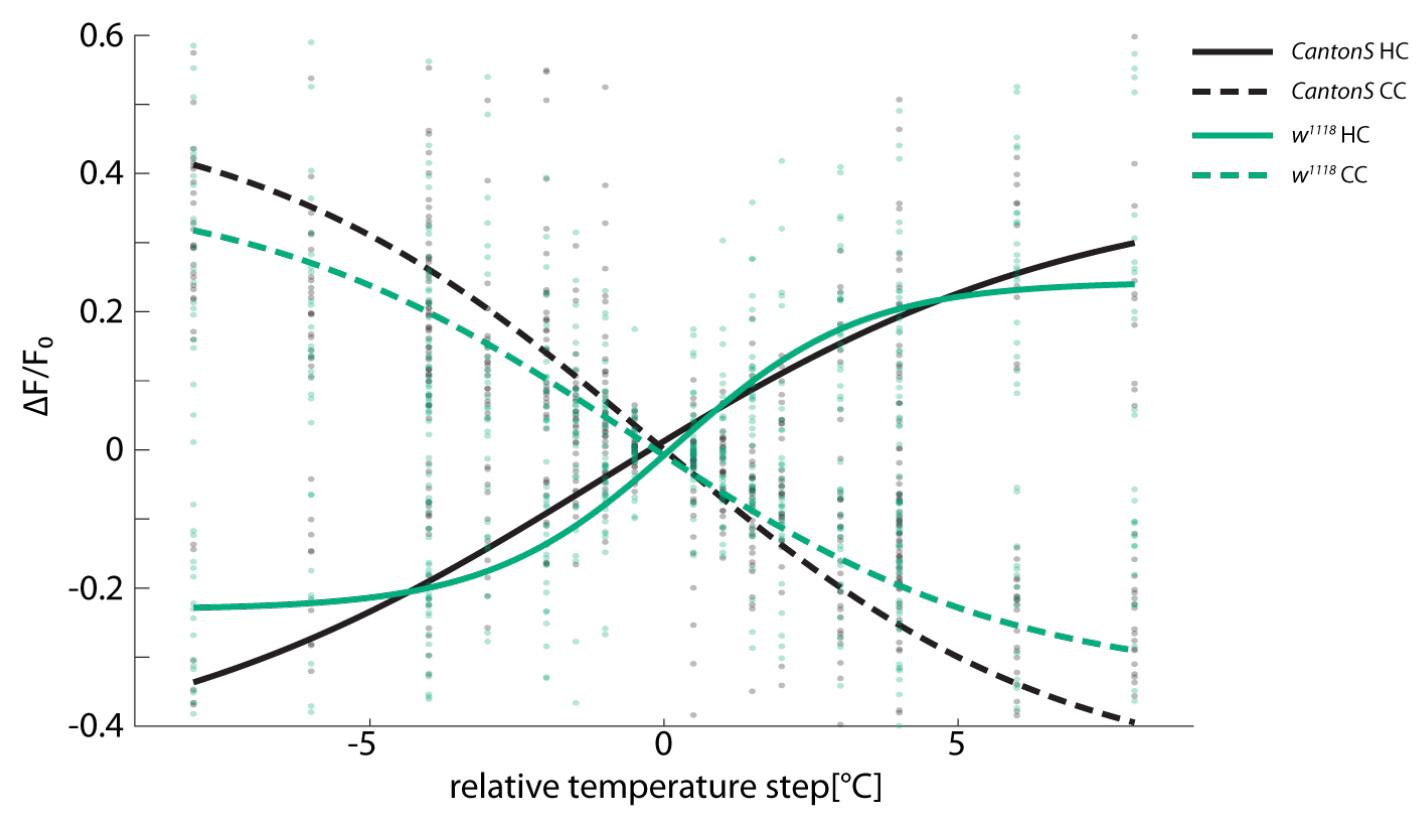

Figure 20: Sigmoid fits to relative temperature step responses of wildtype control flies Fits to the $\Delta F / F_{0}$ response values of both CantonS and $w^{1118}$ control strains. Cantons shown in black, $w^{1118}$ in green. Dots mark single measurements of all tested HCs and CCs for the respective strains. Fits to $\mathrm{HC}$ responses shown as solid lines, fits to $\mathrm{CC}$ responses shown as dashed lines. Both $\mathrm{HC}$ and $\mathrm{CC}$ responses show a clear increase in response amplitude with increasing temperature step amplitude. As shown before, clear excitatory responses of $\mathrm{HCs}$ to temperature increments. and inhibitory responses to temperature decrements. CCs react vice versa. Both fits appear to follow a sigmoid shape in the temperature ranges that were tested in this study.

adapted back to around baseline level over the course of $75 \mathrm{~s}$. This indicates a considerable tonic component of the calcium signal, while the "overshoot" of the response after returning to the $22^{\circ} \mathrm{C}$ base temperature illustrates the relative response coding, as the return to the previous temperature seems to be reacted to as a "cold step". As inferred from the previous experiments, the $\mathrm{HC}$ population showed a reduction in the $\Delta \mathrm{F} / \mathrm{F}_{0}$ signal, in response to the cold stimulus (Figure $21 \mathrm{~A}$ ). While the $\mathrm{HC}$ responses showed a signal change that was comparable in speed to the CCs at the onset of the cold stimulus, I could observe no obvious adaptation over time and the signal appeared to stay at a constant plateau level at about -0.3 to -0.35 .

At the end of the temperature stimulus, a similar response behaviour to the CCs could be observed, in that the $\Delta F / F_{0}$ signal rose quickly and "overshot" the 0 level to a value just under 0.2, after which the signal adapted over a similar timecourse like the CCs to about baseline. When studying the responses of $\mathrm{HC}$ and $\mathrm{CC}$ to a prolonged temperature increment stimulus, I could observe a similar response behaviour as with the temperature decrement (Figure $21 \mathrm{~B}$ ): HCs react in an excitatory manner to the temperature increase and, after an initial peak, adapt to a constant level (of just under 0.1). The CC response showed no initial peak in a temperature increase, and instead exhibited a constant de- 

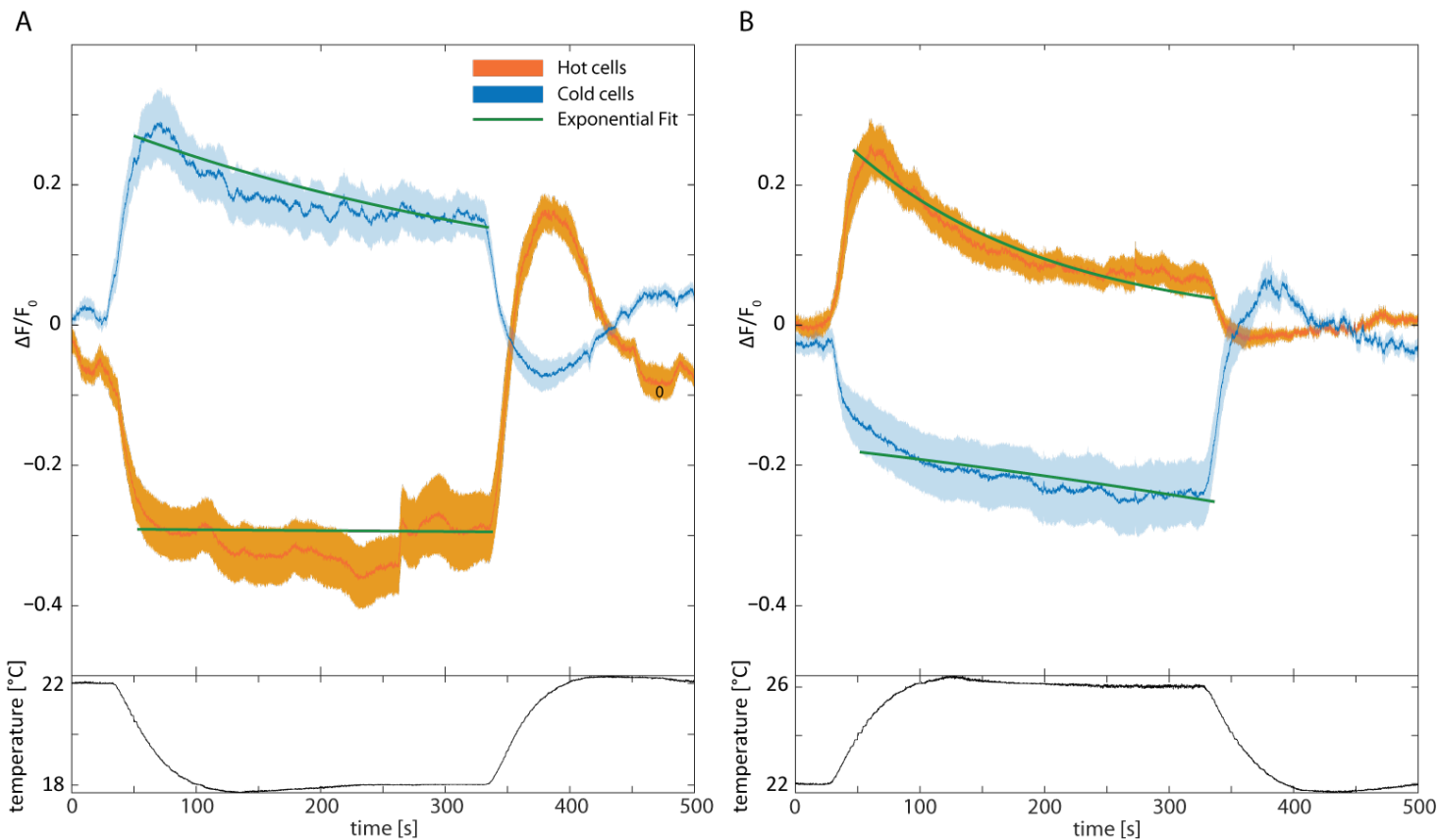

Figure 21: Calcium response of arista neurons to prolonged temperature change $\Delta F / F_{0}$ responses of the arista neurons of $W^{1118}$ control flies to 300 s second temperature stimuli. After the temperature steps, the temperature was returned to starting temperature of $22^{\circ} \mathrm{C}$. $\mathrm{HC}$ responses are shown in orange, $\mathrm{CC}$ responses in blue. Mean response is represented with a solid lane, shaded areas denote SEM. An exponential curve to the response during temperature stimulation is fitted to the $\Delta \mathrm{F} / \mathrm{F}_{0}$ signal of both cell types. A) Response of $\mathrm{HC}$ and CC populations to a temperature step of $18^{\circ} \mathrm{C}$ for $300 \mathrm{~s}$. $\tau$ of $\mathrm{HC}$ fit: 17414s, $\tau$ of CC fit:407 s. n: $\mathrm{HC}=13$; $\mathrm{CC}=15 \mathrm{~B})$ Response of $\mathrm{HC}$ and CC populations to a temperature step of $26^{\circ} \mathrm{C}$ for 300s. $\tau$ of HC fit: $163 \mathrm{~s}, \tau$ of CC fit: $884 \mathrm{~s}$. $\mathrm{n}: \mathrm{HC}=12$; CC=15

crease, which however slowed down considerably over time. At the end of the stimulus step, in which the temperature returns to the starting value of $22^{\circ} \mathrm{C}$, the $\mathrm{CCs}$ again show the "overshoot" that was also observed for both cell types in the previous stimulus condition, before adapting again to a value close to the starting value (Figure $21 \mathrm{~B}$ ).

These findings suggest a substantial tonic part of the response of HCs and CCs to temperature steps. Intriguingly, in the experimental conditions tested here, I could only observe adaptation processes of the $\Delta F / F_{0}$ responses in the "preferred stimulus direction" of the arista neurons, namely temperature increases for $\mathrm{HCs}$ and decrease in temperature for CCs. This is espically confounding as the previous experiments strongly hinted at relative coding properties, making fast adaptation necessary. Adaptation analysis now suggests a strong tonic component. 


\subsection{Effect of NOMPC on calcium responses in arista neu- rons}

Based on my physiological characterization of the thermosensitive arista neurons, I could now compare their responses to those of nompC mutant flies unravel the function of a mechano-transducer in a thermosensitive cell.

I assesed the effect of different nompC mutations and nompC overexpression on the temperature evoked $\mathrm{Ca}^{2+}$ responses. I used flies carrying the nompC $\mathrm{C}^{3}$ null mutation (in which a point mutation leads to a premature stop codon), both heterozygously and homozygously, as well as the weaker nomp $\mathrm{C}^{\text {f00914 }}$ mutation, which shows a reduction in NOMPC protein amount, (Lee, Moon, et al. 2010). To increase the amount of NOMPC, I utilized flies expressing an additional functional copy of the nompC gene under UAS control in the wildtype background. I used the same constructs in the homozygous nomp $C^{3}$ background to serve as a functional rescue. All fly lines were exposed to the 4 different temperature stimulus paradigms described in Figure $10 \mathrm{~A}-\mathrm{D}$. To control for possible effects of the nompC-Gal4 or UAS-nompC constructs, I also assessed the temperature evoked $\mathrm{Ca}^{2+}$ responses of flies carrying only the Gal4 or only the UAS constructs, both in the wildtype and nomp $\mathrm{C}^{3}$ background. As these lines only served as additional controls, they were exposed to one of the temperature step stimuli, with an increasing amplitude of temperature stimulation (Stimulus shown in Figure $10 \mathrm{D}$ ). Neither the nompC-Gal4 nor the UAS-nompC constructs affected the $\Delta \mathrm{F} / \mathrm{F}_{0}$ response amplitude in both the wildtype and the nompC $\mathrm{C}^{3}$ background (for the $\mathrm{Ca}^{2+}$ response curves, see Appendix Figures $\mathrm{A} 12$, page 105).

\subsubsection{Mutations of nompC lead to a reduction in $\mathrm{HC}$ response amplitude}

Flies carrying a homozygous nomp $\mathrm{C}^{3}$ mutation exhibited a distinctly lower response amplitude in the $\mathrm{HC}$ population, with the mean response amplitude of HCs staying below a $\Delta F / F_{0}$ value of 0.1 (Figure $22 \mathrm{~B}$ ). While the amplitude of the $\mathrm{CC}$ population also appeared to be affected, this change in response amplitude was not as strong as in the HCs, with mean excitatory responses reaching a $\Delta \mathrm{F} / \mathrm{F}_{0}$ of 0.2 (Figure $23 \mathrm{~B}$ ). The stimulus direction selectivity is preserved in both cell populations. The stark reduction in response amplitude also appears to affect both excitatory and inhibitory parts of the $\mathrm{HC}$ responses, as the drop in $\Delta F / F_{0}$ signal elicited by temperature decrements also appears to be not as pronounced as in the wildytpe controls (Figure $22 \mathrm{~B}$ ). When fitting a sigmoid function to the dataset, it is much more shallow and linear than in wildtype controls (Figure 24 B and Figure $25 \mathrm{~B}$ ). Comparing the gain of the $\Delta \mathrm{F} / \mathrm{F}_{0}$ signal per ${ }^{\circ} \mathrm{C}$ of temperature change, in the case of the strongest temperature steps per stimulus, revealed a significant difference between Cantons controls and the nompC ${ }^{3}$ mutants (Figure 26). The effect was visible in 
both the HC (Figure $26 \mathrm{~A}$ ) and CC population (Figure $26 \mathrm{~B}$ ), although the effect was not as strong in the CC signal, similarly to how it appeared in the mean response curves and sigmoid fits.

A

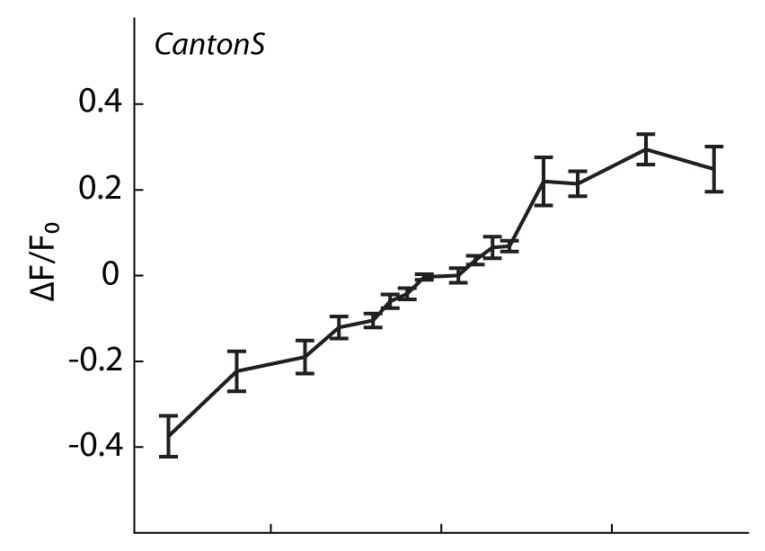

C

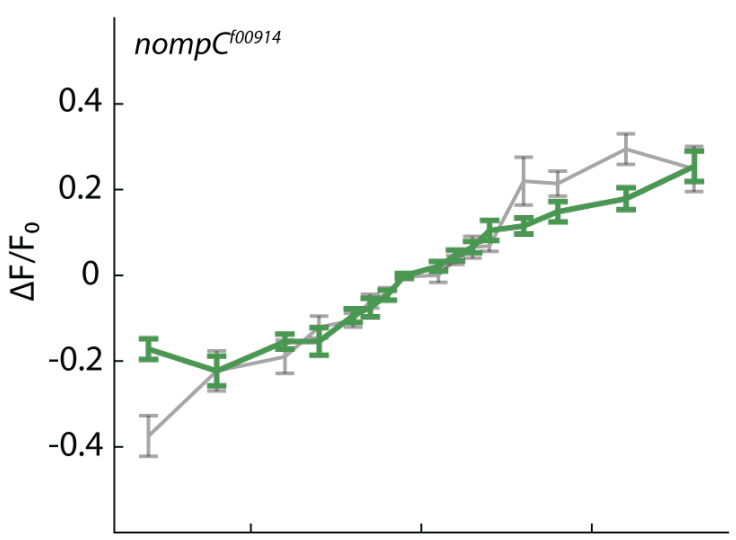

$\mathrm{E}$

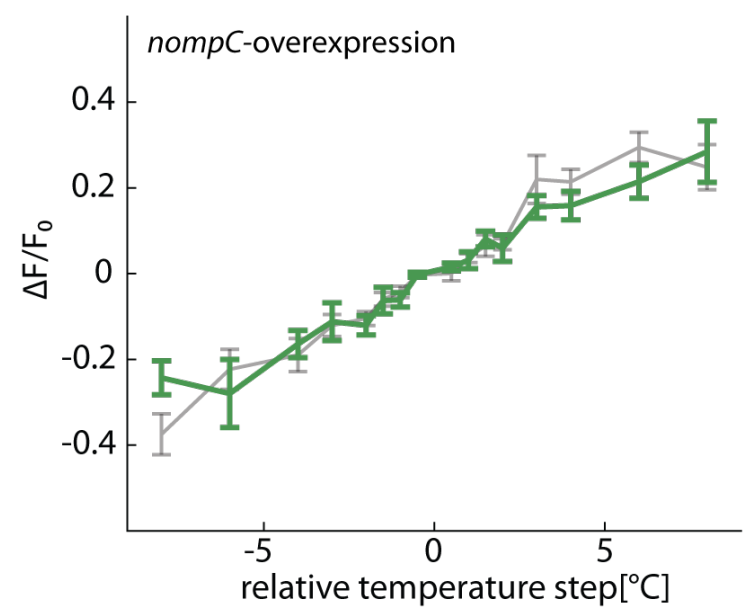

B

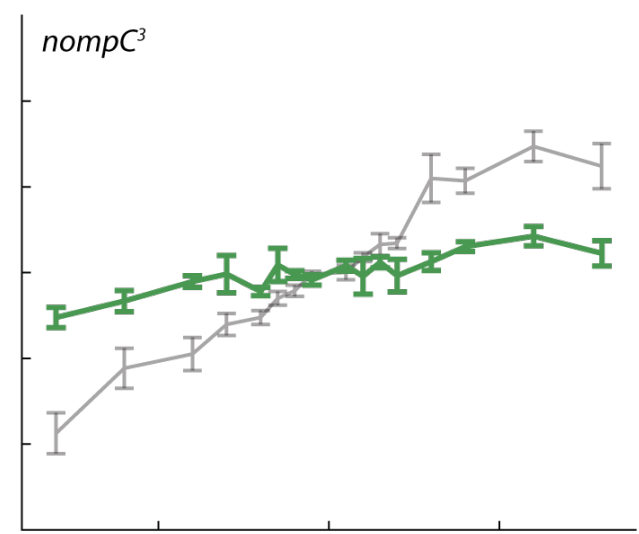

D

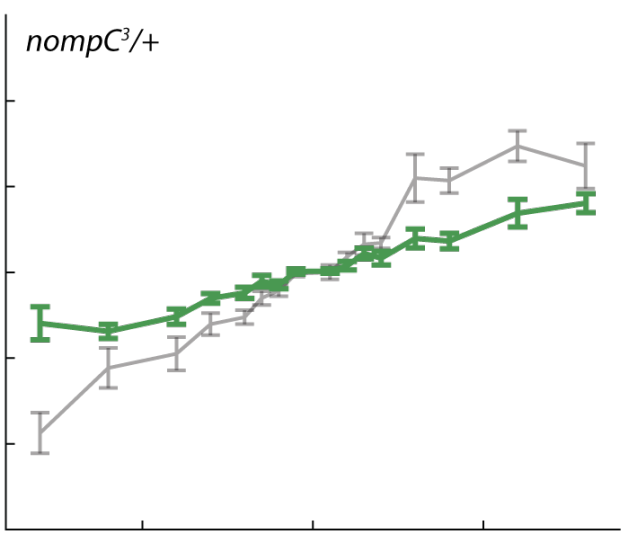

$\mathrm{F}$

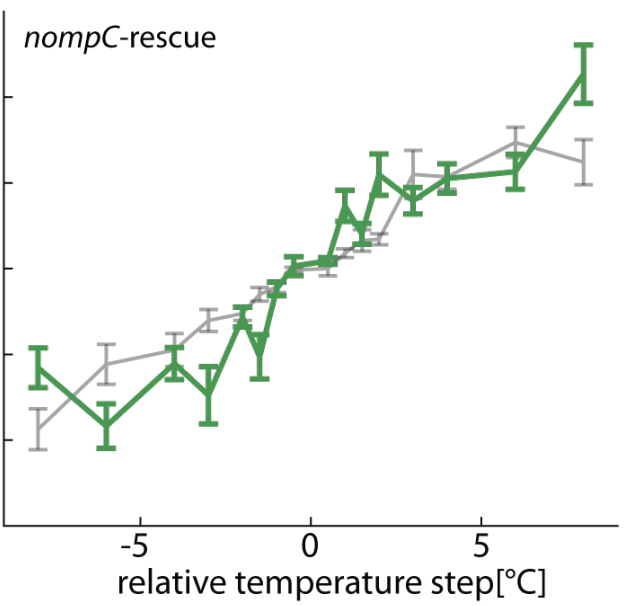


Figure 22: Mean $\mathrm{Ca}^{2+}$ responses of hot cells in nompC ${ }^{3}$ mutant, rescue and overexpression flies The mean values of the $\mathrm{HC}$ peak responses to each relative temperature step are shown. HCs exhibited excitatory responses to temperature increments and inhibitory responses to temperature decrements in all tested strains. The curve of the CantonS control strain is shown in each sub-plot in grey. Homozygous nompC ${ }^{3}$ (carrying a null mutation for the nompC gene) mutants show a flatter curve with overall lower response amplitudes to higher temperature changes. Heterozygous nomp $\mathrm{C}^{3}$ mutants, which still carry one functional copy of nompC, exhibit an intermediate phenotype compared to homzoygous mutants and wildtype controls. Mutants carrying the nompC ${ }^{\text {f00914 }}$ allele show a somewhat more linear mean response curve, with lower response amplitudes than wildtypes in the range of +3 to $+6^{\circ} \mathrm{C}$ relative temperature change. A) Mean response amplitude of CantonS flies to relative temperature changes. B) Mean responses of homozygous nomp $\mathrm{C}^{3}$ mutants, carrying a null mutation for nompC, which should therefore lack any functional protein of the channel. C) Mean responses of nompC f00914 mutant flies, which leads to a reduced amount of NOMPC protein. D) Mean responses of heterozygous nomp $\mathrm{C}^{3}$ mutants, which still retain one functional copy of the nompC gene. E) Mean responses of nompC-overexpression flies, carrying an additional nompC copy under UAS control. F) Mean responses of nompC-rescue flies, in which a nompC-gene under UAS control is used to rescue the nomp $C^{3}$ mutation. 
A

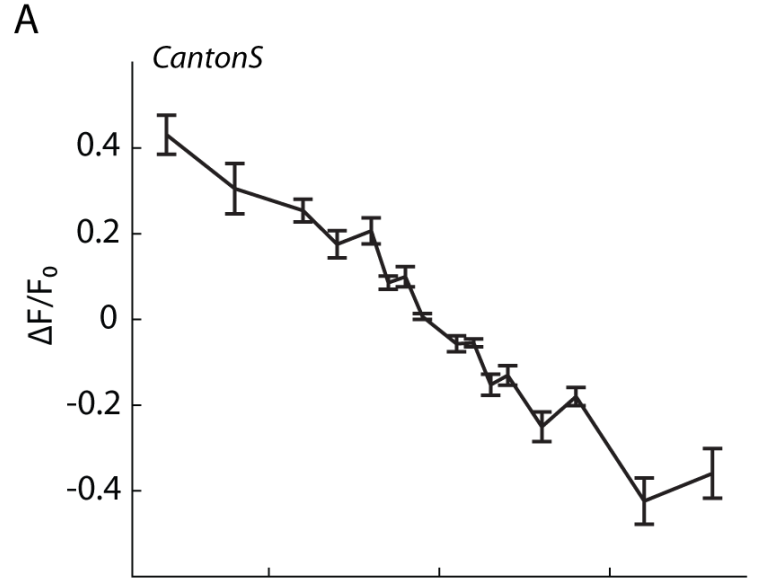

C

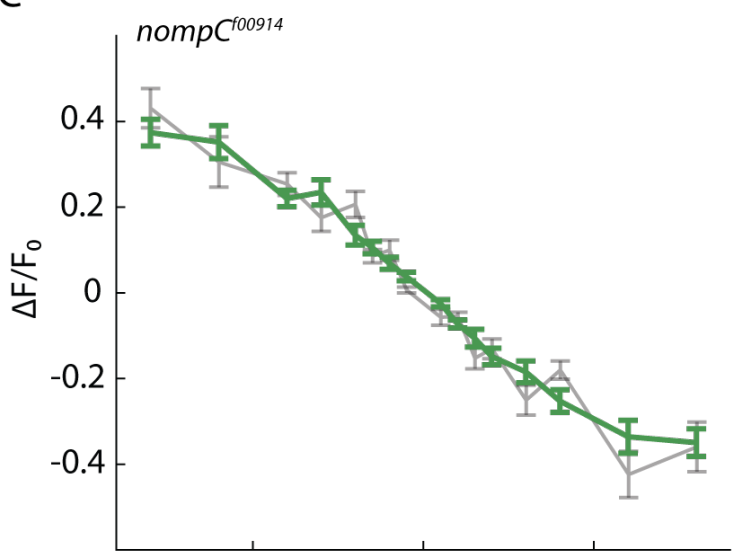

E

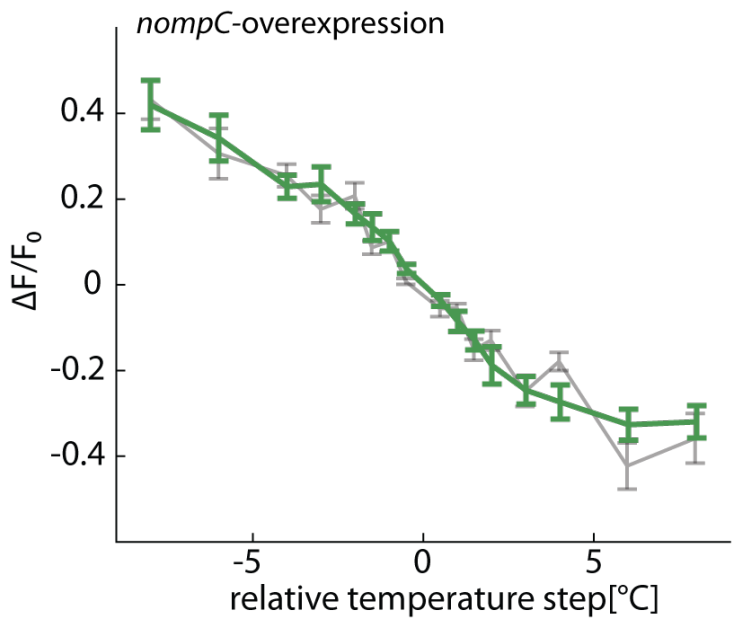

B

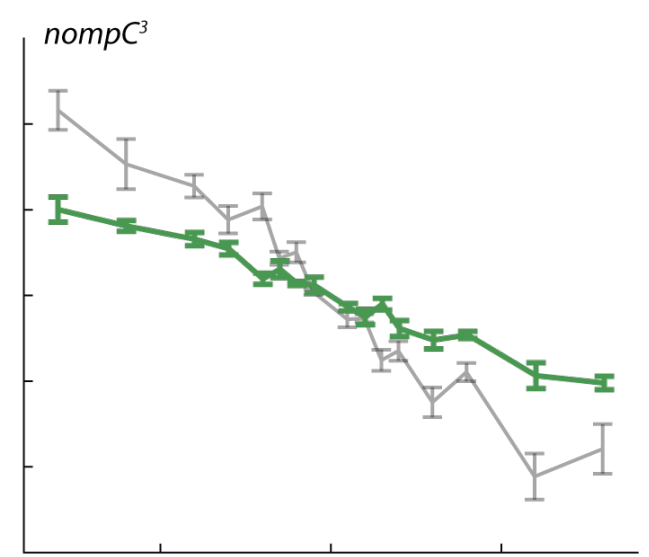

D

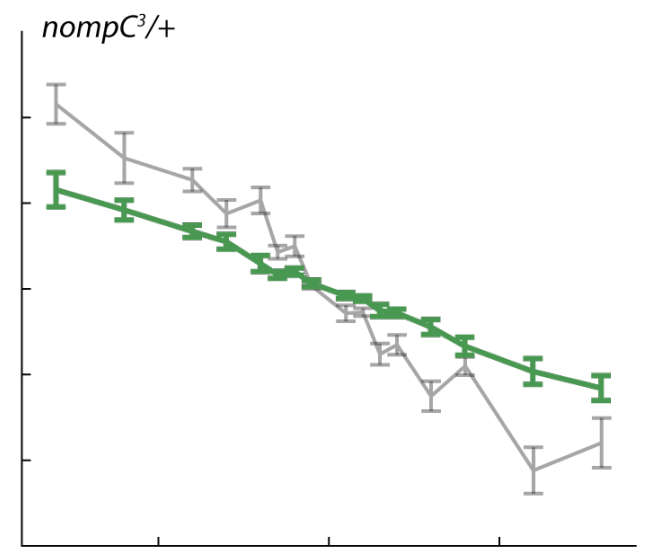

F

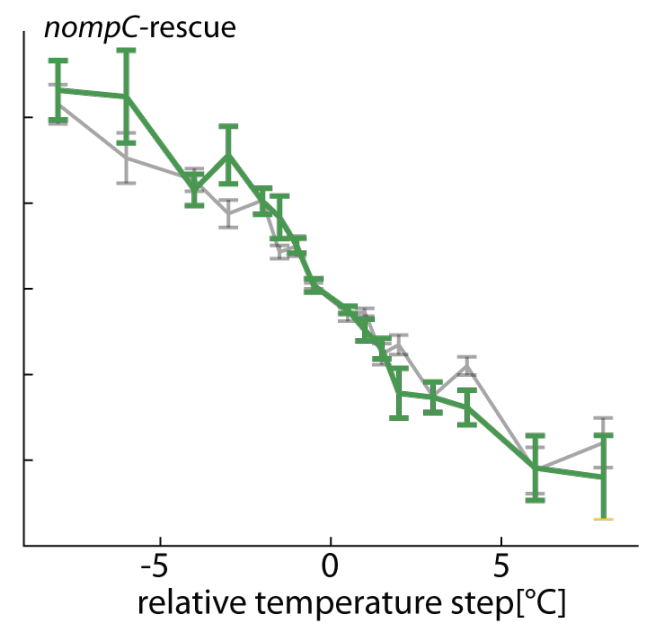


Figure 23: Mean $\mathrm{Ca}^{2+}$ responses of cold cells in nompC ${ }^{3}$ mutant, rescue and overexpression flies The mean values of the $\mathrm{CC}$ peak responses to each relative temperature step are shown. CCs exhibited excitatory responses to temperature decrements and inhibitory responses to temperature increments in all tested strains. The curve of the Cantons control strain is shown in each sub-plot in grey. nomp $\mathrm{C}^{3}$ mutants, carrying a null mutation of the nompC gene, again showed a reduction in response amplitude, although the effect did appear to be not as severe as in the $\mathrm{HC}$ population shown before. A) Mean response amplitude of CantonS flies to relative temperature changes. B) Mean responses of homozygous nomp $\mathrm{C}^{3}$ mutants, carrying a null mutation for nompC, which should therefore lack any functional protein of the channel. C) Mean responses of nompC ${ }^{f 00914}$ mutant flies, which leads to a reduced amount of NOMPC protein. D) Mean responses of heterozygous nomp $\mathrm{C}^{3}$ mutants, which still retain one functional copy of the nompC gene. E) Mean responses of nompC-overexpression flies, carrying an additional nompC copy under UAS control. F) Mean responses of nompC-rescue flies, in which a nompC-gene under UAS control is used to rescue the nomp $C^{3}$ mutation. 
A

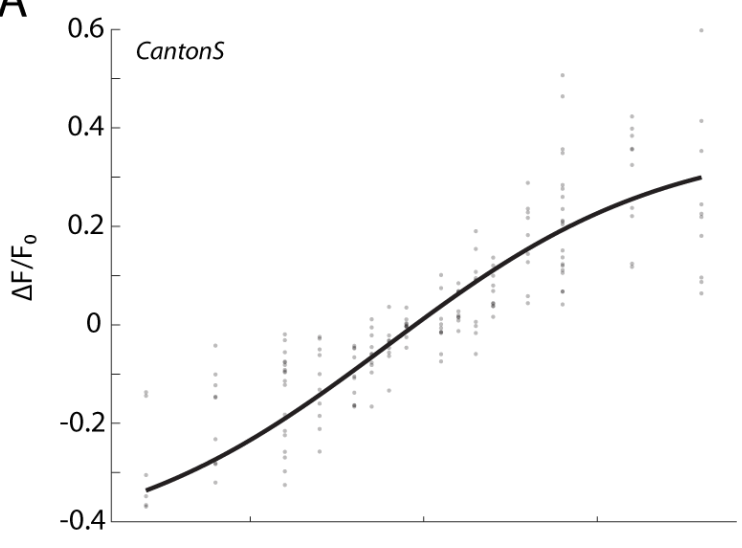

C

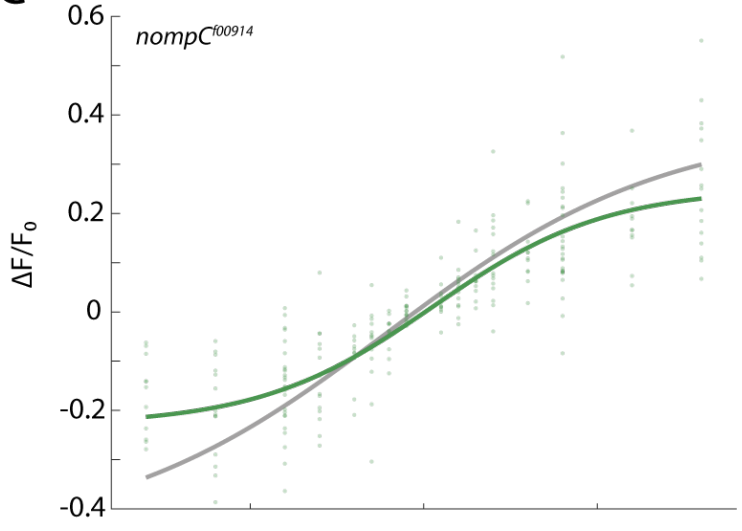

$\mathrm{E}$

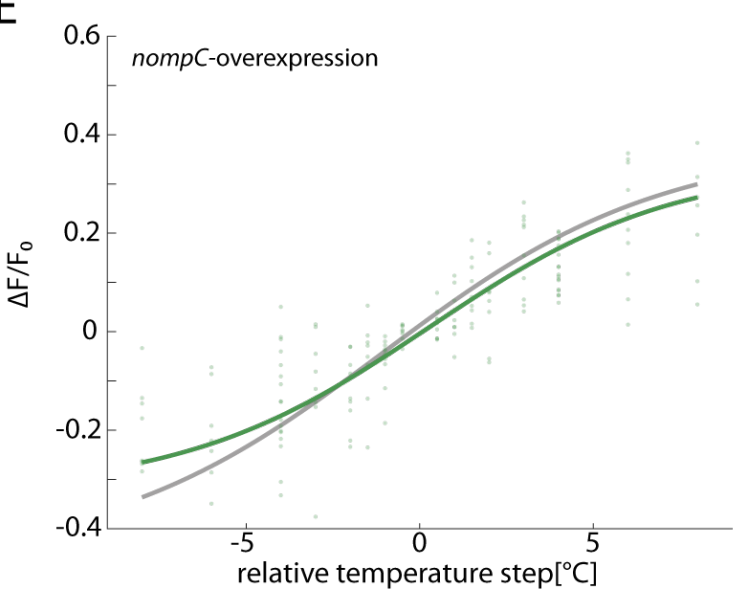

B

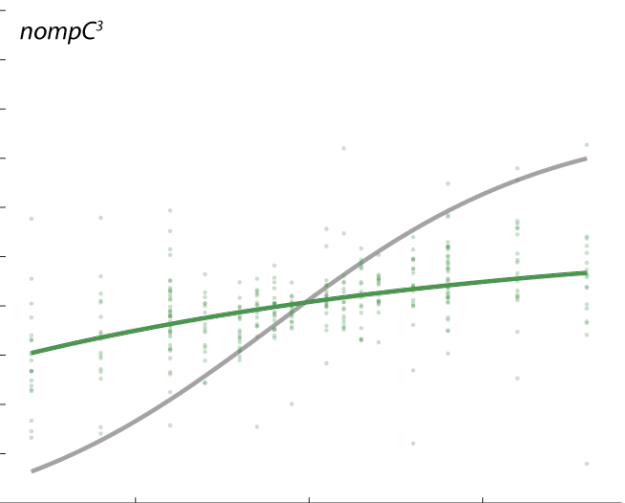

D

nomp $C^{3} /+$

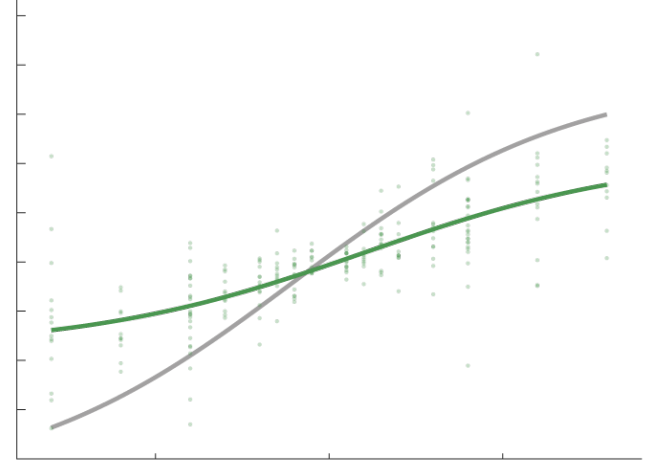

$\mathrm{F}$

nompC-rescue

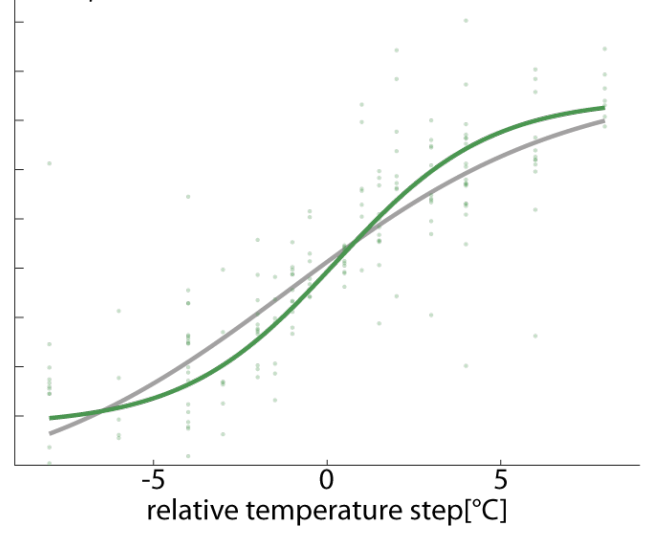

Figure 24: Sigmoid fits to relative temperature step hot cell responses Fits to the $\Delta \mathrm{F} / \mathrm{F}_{0}$ response values of hot cells to relative temperature changes. Dots indicate the $\Delta \mathrm{F} / \mathrm{F}_{0}$ response peaks of all tested cells of the respective flies. The fit to nomp $\mathrm{C}^{3}$ data again shows a more shallow gain than the wildtype controls. although the effect appears to be not as severe as in HCs. A) Fit to responses of Cantons flies to relative temperature changes. B) Homozygous nomp $\mathrm{C}^{3}$ mutants. C) nomp $\mathrm{C}^{\text {f00914 }}$ mutant flies. D) Heterozygous nomp $\mathrm{C}^{3}$ mutants. E) nompC-overexpression flies, carrying an additional nompC copy under UAS control. F) nompC-rescue flies, in which a nompC-gene under UAS control is used to rescue the nompC $C^{3}$ mutation. 

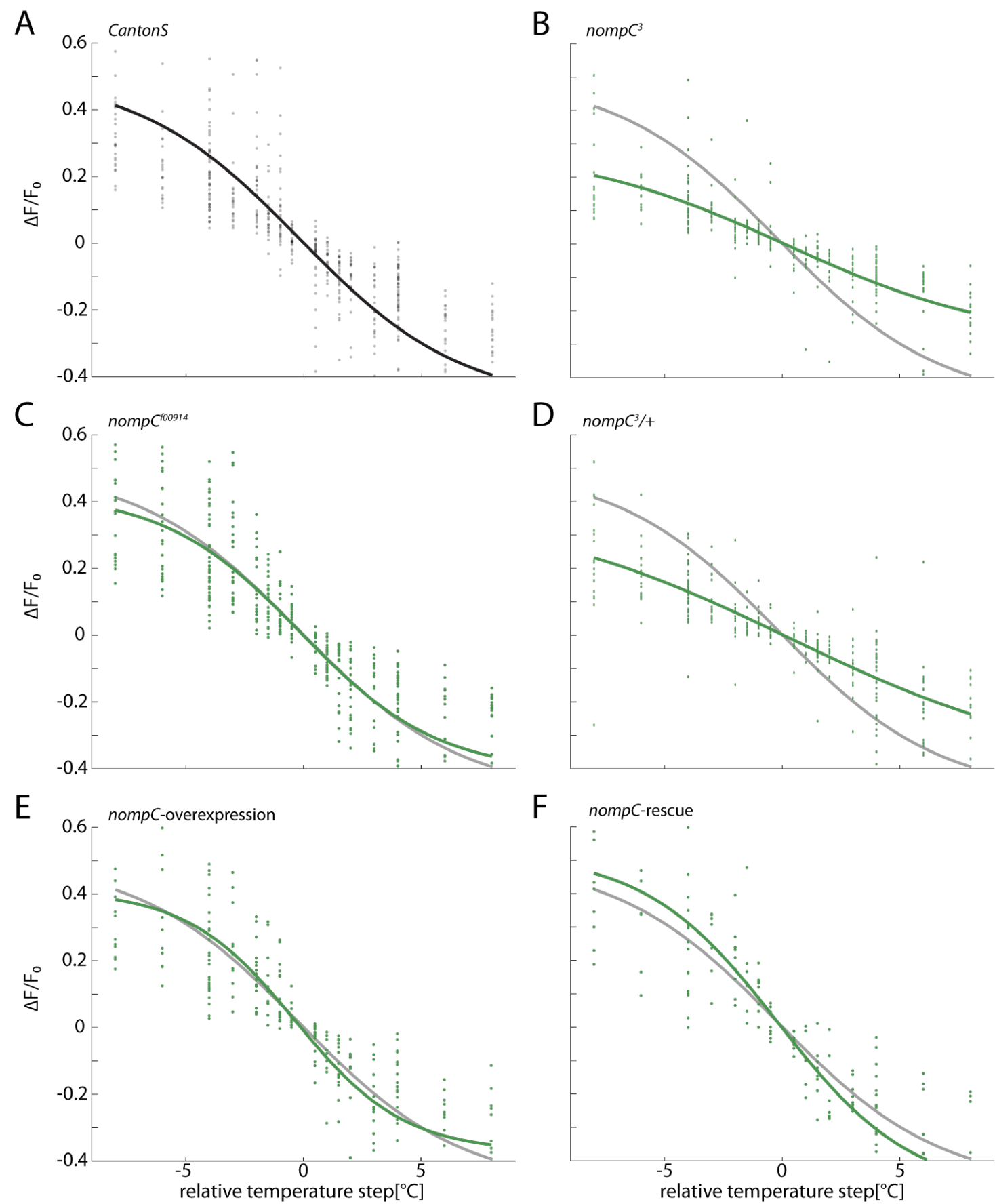

Figure 25: Sigmoid fits to relative temperature step cold cell responses Fits to the $\Delta F / F_{0}$ response values of cold cells to relative temperature changes. Dots indicate the $\Delta F / F_{0}$ response peaks of all tested cells of the respective flies. The fit to nomp $\mathrm{C}^{3}$ data clearly shows a much more shallow gain, illustrating a reduced response amplitude to higher temperature step amplitudes. Additionally, the shape of the fit is almost entirely linear, losing the sigmoid characterstic seen in wildtype flies. Heterozygous nomp $C^{3}$ mutants again exhibit an intermediate phenotype. A) Fit to responses of CantonS flies to relative temperature changes. B) Homozygous nompC $C^{3}$ mutants. C) nompC $\mathrm{f}^{\text {f0o914 }}$ mutant flies. D) Heterozygous nomp $\mathrm{C}^{3}$ mutants. E) nompC-overexpression flies, carrying an additional nompC copy under UAS control. F) nompC-rescue flies, in which a nompC-gene under UAS control is used to rescue the nomp $C^{3}$ mutation. 
A hot cells

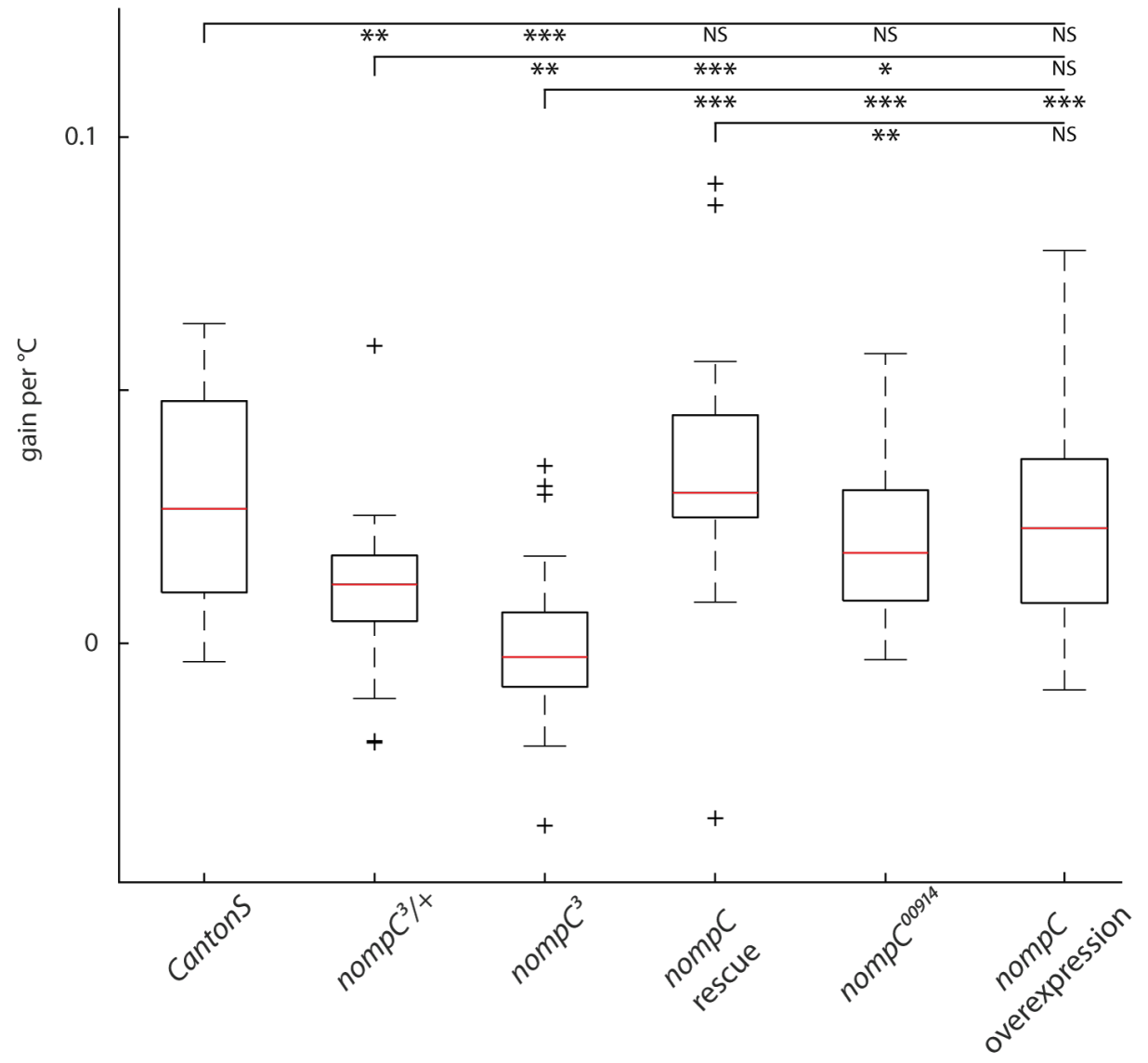

B cold cells

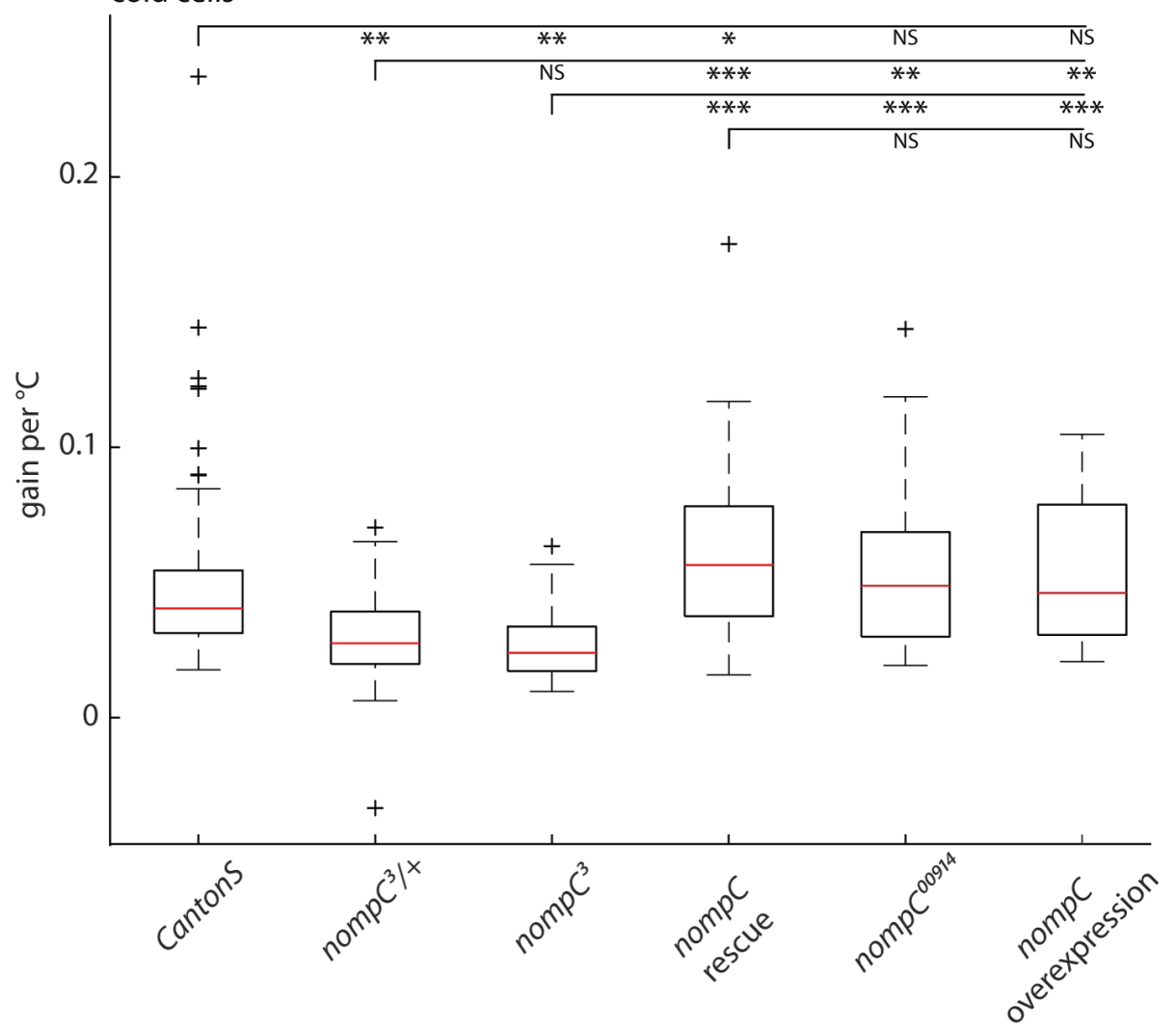


Figure 26: Comparison of $\Delta F / F_{0}$ gain per ${ }^{\circ} \mathrm{C}$ Shown is the gain of the $\Delta F / F_{0}$ per ${ }^{\circ} \mathrm{C}$ change, for the biggest step of the preferred stimulus direction (meaning a temperature increase for $\mathrm{HCs}$ and a decrease for CCs). Red lines denote median values. Boxplots presented as described in 2.7 (Page 31). Significances as: ${ }^{*}=p<0.05 ;{ }^{* *}=p<0.01 ;{ }^{* * *}=$ $\mathrm{p}<0.001$; NS: not significant. A) $\Delta \mathrm{F} / \mathrm{F}_{0}$ gain per ${ }^{\circ} \mathrm{C}$ of $\mathrm{HCs}$ for the largest, relative temperature increase step (depending on stimulus protocol this means either +6 or $+8^{\circ} \mathrm{C}$ ). nomp $\mathrm{C}^{3}$ mutants (both homo- and heterozygously) differ significantly from wildtype controls. Rescue of the mutation via Gal4-UAS restores value to wildtype levels. Neither the nomp $\mathrm{C}^{\mathrm{f00914}}$ mutation, nor overexpression of nompC altered the $\mathrm{HC}$ gain per ${ }^{\circ} \mathrm{C}$ significantly from Cantons controls. B) $\Delta \mathrm{F} / \mathrm{F}_{0}$ gain per ${ }^{\circ} \mathrm{C}$ of $\mathrm{CCs}$ for the largest, relative temperature decrease step (depending on stimulus protocol this means either -6 or $-8^{\circ} \mathrm{C}$ ). Although the effect is not as strong as in the $\mathrm{HC}$ population, $\mathrm{CC}$ gain per ${ }^{\circ} \mathrm{C}$ of nompC $\mathrm{C}^{3}$ mutants still differs significantly from CantonS controls. Rescue of the mutation does not only restore $\mathrm{CC}$ gain to wildtype levels, but ascutally appears to increase the response, as the gain per ${ }^{\circ} \mathrm{C}$ is significantly higher than in wildtype controls. nompC $\mathrm{f00914}^{\text {mutation or }}$ overexpression of nompC did again not show a significant difference.

When assessing the response of Drosophila carrying a heterozygous nomp $\mathrm{C}^{3}$ mutation, an intermediate effect on the $\mathrm{HC}$ response amplitude could be observed: The HC response amplitude was lowered, when compared to control flies, however the effect was not as strong as in the homozygous mutant flies, as mean $\mathrm{HC}$ response reached a maximum $\Delta \mathrm{F} / \mathrm{F}_{\mathrm{O}}$ value of above 1.5 and the $\mathrm{CC}$ reached a maxiumum exciatory response of around 2.5 (Figure 22 D and Figure $23 \mathrm{D}$ ). Similarly, plotting a fit to the dataset reveals a gain and curve shape between the wildytype controls and the nompC $\mathrm{C}^{3}$ mutants (Figure $24 \mathrm{D}$ and Figure $25 \mathrm{D}$ ). The comparison of $\Delta \mathrm{F} / \mathrm{F}_{0}$ gain per ${ }^{\circ} \mathrm{C}$ pointed towards the same result, with a significant difference between nomp $\mathrm{C}^{3} /+$ flies and Cantons controls, although not as significant as in the case of homozygous nompC $\mathrm{C}^{3}$ mutants (Figure 26 A). Furthermore, the $\mathrm{HC}$ gain per ${ }^{\circ} \mathrm{C}$ of nompC ${ }^{3} /+$ was also significantly different from the homozygous nomp $\mathrm{C}^{3}$ mutants. This could be a first implication that the number of functional nompC gene copies has a direct effect on the temperature evoked responses of the arista HCs.

Furthermore, I assessed the temperature responses of nompC f00914 mutant flies. Drosophila of this line exhibited an reduction in $\mathrm{HC}$ response amplitude, though not as pronounced as in homozygous or heterozygous nomp $\mathrm{C}^{3}$ mutants. Mean response amplitude of the $\mathrm{HCs}$ appears to show a slightly more linear curve, with lower response amplitudes in the range of +3 to $+6^{\circ} \mathrm{C}$ relative temperature steps (Figure $22 \mathrm{C}$ and Figure $23 \mathrm{C}$ ). Similarly, fits to the responses showed a sigmoid shape, comparable to what could be observed in controls (Figure $24 \mathrm{C}$ and Figure $25 \mathrm{C}$ ). Evaluating the $\Delta \mathrm{F} / \mathrm{F}_{0}$ gain per ${ }^{\circ} \mathrm{C}$ furthermore revealed no significant differences between the mutant strain and wildtype 
controls, in neither $\mathrm{HC}$ nor CC population (Figure 26).

Taken together, these results imply that NOMPC modulates the temperature evoked activity of the HCs in the Drosophila arista, and that NOMPC is necessary for the proper response amplitude of cells. Further the number of functional nompC gene copies seems to influence this modulation, hinting at a possible dosage dependency of NOMPC.

\subsubsection{NompC rescue restores $\mathrm{HC}$ response amplitude}

So far, I could show that mutations of nompC have a distinct effect on the $\mathrm{HCs} \mathrm{Ca}^{2+}$ response to temperature changes. To investigate whether a functional rescue of nompC ${ }^{3}$ null mutation could restore the function of the HCs. To this end, I used flies with a homozygous nomp $C^{3}$ mutation that expressed one copy of nompC under UAS control. I also controlled for effects of the UAS or Gal4 lines alone by measuring the response of flies with either construct in the homozygous nomp $C^{3}$ background.

The Gal4-UAS controlled expression of nompC was successful in restoring the response amplitude of the aristas HCs (Figure $22 \mathrm{~F}$ ) and CCs (23 F). Mean responses to relative temperature changes of arista HCs reached similar levels as the wildtype controls, and the response amplitude was clearly higher than in homozygous or heterozygous nompC ${ }^{3}$ mutants. In the same vein, functions fitted to the dataset of nompC-rescue showed a similar curve as the wildtype controls, including a sigmoid shape (Figure $24 \mathrm{~F}$ and Figure $25 \mathrm{~F}$ ). The effect of the rescue could be further confirmed when investigating the $\Delta \mathrm{F} / \mathrm{F}_{0}$ gain per ${ }^{\circ} \mathrm{C}$, which revealed no statistically significant difference between wildtype controls and the rescue line, while being significant in comparison to both homo- and heterozygous nompC $\mathrm{C}^{3}$ mutants (Figure $26 \mathrm{~A}$ ). While the effect of NOMPC on the CC signal appears to be rescued by the Gal4-UAS rescue as well, the gain per ${ }^{\circ} \mathrm{C}$ actually rises above the value of Cantons control flies, exhibting a significant difference (Figure $26 \mathrm{~B}$ ). UAS-nompC and nompC-Gal4 control flies in the homozygous nompC ${ }^{3}$ background responded similarly to the respective mutant background line and did not show a restoration of $\mathrm{HC}$ response amplitude. (No step data shown, as UAS and Gal4 controls were not exposed to all stimulus protocols; for $\mathrm{Ca}^{2+}$ traces, see Appendix, Figure A12).

\subsubsection{Overexpression of nompC does not significantly alter HC re- sponses}

The results described in 3.3.1 so far support the theory that the number of functional nompC gene copies might have a direct effect on the temperature evoked $\mathrm{HC}$ response. To further investigate this possible correlation, I assessed the temperature evoked $\mathrm{Ca}^{2+}$ re- 
sponses of flies expressing an additional nompC copy under UAS control. Expression was driven using the same nompC-Gal4 construct that was employed for the nompC rescue in 3.3.2. If the role of NOMPC in the HCs of the arista is one of a simple amplification of temperature triggered $\mathrm{Ca}^{2+}$, one might expect an increase in $\mathrm{HC}$ amplitude when introducing one more functional nompC copy into the HCs. Overexpression of nompC, however did not lead to an increase in $\mathrm{HC}$ response amplitude. While the mean response amplitude to relative temperature steps did not differ greatly from wildtype controls, there was a small trend towards lower responses in the $\mathrm{HC}$ population. (Figure $22 \mathrm{E}$ and Figure 23 E). Similarly, fits to the $\Delta F / F_{0}$ responses to relative temperature changes overlapped to a great extend with wildtype controls for both HCs and CCs (Figure $24 \mathrm{E}$ and Figure $25 \mathrm{E}$ ). Analyzing the $\Delta \mathrm{F} / \mathrm{F}_{0}$ gain per ${ }^{\circ} \mathrm{C}$ of the maximum responses furthermore reveals no significant effect of the overexpression in this measure (Figure 26). This observation could be due to a number of factors, such as postranslational regulation which might affect protein levels of the channel. The response amplitude of UAS and Gal4 lines alone did not differ greatly from the wildytype level (No step data shown, as UAS and Gal4 controls were not exposed to all stimulus protocols; for $\mathrm{Ca}^{2+}$ traces, see Appendix, Figure A12). These results indicate, that while a reduction of functional NOMPC leads to an impairment of $\mathrm{HC}$ function, which increases with the severity of the nompC knockout/knockdown, this effect is not simply scalable into the oppsite direction, as an increase in nompC copies did not alter $\mathrm{HC}$ responses significantly.

Taken together, the results from the physiological experiments suggest that the transduction mechanism of the arista HCs is a finely tuned system, in which NOMPC plays an important role in the regulation of temperature evoked $\mathrm{Ca}^{2+}$ influx, and alterations to this system in, which the amount of functional nompC genes is reduced, impairs the overall sensitivity of the $\mathrm{HC}$ population to temperature changes. For an overview visualization of the described effects of nompC on the arista neuron responses, the gain of a linear fit to the $\Delta F / F_{0}$ responses of all tested Drosophila strains to relative temperature changes is shown in Figure 27. 


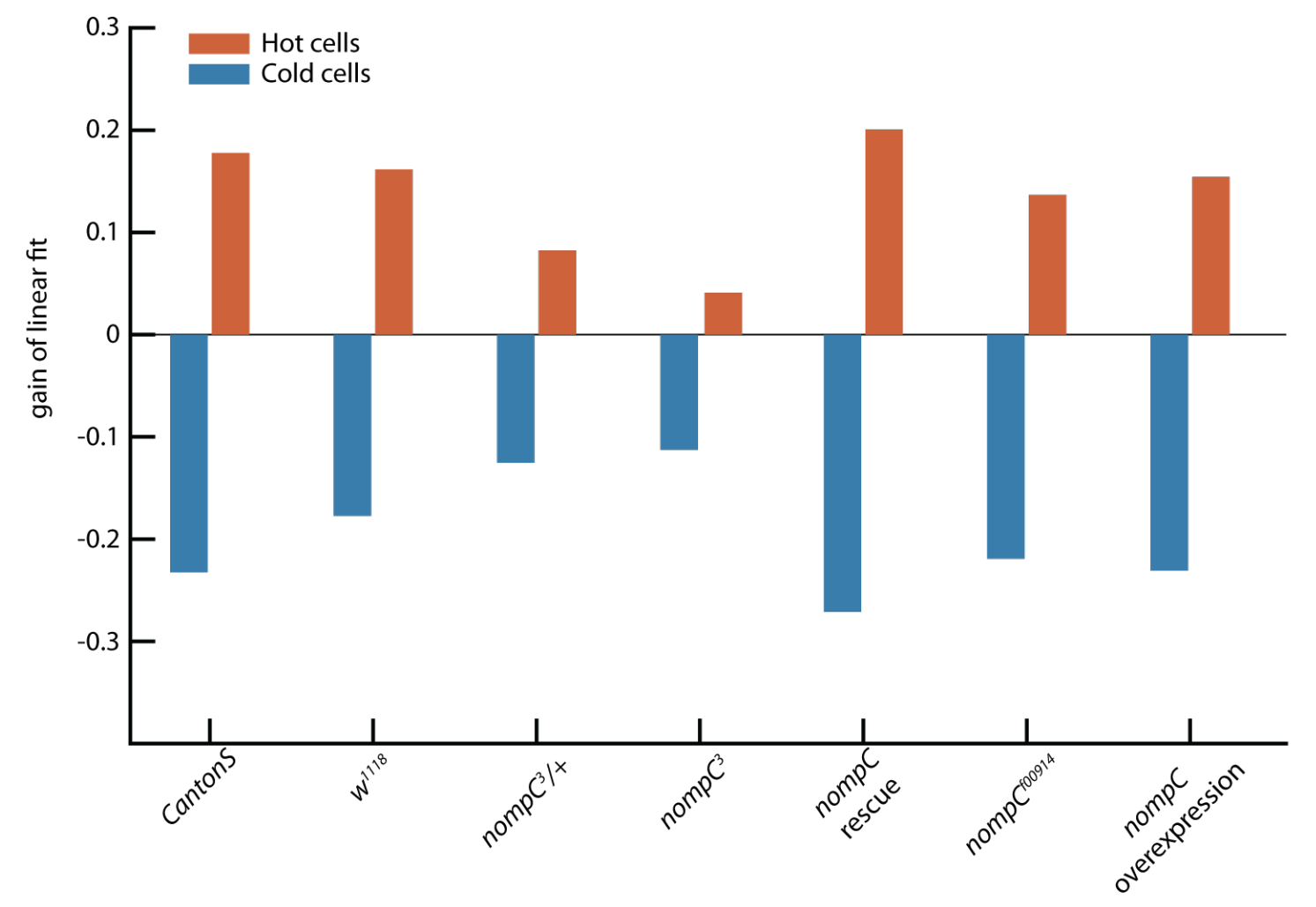

Figure 27: Gain of linear fits to hot and cold cell responses $F$ its to the $\Delta F / F_{0}$ response values of the tested fly strains in temperature step experiments. Mutants of nompC exhibit considerable reduction in $\Delta F / F_{0}$ per degree $\mathrm{C}$ in $\mathrm{HCs}$, which appears to become stronger with the severity of the mutation. While CC response appears to be affected as well, the effect did not appear to be as strong as in the HC population, and the CC response amplitude of nompC $\mathrm{C}^{\mathrm{f00914}}$ mutants was not reduced compared to wildtype flies. Overexpression of nompC did not appear to strongly alter $\mathrm{HC}$ or $\mathrm{CC}$ responses.

\subsection{Arista neurons do not show calcium responses to me- chanical deflection}

So far, the neurons of the arista have been mainly studied in the context temperature sensation. Since NOMPC has been shown to be a mechanosensitive ion channel (Cheng et al. 2010; Effertz, Wiek, and Göpfert 2011; Göpfert, Albert, et al. 2006; Walker, Willingham, and Zuker 2000; Yan et al. 2013; Zhang, Yan, et al. 2013), a question that presents itself is whether the nompC expressing $\mathrm{HCs}$ of the arista show activity in response to mechanical deflection. Therefore, I deflected the arista using a piezo actuator and recorded the change in fluorescence from the calcium indicator GCaMP6m. The antenna of the flies were fixated, to that mechanical deflection of the arista would only bend it and not rotate the funiculus or move other part of the antennae. After mechanical stimulation, a hot and cold temperature step was used to identify $\mathrm{HC}$ and CC populations (see 2.4.2 for 
details).

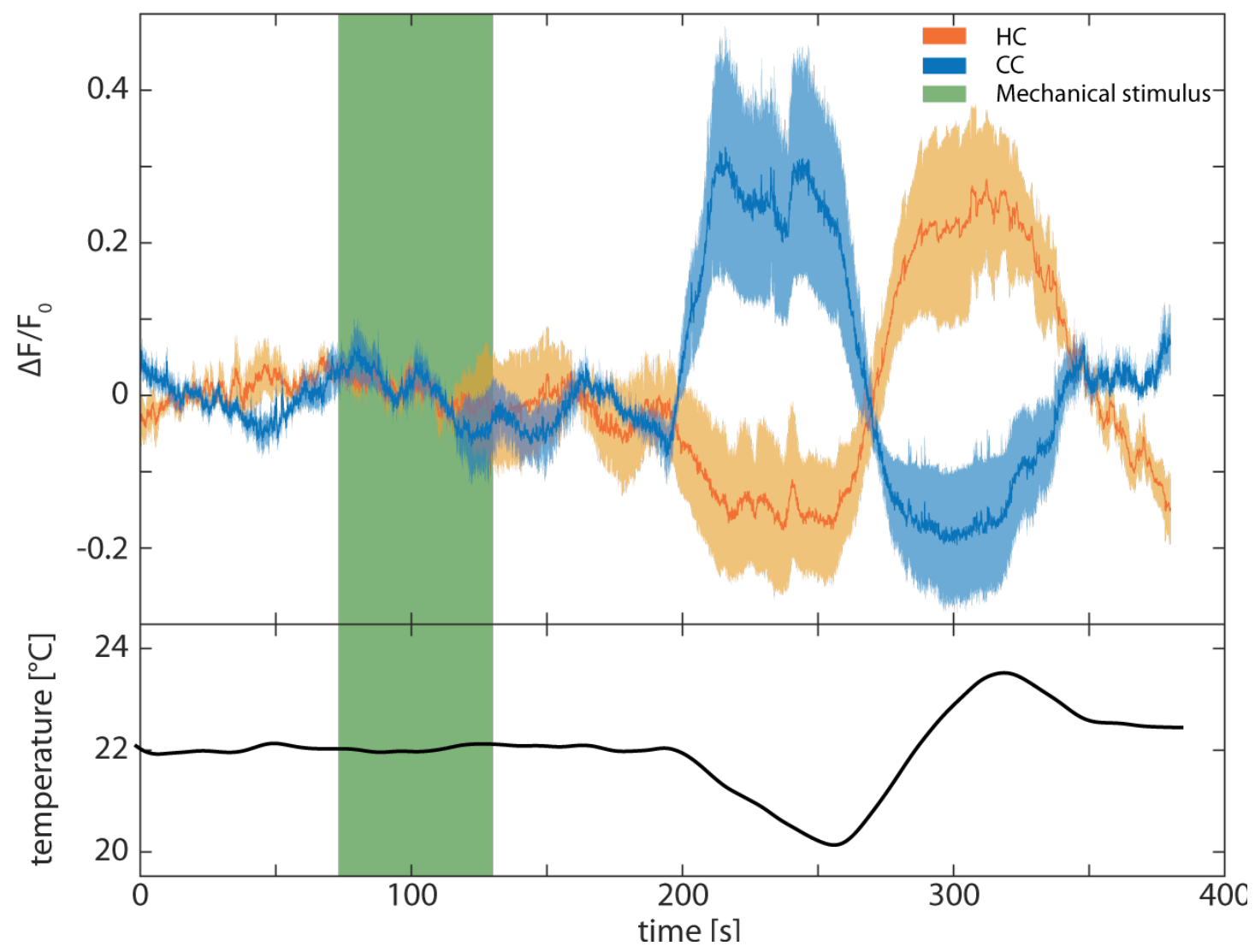

Figure 28: Calcium responses to mechanical stimulation of the arista $\Delta F / F_{0}$ signal of arista neurons in $w^{1118}$ control flies during mechanical stimulation. Solid lines denote mean responses, shaded areas the SEM. HC responses shown in orange, CC responses in blue. Period of mechanical deflection of the arista is marked in green. After a initial waiting period of $75 \mathrm{~s}$, the arista was deflected for $60 \mathrm{~s}$ using a bend glass capillary attached to a piezo actuator. After mechanical stimulation, a 60 s pause was held before a 60 s cold step $\left(20^{\circ} \mathrm{C}\right)$ and a 60 s hot $\left(24^{\circ} \mathrm{C}\right)$ temperature step were applied to identify $\mathrm{HC}$ and $\mathrm{CC}$ populations. No distinct change in fluorescence could be observed during the mechanical stimulation/bending of the arista in either group of neurons. Temperature evoked responses were still functional. This implies no mechanosensitivity of the arista neurons.

I could not observe responses triggered by the mechanical stimulation (Figure 28). A constant deflection for $60 \mathrm{~s}$ did not lead to any observable change in $\Delta F / F_{0}$. Response of both $\mathrm{HC}$ and $\mathrm{CC}$ populations towards temperature increase and decrease was still functional. These experiments therefore imply that the arista neurons are not mechanosensitive, and even the expression of nompC, which has been shown to be mechanotransducer channel, does not render the HCs mechanosensitive. 


\subsection{NOMPC affects temperature preference and tolerance behaviour}

After establishing that NOMPC modulates temperature evoked activity of the HCs in the arista, a question to consider is whether this influence extends to the behaviour. When observing the physiological data from the experiments described above, it can be hypothesized that NOMPC affects the behaviour towards hot temperatures. Actual behaviour, however, is of course the ultimate output of an animal system and influenced by a combination of all sensory inputs and internal states. It is therefore not obvious, if the effect of nompC mutations on HCs (altering, but not abolishing the responses) also has an influence on the behavioural level. Even more so, as Drosophila possesses additional temperature sensitive neurons (see 1.3.1). Thus, the effect of nompC mutations and overexpression on the temperature preference and avoidance behaviour of adult Drosophila was assessed by tracing and evaluating the movement of adult flies within a temperature gradient, as described in 2.5.

I compared the temperature preference and avoidance behaviour of nompC $\mathrm{foog}^{\mathrm{H}}$ mutants, heterozygous nomp $\mathrm{C}^{3}$ mutants and nompC overexpressing flies to wildtype controls in the CantonS and $w^{1118}$ background. HC-Gal4, UAS-hid,rpr served as control group for flies with abolished heat sensation in the arista, as the HC population in these flies is ablated using apoptotic factors Hid and Reaper (Grether et al. 1995; White et al. 1994) under UAS control, and driven specifically in the HCs via the Gr28b.d-Gal4 construct (herein refered to as HC-Gal4) (data for the HC-Gal4, UAS-hid,rpr flies was kindly provided by Dr. D. Giraldo). It was not possible to employ homozygous nompC ${ }^{3}$ mutants, or flies with a similarly strong knockdown of nompC, as the severe impairment of mechanosensation and locomotion means these Drosophila do not show any coordinated locomotion behaviour, thus preventing the use in any form of locomotion based experiment. Flies with the UAS controlled rescue of nompC, which have been used in the physiological experiments (See 3.3), were employed in these behavioural experiments, as they again show proper locomotion behaviour, and I could therefore assess their temperature driven behaviour, compared to wildtype controls and heterozygous nomp ${ }^{3}$ mutants. The distributions of all fly strains were corrected for cold-trapping, using IGLOO (Giraldo et al. 2019), as described in 2.5.2.

Flies of the Cantons strain showed a peak distribution in the range between $23-24^{\circ} \mathrm{C}$ (Figure 29A) and a median preferred temperature of $21.5^{\circ} \mathrm{C}$ (Figure $30 \mathrm{D}$ ). Temperatures below $15.5^{\circ} \mathrm{C}$ and above $26.5^{\circ} \mathrm{C}$ were actively avoided (Figure $30 \mathrm{~A}-\mathrm{B}$ ). $\mathrm{W}^{1118}$ flies showed a similar preference behaviour, with a median preferred temperature of $21.5^{\circ} \mathrm{C}$ (Figure 30 A), while avoiding temperatures below $15.5^{\circ} \mathrm{C}$ and above $26^{\circ} \mathrm{C}$ (Figure $30 \mathrm{~A}-\mathrm{B}$ ). The density distribution along the temperature gradient shows a peak between $18-20^{\circ} \mathrm{C}$ (Figure 
A

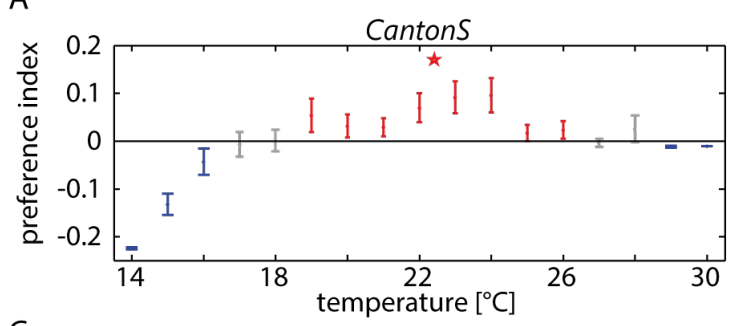

C

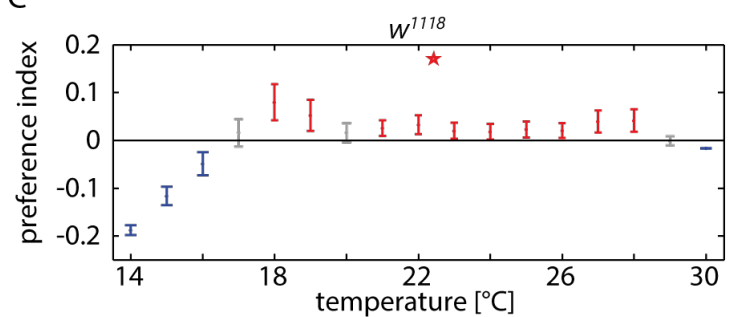

$E$

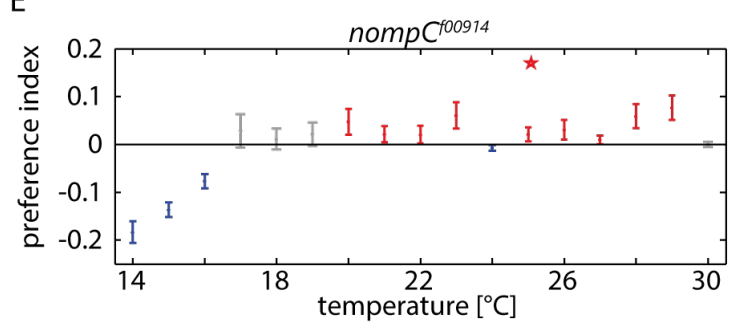

G

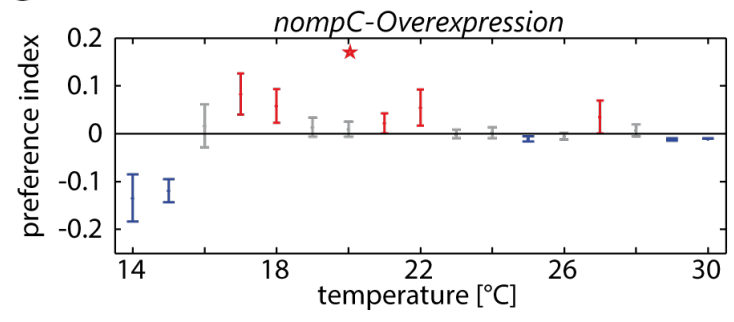

B

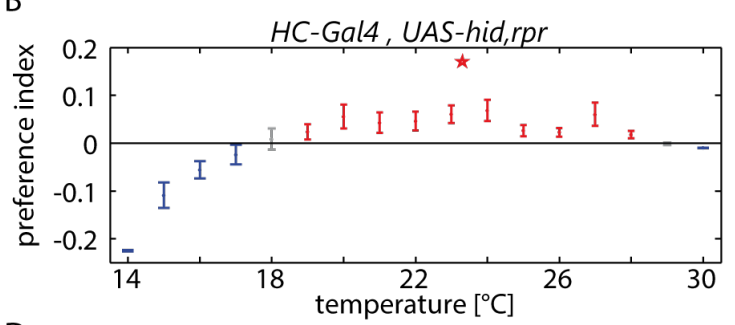

D

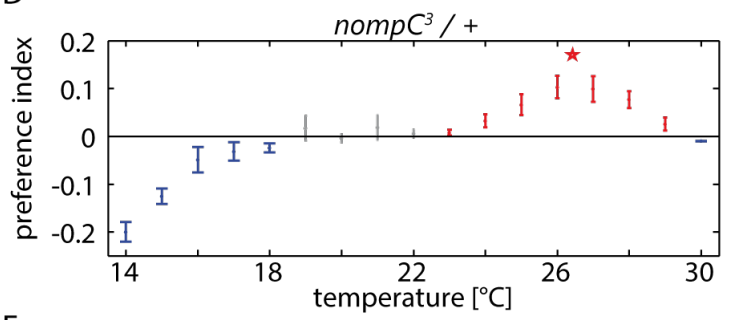

$\mathrm{F}$

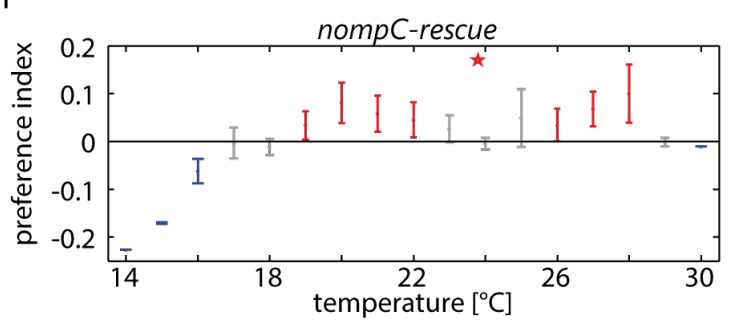

preferred
antipreferred
no preference

Figure 29: Temperature distributions of Drosophila Histograms show the grouped distribution of the different fly lines along the temperature gradient in behavioural experiments, after correction of cold bias via IGLOO. Error bars denote $95 \%$ confidence intervals. Preferred temperatures are shown in red, avoided (antipreferred) temperatures in blue, and grey denotes temperatures which are neither preferred nor avoided. Mean preferred temperature is marked with a red star. A), C) Distributions of control strains show similiar mean preference for flies in CantonS and $w^{1118}$ B) Flies with ablated hotcells exhibit a visible shit of mean temperature preference to hotter temperatures and a wider range of preferred temperatures at the hot side. D), E) nompC $\mathrm{f}^{\mathrm{f0}}{ }^{\mathrm{s}}$ and heterozygous nomp $\mathrm{C}^{3}$ animals exhibit a hot-shift in mean temperature preference and a range of preferred temperatures that continues farther into the hot range, when compared to wildtype controls. G) nompC-rescue flies still exhibit an effect when compared to wildtype flies, but not as severe as in the mutants. nompC overexpression leads to lower preferred temperature and a wider range of temperatures that are neither preferred nor avoided.

n: CantonS: 45, w ${ }^{1118}:$ 50, HC-Gal4, UAS-hid,rpr: 40, nompC $3 /+$ : 35, nompC foo914: 52, nompC overexpression: 29 , nompC rescue: 16. 
29B).

Behavioural data in the temperature gradient for Drosophila with ablated HCs (HC-Gal4, UAS-hid,rpr) revealed a distinct phenotype, when compared to the wildtype controls. Median preferred temperature was found to be at $23.5^{\circ} \mathrm{C}$, about $2^{\circ} \mathrm{C}$ higher than the CantonS and $w^{1118}$ lines, although not a significant increase (Figure $30 \mathrm{D}$ ). Avoidance of cold temperatures was found in range very similar to the wildtype controls, starting approximately below $15.5^{\circ} \mathrm{C}$ (Figure $30 \mathrm{~A}$ ). Hot temperature avoidance on the other hand was found to only begin at a median temperature above $28^{\circ} \mathrm{C}$, a significant increase when compared to CantonS (Figure $30 \mathrm{~B}$ ). As would be expected, loss of the $\mathrm{HC}$ population of arista neurons appears to result in a strong reduction in the avoidance of hotter temperatures, while cold avoidance and median temperature preference are not strongly affected.

Assessing the behaviour of heterozygous nompC $C^{3}$ mutant flies in the temperature gradient experiment revealed effects on temperature preference and avoidance: Distribution density was shifted much more towards the hotter end of the temperature gradient, with the peak of distribution being around $26-27^{\circ} \mathrm{C}$ (Figure $29 \mathrm{D}$ ). Consistent with the effect observed in the $\mathrm{Ca}^{2+}$ imaging experiment described in 3.3, the mutant flies showed a significant increase in both the start of hot avoidance (towards $27^{\circ} \mathrm{C}$ ) and median preferred temperature (shifting towards $25^{\circ} \mathrm{C}$ ) when compared to wildtype controls (Figure $30 \mathrm{~B}, \mathrm{D}$ ) or the aforementioned hid-rpr construct. The onset of cold avoidance behaviour meanwhile was not significantly affected. (Figure $30 \mathrm{~A}$ ).

Flies of the nompC rescue strain, carrying a homozygous nomp $\mathrm{C}^{3}$ mutation and a functional copy of the gene under UAS control, could restore the temperature driven behaviour of Drosophila to wildtype levels: While a slight shift in the distribution along the temperature gradient was still observable (Figure $29 \mathrm{~F}$ ) neither cold avoidance (at $15.5^{\circ} \mathrm{C}$,) nor hot avoidance (at around $26.5^{\circ} \mathrm{C}$ ) or even median preferred temperature $\left(22^{\circ} \mathrm{C}\right.$ ) were distinctly different from wildtype lines (Figure 30 A, B, D). Start of hot avoidance and preferred temperature, were furthermore significantly lower than in the heterozygous nompC $C^{3}$ line. Even though the nompC rescue line did not restore the hot cell response amplitude to wildtype completely in the $\mathrm{Ca}^{2+}$ imaging experiments, the effect appears to be sufficient to rescue temperature driven behaviour.

Nomp $\mathrm{C}^{\mathrm{f00914}}$ mutant flies on the other hand still exhibited a shift in the distribution density towards hotter temperatures and a shift in the mean temperature preference of the distribution, but no clear peak in the distribution could be observed, with the highest preference indices lying at 29 and $23^{\circ} \mathrm{C}$ (Figure $29 \mathrm{E}$ ). Furthermore, comparing the start of cold and hot avoidance, or the median preferred temperature to the wildtype control lines, reveals no significant difference (Figure $30 \mathrm{~A}, \mathrm{~B}, \mathrm{D}$ ), in contrast to the effect of the heterozygous nompC $\mathrm{C}^{3}$ mutation described above.

Lastly the effect of the expression of an additional nompC copy under UAS control in a 
wildytpe background on the temperature behaviour was analyzed. NompC overexpression lead to overall flatter distribution along the gradient, with preference index peaking at around 22 and $17^{\circ} \mathrm{C}$ (Figure $29 \mathrm{G}$ ). Of the strains tested in this study, only the nompC overexpression lead to and significantly lower onset in the start of cold avoidance, at a median of around $14.75^{\circ} \mathrm{C}$, and an earlier start of hot avoidance, at approximately $24.5^{\circ} \mathrm{C}$ (Figure $30 \mathrm{~A}-\mathrm{B}$ ). Median preferred temperature was also found to be significantly lower than in all other tested strains, at $18^{\circ} \mathrm{C}$ (Figure $30 \mathrm{D}$ ).

We define a tolerated temperature as those temperatures that are not avoided. The range of these tolerated temperatures (as in how many different temperatures are tolerated) is also significantly higher in heterozygous nompC ${ }^{3}$ than in wildytpe control lines and the nompC rescue line, but not higher than in the HC-Gal4, UAS-hid,rpr flies (Figure $30 \mathrm{C})$. This line with ablated HCs shows a significantly larger range of tolerated temperatures than CantonS flies. Range in tolerated temperatures was also significantly increased in the nompC overexpression when compared to CantonS, while nompC $C^{60914}$ and nompC rescue lines showed no significant differences compared to wildtype flies (Figure $30 \mathrm{C}$ ).

To summarize, NOMPC appears to affect temperature driven locomotion behaviour of adult Drosophila: Heterozygous nompC $\mathrm{C}^{3}$ mutant flies exhibited a significant shift in the onset of heat avoidance towards hotter temperatures, as well as a significantly higher preferred temperature compared to wildtype control flies. Mutations of nompC did not have a significant effect on cold avoidance, although flies overexpressing nompC showed a somewhat reduced cold avoidance. Rescue of nompC $C_{3}$ did restore the behaviour back to wildtype levels. 
A

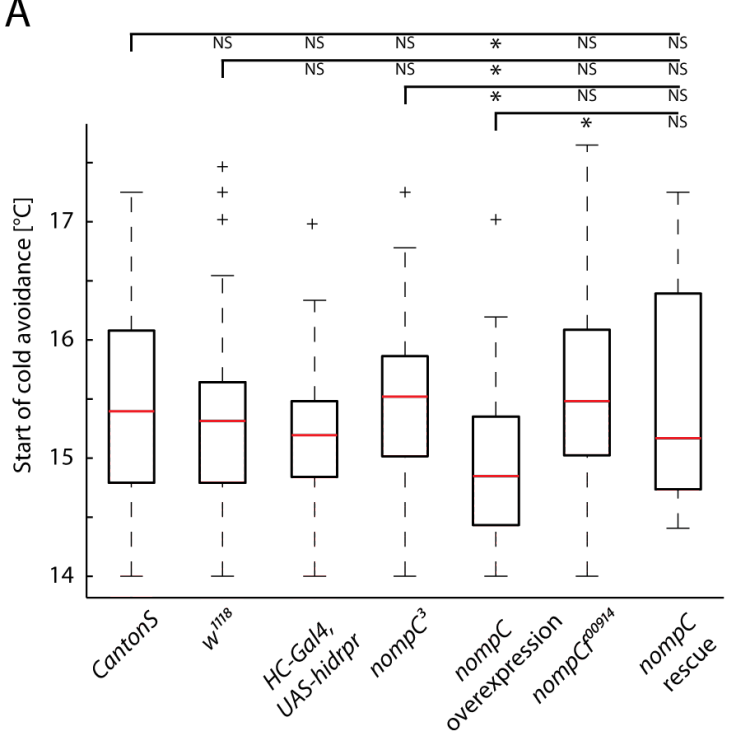

C

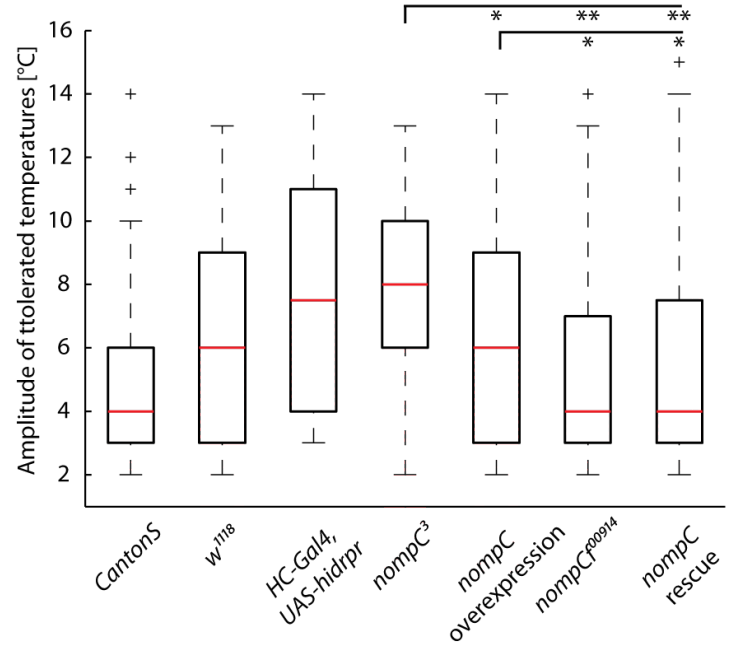

B

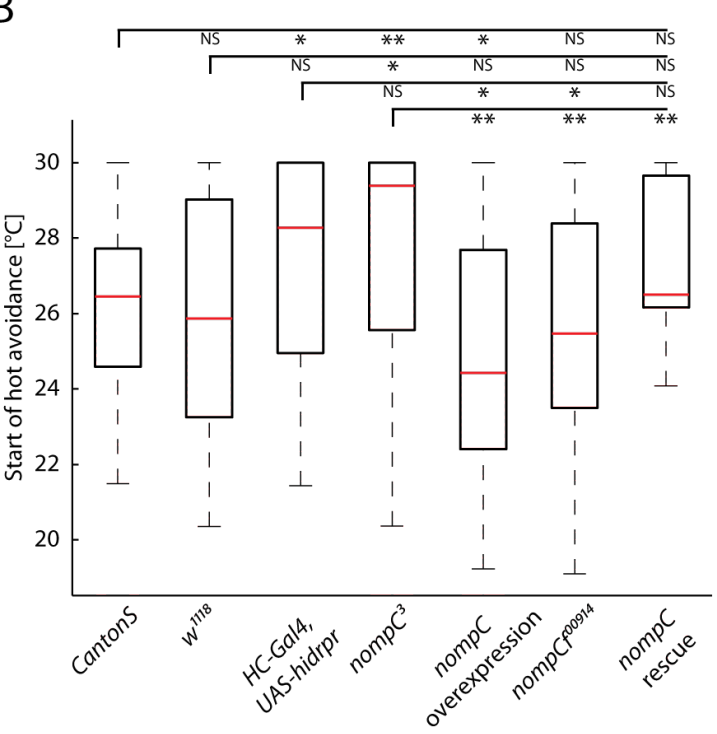

D

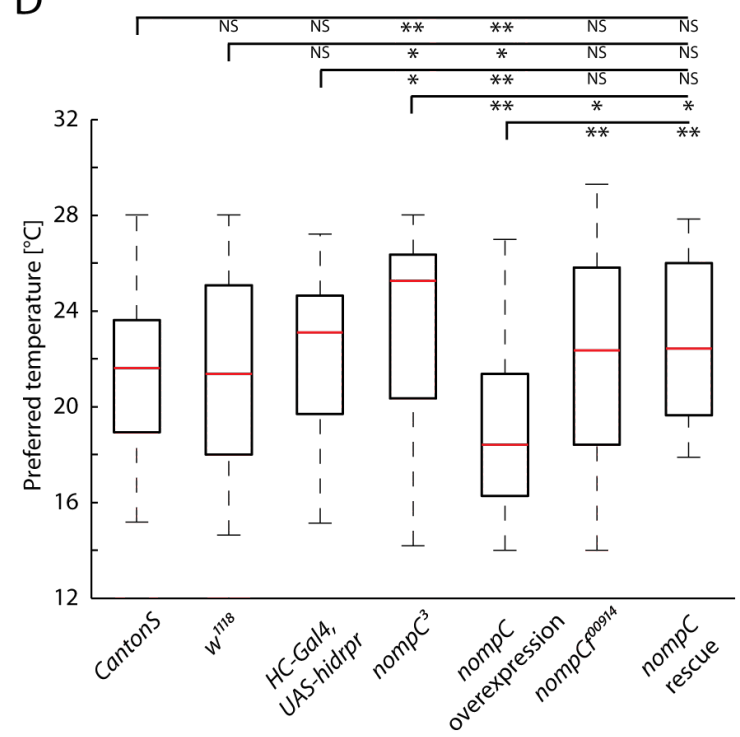

Figure 30: Comparison of temperature avoidance and preferences Red lines indicate median values, boxplots generated as described in 2.7 (page 31). Significances as: ${ }^{*}=$ $\mathrm{p}<0.05 ;{ }^{* *}=\mathrm{p}<0.01 ;{ }^{* * *}=\mathrm{p}<0.001$; NS: not significant. Not shown comparisons are not siginificantly different. A) Start of cold avoidance. Flies carrying the nompC overexpression are the only line exhibiting a significant difference, with cold avoidance starting at siginificantly lower temperatures than in controls. B) Start of hot avoidance. Drosophila with ablated HC population ( $\mathrm{HC}$-Gal4, UAS-hid,rpr) exhibit a later start of hot avoidance when compared to CantonS controls. An even stronger shift towards later hot avoidance can be observed in heterozygous nompC ${ }^{3}$ mutant flies, with the effect being rescued in the nompC rescue line, which exhibits no significant difference compared to wildtype controls. Overexpression of nompC appears to lead to an earlier start of hot avoidance on the other hand. C) Amplitude of tolerated temperatures. Heterozygous nomp $\mathrm{C}^{3}$ mutations, nompC overexpression and ablation of HCs leads to a wider range of preferred temperatures. D) Preferred temperatures. Heterozygous nomp $\mathrm{C}^{3}$ mutant flies show a significantly higher preferred temperature than all other tested fly lines. Overexpression of nompC on the other hand leads to a significantly lower preferred temperature. 


\section{Chapter 4}

\section{Discussion}

Accurate sensing of ambient temperatures is an essential ability for all behaving animals and the study of temperature sensation has become a considerable body of research in the field of sensory neuroscience. On Earth alone extreme temperature ranges are found, with highest temperatures at underwater hydrothermic vents at over $400^{\circ} \mathrm{C}$ and lowest temperatures about $-100^{\circ} \mathrm{C}$ in antarctic ridges and even those extremes are habtitats to extremophiles. With respect to the wide range of uninhabitable temperatures on our planet, it becomes clear that thermoregulation is necessary for survival. Even though that the tolerable range for Drosophila spans more than twenty degrees it is a question of survival and fitness for the tiny insect to avoid harmful temperatures.

Many recent studies have taken advantage of the available genetic, physiological and behavioural tools available for the fruit fly, Drosophila melanogaster, to uncover and understand the molecular and cellular mechanisms behind thermosensation (Barbagallo and Garrity 2015; Budelli et al. 2019; Fowler and Montell 2013; Gallio et al. 2011; Ni, Bronk, et al. 2013; Tracey Jr et al. 2003). However, many aspects of the transduction and regulation processes of thermosensation in Drosophila remain unknown. Here, I have presented evidence that a set of temperature sensing neurons in the arista of Drosophila functions as relative sensors for temperature changes, rather possessing an activation threshold of an absolute temperature. Furthermore, I collected data which suggests that the mechanotransducer channel NOMPC (a member of TRP family of ion channels) is involved in signal transduction in these arista neurons, specifically in the heat sensing sub-set of cells and is not only required for proper responses of the neurons, but also for temperature preference and avoidance behaviour. 


\subsection{The response properties of thermosensitive arista neu- rons}

\subsubsection{Arista neurons are relative temperature sensors}

A total of six temperature sensitive neurons have been identified in the arista of Drosophila, three of which show excitatory responses to temperature increments, and are therefore referred to as hot cells $(\mathrm{HC})$ and three which exhibit excitatory responses to temperature decrements, called cold cells (CC) (Barbagallo and Garrity 2015; Foelix, Stocker, and Steinbrecht 1989; Gallio et al. 2011; Sayeed and Benzer 1996). In this study, I present evidence that the temperature evoked $\mathrm{Ca}^{2+}$ responses of both $\mathrm{HCs}$ and CCs differ in amplitude, depending on the temperature stimulus. Response amplitude in this context appears to be mainly determined by the relative, rather than absolute change in temperature: Temperature stimuli of the same relative change, but at different absolute temperature levels did not elicit siginificantly different responses, while temperature stimuli that reached the same absolute value but with a different relative change did result in different response amplitudes (See 3.2, starting at page 36).

Earlier research on the thermosensitive neurons in the arista, performed by $\mathrm{Ni}$ et al. (Ni, Bronk, et al. 2013) suggested that the HC and CC structures might be less involved in the determination of a preferred temperature for the animal and more of a sensor set for the detection of short term temperature change in the environment. This appears to be supported by the morphological situation of the temperature sensors: An external thermosensor should be located most distal to the body and by an appendage of small diameter to avoid being biased by the core temperature of the organism. The response properties that were observed in this study fall into line with these suggestions, as quick and precise assessment of temperature changes in the environment provide a relevant feedback for the animal when exploring its environment. Other thermosensitive structures in the fly have been implied in the determination of preferred temperatures, such as dTRPA1 positive neurons that are responsible for the response to more sustained temperatures that reach the inner body of the animal (see for example Hamada et al. 2008; Tang et al. 2013). When these sensors provide information about whether the fly is experiencing temperatures around its preferred range or not, it would make sense to rely on an external relative sensor during locomotion to gauge whether the animal is leaving a certain temperature range and how stark the contrast is, without a need for immediate information about the absolute temperatures. This would allow, for example for more rapid avoidance behaviour towards unfavorable temperature changes, which is especially important for a small poikilothermic animal (Heinrich 2013; Sayeed and Benzer 1996).

The idea that the arista neurons are relative temperature sensors also coincides with 
very recent studies by Budelli et al., which analyzed the spiking rate of arista neurons and stated that the HCs and CCs would act more akin to "heating" and "cooling" cells (Budelli et al. 2019). However, one has to keep in mind that the exact way by which information from different temperature sensitive structures (and even other sensory modalities for that matter) is integrated in higher order brain regions is still subject to ongoing research. In this context, it has been found that the information of the arista neurons, in addition to reaching the PAL region via the projections, might be relayed to central brain areas involved in learning and determination of temperature preference, such as the mushroom body (Frank, Jouandet, et al. 2015; Liu, Mazor, and Wilson 2015, early anatomical evidence found in Stocker et al. 1990, for a review see Florence and Reiser 2015). It is therefore not likely that the arista neurons can be excluded from a complex mechanism like determining and finding a preferred temperature of the organism, even if there primary role appears to be that of external temperature change sensors.

\subsubsection{Arista neurons show phasic-tonic responses to prolonged temper- ature changes}

To study potential adaptation processes in the temperature evoked responses of $\mathrm{HCs}$ and CCs, I employed experiments in which the animal was exposed to a constant temperature step (either cold or hot) for a total of 5 minutes, before returning to the starting temperature. Interestingly, while an inital phasic peak in the $\Delta \mathrm{F} / \mathrm{F}_{0}$ signal could be observed in the exciatory responses of both $\mathrm{HC}$ and CC populations, the signal did not adapt back to the starting niveau. This is reflected in the large $\tau$ values of exponential decay functions at 163 and 407 seconds for $\mathrm{HC}$ and CC respectively, suggesting a considerable tonic component in the excitatory temperature responses. This effect was even more pronounced when observing the inhibitory responses to the "non-preferred" stimulus direction of both cells (meaning cooling for $\mathrm{HCs}$ and heating for $\mathrm{CCs}$ ):The inhibitory $\mathrm{Ca}^{2+}$ responses did not exhibit any observable initial phasic response peak (Figure 21, page 44).

These findings could imply that both cell types, in addition to their proposed role as relative temperature change sensors, might actually also obtain to a certain degree information about absolute temperature values. These findings are complemented by very recent data from Budelli et al., who emplyed electrophysiological recordings of the arista neurons and found that HCs exhibited both phasic increases in spiking rate after temperature increases, as well as a tonic component, in which the general spiking was to a considerable amount influenced by the external temperature level when held at a steady level for extended periods of time (Budelli et al. 2019). Intriguingly, Budelli et al. did report that no tonic responses could observed in the CC population of arista neurons, findings only fast adapting, phasic changes in spiking rate to temperature changes. While these findings do not overlap with the observed $\mathrm{Ca}^{2+}$ responses of CCs in this study, I 
cannot exclude a possible role of the temperature stimulus in these observations, as the stimuli employed in this study consisted of continuous changes which usually took up to a minute to reach the target temperature, while Budelli et al. employed much sharper stimuli, with temperature changes of $5^{\circ} \mathrm{C}$ in less than 10 s. Additionally, one has to keep in mind that electrophysiological recordings and $\mathrm{Ca}^{2+}$ imaging are different measures of neuronal activity and findings from one method might not be directly transferable into the other measure. The intrinsic properties of $\mathrm{Ca}^{2+}$ imaging must also be considered when observing time based processes, such as adaptation, as ion diffusion/transport across the membrane and binding towards reporter proteins, like the here used GCaMP6m, have intrinsic time constants that must be kept in mind (Chen et al. 2013). This might be a deciding factor why for example the majority of adaptation after peak response observed by Budelli et al. happens within 30s, while the process was taking up to a minute in the here performed $\mathrm{Ca}^{2+}$ imaging experiments.

After the temperature stimulus in the adaptation experiment ended and temperature was returned to the starting value of $22^{\circ} \mathrm{C}$, both $\mathrm{HCs}$ and CCs $\Delta \mathrm{F} / \mathrm{F}_{0}$ did not simply return to base level, but showed an "overshoot" (Figure 21, page 44). This again illustrates the function of the arista neurons as relative sensors, as the return to the starting temperature would not just be considered as the end of an absolute temperature step, but would be perceived as a relative change in temperature, opposite in direction to the temperature change experienced at the beginning of the stimulus.

To summarize, the findings of the first part of this thesis shed more light on the temperature responses by thermoreceptors of the arista. Complementary to the cold cell analysis of Budelli et al., I could characterise the responses of the so far elusive hot cells. In Budelli et al. HC responses could only be analysed after ablating CCs (Budelli et al. 2019). The thermosensitive neurons in the arista of Drosophila respond to relative temperature change, with $\mathrm{Ca}^{2+}$ response amplitude increasing with stronger changes in temperature. Adaptation experiments furthermore imply that in addition to this relative sensor role, both $\mathrm{HCs}$ and $\mathrm{CCs}$ exhibit tonic response component to prolonged temperature stimulation.

\subsection{NOMPC modulates hot cell responses}

Research in recent years has begun to gather comprehensive information on the molecular basics of thermosensation in the arista neurons. A number of proteins have been shown to be involved in the temperature repsonses of the $\mathrm{CC}$ population, although there has yet to be a concise explanation on how the different members of the ionotropic receptor (IR) and Brivido (Brv) families work and possibly interact (Budelli et al. 2019; Gallio et al. 2011). In the context of the warming responsive HCs, only one protein has been proven 
to be essential in the transduction process so far, the gustatory receptor GR28B(D) (Ni, Bronk, et al. 2013). Data from the $\mathrm{Ca}^{2+}$ experiments performed in this study shows a considerable effect of the TRP channel NOMPC on the the temperature evoked responses of the arista neurons (see 3.3, starting at page 45). Consistent with the findings that nompC is expressed in the $\mathrm{HC}$ population of arista neurons, the effect of nompC mutations appeared to take a great effect on the response amplitudes of these cells. Flies carrying the null mutation nomp $\mathrm{C}^{3}$ exhibited severe reductions in $\mathrm{HC}$ response amplitudes compared to wildtypes, while flies that were heterozygous for the mutation showed a more intermediate phenotype, with amplitudes above those of homozygous mutants but below wildtype level. The mutant phenotype could be rescued, using one wildtype copy of the gene under UAS control. The hypomorphic nomp $C^{\text {f00914 }}$ allele (see for example Sun et al. 2009) led to a reduction in response amplitude in the range of moderate temperature increases, from +3 to $+6^{\circ} \mathrm{C}$. Interestingly, the effect of nompC mutations was not exclusive to the HCs. While the expression analysis suggests a localisation specific to the $\mathrm{HCs}$, the response amplitude of CCs was affected as well, albeit not as strongly as that of the HCs. How the mutation of a protein in one cell group might affect the other is not immediately clear, as no direct interaction or processes similar to lateral inhibition have been described in the arista so far. The neurons of the arista are organized into distinct sensilla, consisting of 2 neuronal dendrites (one HC and one CC dendrite), enveloped by two sheath cells (Foelix, Stocker, and Steinbrecht 1989). This close anatomical pairing of $\mathrm{HC}$ and $\mathrm{CC}$ neurons could make a cell to cell interaction at the level of the sensory neuron possible. However, anatomical studies have so far not found a clear indication of cell to cell contacts or similar structures, making the implications that a protein of one cell type might influence the other quite surprising.

NOMPC has been shown to be a mechanotransducing ion channel (Walker, Willingham, and Zuker 2000; Yan et al. 2013), necessary for processes such as hearing, touch sensation and propioception in Drosophila (Cheng et al. 2010; Effertz, Wiek, and Göpfert 2011; Göpfert and Robert 2003). This of course raises the question on the role of the mechano TRP in the temperature sensing neurons of the arista. NOMPC itself has not been implied to be temperature sensitive. Furthermore, during the $\mathrm{Ca}^{2+}$ imaging experiments of this project, no distinct fluorescence change from the Johnston's organ (which, in its role as the fly's hearing organ, houses around 500 nompC expressing neurons (see for example Effertz, Wiek, and Göpfert 2011; Todi, Sharma, and Eberl 2004)) could be observed. The modulatory effect of NOMPC is therefore more likely to originate from the interaction with other factors in the thermotransduction pathway or the regulation of the transduction process.

The fact that an ion channel acts in a modality outside of its described canonical main function or interacts with other transduction mechanisms/channels is not unprecedented and comparable examples have been found in a range of neuronal systems and 
animals. A immediate comparison that comes to mind in this context is the mammalian ion channel TREK-1. Like the TRP channel NOMPC that was the subject of this study, TREK1 has been shown to play a role in both mechano- and thermosensation (Maingret, Lauritzen, et al. 2000). TREK-1 is a $K_{2 p}$, a two pore potassium channel, a group that plays a role in regulation cell excitability via rectifying $\mathrm{K}^{+}$efflux (for review, see Enyedi and Czirják 2010). However, in contrast to NOMPC, TREK-1 has been proposed to be gated by mechanical forces and heat (Brohawn, Su, and MacKinnon 2014; Chemin et al. 2005; Maingret, Lauritzen, et al. 2000; Noël et al. 2009; Plant 2012; Schneider et al. 2014), whereas no intrinsic gating via temperature has been found for NOMPC. So while the general mechanism and effect differ between the two channels, it does serve as an example for ion channels that play an active role in the transduction process of both mechanoand temperaturesensation.

The exact mechanism by which NOMPC modulates the HCs in the arista remains speculative. So far, only one other transducer channel has been shown to function in the HCs: the gustatory receptor GR28B(D) (Ni, Bronk, et al. 2013). A knockout of GR28B(D) abolishes temperature induced activity of the HC population (Ni, Bronk, et al. 2013) and misexpression in cells that are normally not temperature sensitive confers heat-sensitivity (Mishra et al. 2018). It is reasonable to assume that this gustatory receptor serves the role of the primary temperature transducer in the HCs and NOMPC acts in a modulatory fashion. It is well known that ion channels can shape the response properties of other transducer channels. An effect in this manner has for example been described for NOMPC and its interaction with Brv1 in the gentle touch sensation of Drosophila larvae, in which Brv1 appears to amplify/shape the mechanically induced response of NOMPC (Zhang, Li, et al. 2018). Experiments have shown that the conductance for $\mathrm{Ca}^{2+}$ ions of GR28B(D) is comparatively low (Mishra et al. 2018). It thus seems possible that while GR28B(D) is responsible for the initial response of the cell towards temperature changes, a considerable portion of the actual ion conductance across the membrane is contributed by the NOMPC TRP channel. This theory of NOMPC acting as a secondary mediator of ion conductance might also provide a possible explanation of the observed data, implying a gene dosage effect: if a heteozygous nomp $\mathrm{C}^{3}$ mutation leads to a reduction of functional NOMPC protein amount, it could provide less ion conductance across the membrane. This effect would be even more severe in homozygous mutants, which reportedly exhibit no detectable amount of NOMPC anymore (Liang et al. 2011), coinciding well with the observed experimental data from $\mathrm{Ca}^{2+}$ experiments.

Overexpressing nompC in the HCs by introducing an additional gene copy under UAS control did not alter the response properties of the arista neurons significantly. This could be due to different reasons: As I did not employ electrophysiological recordings, I cannot make assumptions about the actual membrane potential during the temperature experiments, and it might be possible that in wildtype flies, responses to big temperature 
steps that lead to the opening of NOMPC already approach the reversal potential of certain ions. This could imply that further NOMPC channels do not alter the ion transport across the membrane. I also cannot exclude that regulatory factors within the arista cells compensate for the additional gene copy of nompC. Cells show robust mechanisms to regulate the amount of proteins which are critical to the function of the cell, sometimes referred to as gene dosage compensation (see for example El-Brolosy and Stainier 2017; Marciano et al. 2014). Similar regulatory processes have been mainly described in the context of mutant genes and the upregulation of compensatory mechanims/genes (ElBrolosy, Kontarakis, et al. 2019; Ma et al. 2019, for a review see Wilkinson 2019). Certain findings, however, suggest similar mechanisms to compensate for gene overexpression (see for example Ji et al. 2013) and it is therefore possible that the additional functional copy of the nompC gene under UAS control does not necessarily result in a significant increase in the net amount of NOMPC protein found in the HC population. Additionally, as will be discussed in more detail below, it is not unlikely that NOMPC relies on a functional interaction with other proteins in the HCs, be it the GR28B(D) receptor, or other, as of yet undiscovered channels/proteins. If a direct interaction with these factors is mandatory in order for NOMPC to fulfill its modulatory function, it is possible that the number of interaction partners for NOMPC might be a limiting factor. If the additional copy of nompC does increase the net amount of protein, but the number of essential interaction partners stays the same, it could mean that the additional NOMPC amount simply is not able to fulfill its function, or maybe even localize correctly.

Though we do not have information on how NOMPC and GR28B(D) might interact, several mechanisms of ion channel regulation and cooperative gating have been described for a range of cells and systems. While it is not clear whether the HC transduction machinery follows any of these established mechanisms, it does provide a possible starting point for further investigation of how temperature sensation in the arista neurons is modulated and regulated. TRP channels themselves have been proposed to be able to form heteromultimeric channels, such as in the case of Nanchung and Inactive (Gong et al. 2004). However, the fact that GR28B(D) is not a member of the TRP family, and that the gustatory receptor has been proven to function as a thermosensitve channel by itself, while NOMPC appears to fulfill a regulatory role, renders such interactions rather unlikely. Another proposed interaction pathway between channels is the so-called cooperative gating (for a review, refer to Choi 2014). Positive cooperativity has been proposed for a number of ion channels, such as voltage gated $\mathrm{Na}^{+}$channels (Naundorf, Wolf, and Volgushev 2006). Different models on the exact way of channel interaction have been proposed, but the difficulty of an assessment in vivo has prevented the confirmation of the proposed mechanisms in biological systems (Choi 2014). The more prominent explanation in which the opening of one ion channels affects the gating of another include: The sensing/binding of ions that are transported across the membrane (mechanism pro- 
posed in Standen and Stanfield 1982). This process has been described as the possible origin of negative feedback regulation for $\mathrm{Ca}^{2+}$ channels (Imredy and Yue 1992; Standen and Stanfield 1982). While the structure of GR28B(D) has to be solved, recent advances in the research of NOMPC have lead to more information on its structure (Jin et al. 2017), and possible binding sites of ions (such has $\mathrm{Ca}^{2+}$ ) have been identified (personal communication, Dr. P. Hehlert). This might provide a possible starting point for how GR28B(D) influences NOMPC. Another proposed mechanism of cooperative ion channel interaction revolves around interaction across the cellular membrane (Ursell et al. 2007). As opening of one channel might lead to changes in membrane stretch or curvature, it might directly influence other ion channels embedded into the same membrane via mechanical activation (Ursell et al. 2007). As NOMPC is a mechanosensitive channel, this might allude to a possible interaction pathway, but as recent research has shown, the ankyrin repeat domain of NOMPC is essential for the force conveyance to the channel (Zhang, Cheng, et al. 2015). And the way in which mechanical stress from another channel could conveyed to another in a cytoskeleton-tethered system remains unclear (Choi 2014).

This are of course only a potential interaction mechanism in the particular case of the HCs. Established models of cooperative gating have been described to possess a number of prerequisites for proper function, such as the co-localization of the involved channel (Choi 2014). As data on the exact cellular localization of both GR28b(D) and NOMPC does not exist yet, it is not possible to evaluate this factor of channel interaction.

As NOMPC has been shown to be mechanotransducer channel, I aimed to investigate whether mechanical stimulation of the arista would result in responses from the arista neurons. Intriguingly, bending of the arista did not elicit any observable fluorescence changes in $\mathrm{Ca}^{2+}$ imaging experiments that correlated with the mechanical stimulation (see 3.4 on page 56). The initial assumption was not that the arista neurons would serve as mechanosensors for several reasons: From a purely information theory based standpoint, it would be problematic to rely on a single set of specialized sensors to encode for different sensory modalities, as this would raise the problem of how to differentiate between the two distinct perceptions. Additionally, as described in 2.4.2 (page 25), I had to fix the joints between the first, second and third antennal segments to deflect and bend the arista in the experimental trials, as otherwise the mechanical manipulation would just result in movement along the antennal joints. This means that in a normal, behavioural context, by the time a mechanical stimulus would begin to bend the arista structure, the joint between the third and second antennal segment would probably be rotated as far as possible, leading to an activation of all stretch receptive neurons in the Johnston's organ. This makes sense in the context of audition, as vibration sensing of the Johnston's organ relies on the stiff coupling of arista and funcile, so that even small deflections of 
the arista can elicit responses in the hearing organ and are not get compensated by a flexible arista. But even if the mechanical stimulation was not behaviourally relevant, the question remains whether the presence of NOMPC would result in an ability to respond to mechanical stimulation that would just not occur outside of experimental conditions. The fact that I could not observe any mechanically evoked responses could be due to a number of factors: While it is of course possible, that the elicited responses were so small in amplitude that they were not observable during $\mathrm{Ca}^{2+}$ imaging.

Taken together, while the exact mechanism by which the temperature signaling is influenced still has to be investigated, it is clear that NOMPC fulfills a distinct modulatory role in the temperature evoked activity of the arista HCs that appears to be independent of its role as a canonical mechanotransducing channel.

\section{3 nompC mutations alter temperature avoidance be- haviour}

The study presented here could determine that NOMPC modulates the physiological responses of arista neurons towards temperature. An immediate follow-up question in this context was, if the alterations to $\mathrm{Ca}^{2+}$ responses observed would also have an effect on the temperature driven behaviour of Drosophila. The analysis of temperature preference and avoidance behaviour has become an important tool to asses the effects of different cellular and molecular mechanisms in the context of temperature sensation (see for example Barbagallo and Garrity 2015; Fowler and Montell 2013; Giraldo et al. 2019; Rosenzweig, Brennan, et al. 2005; Rosenzweig, Kang, and Garrity 2008. As described in 1.3.1 (page 10), Drosophila possess additional temperature sensors outside of the arista, and even a null mutation of nompC did not completely abolish $\mathrm{HC}$ responses. I therefore assessed the temperature preference and avoidance behaviour of different wildtype and mutant strains (See 3.5, starting on page 58).

Heterozygous nompc $\mathrm{C}^{3}$ mutants showed a significantly later onset of hot avoidance behaviour and a higher preferred temperature, while cold avoidance behaviour was not affected. To assess the effect that a loss of the $\mathrm{HC}$ information would have on the behaviour, I also tested flies in which the HC population was ablated using apoptotic factors Hid and Reaper (hid,rpr). Flies with ablated HCs did show later hot avoidance. Interestingly, the effect of the heterozygous nomp $\mathrm{C}^{3}$ appeared to be stronger than the ablation of HCs in this regard, as the difference compared to wildtype controls showed a stronger significance. This might appear counter-intuitive at first, as nompC mutants were shown to still exhibit a $\mathrm{HC}$ response to temperature, although with a greatly reduced amplitude, while an ablation of the cell would eliminate this response. However, the explanation to 
this observation might lie in the integration of conflicting signals from the temperature sensors: As was shown in the original characterization of the arista neurons (Gallio et al. 2011), and again demonstrated in the data of this study, both HC and CC populations exhibit changes in their activity to both temperature increments and decrements, by exhibiting excitatory responses towards one stimulus direction and inhibitory responses to the other directions. This suggests that both cell populations can provide information about both heating and cooling, albeit in a different manner. This could imply that a certain degree of redundancy in the system can compensate for the loss of information of one cell group. In the example of this study, it could mean that although the HC population was ablated and this affected the heat perception of the fly, the CCs could still provide some fundamental information about hot temperatures in the behavioural experiment. The previously discussed physiological experiments of this project suggested that nompC mutants exhibit lower $\mathrm{HC}$ response amplitudes than wildtypes. While the responses of CCs are also altered to some degree, the effect was stronger in the HCs. Information about the intensity of temperature change appears to be represented by the response amplitude. However, both HCs and CCs likely provide information about the temperature change, but with response amplitudes that suggest different temperature changes. This might in the end affect the integration of the sensory data, and ultimately the temperature driven behaviour, stronger than a simple loss of information from nonfunctional HCs. Conciliation of conflicting sensory information is a complex topic in all sensor driven systems, from biological systems (see for example Cloke, Jacklin, and Winters 2015; Fetsch, DeAngelis, and Angelaki 2013) all the way to technical applications of sensor fusion theory (Xiao and Qin 2018). Even in the wild, Drosophila has to deal with conflicting sensory information, as has for example been studied in the context of olfactory information during different behavioural situations, such as feeding (Lewis et al. 2015). In cases like this, complex higher order processing can be used to evaluate the different sensory inputs in order to reach a reliable state for decision making. If the conflicting sensory information does however originate from a sensory system that is not working "as intended", as would be the case in the nompC mutant flies examined in this study, it is improbable that compensatory or processing mechanisms can properly evaluate the obtained information. This might provide a possible explanation for the observed strong effect of nompC mutations in regards to temperature avoidance behaviour, when compared to wildtypes and animals with ablated HCs.

The behavioural effects analyzed in this study further underline the importance of the arista neurons as a first response system to ambient temperature changes, as even abolishment of only one of the two cell groups, and mutations that resulted in response amplitude redcution, but not elimination, lead to significant effects in the avoidance behaviour of adult Drosophila. I could furhtermore show that NOMPCs role in modulating 
temperature evoked neuronal responses is relevant in a behavioural context, and even a heterozygous knockout of the nompC gene is not easily compensated for by other thermosensitive systems in the fly.

To conclude, this study illustrates that the neurons found in Drosophilas arista are relative temperature sensors, whose responses appear to be modulated by the TRP channel NOMPC. I showed that both HCs and CCs can theoretically provide information about an increase or decrease in temperature, wherein higher changes in temperature lead to stronger $\mathrm{Ca}^{2+}$ response amplitudes in the cells. Expression of nompC found in the $\mathrm{HC}$ population and NOMPC appears to play an active role in the amplitude modulation of temperature evoked $\mathrm{Ca}^{2+}$ responses of the arista neurons. 


\section{Chapter 5}

\section{References}

\section{References}

Abram, Paul K, Guy Boivin, Joffrey Moiroux, and Jacques Brodeur (2017). "Behavioural effects of temperature on ectothermic animals: unifying thermal physiology and behavioural plasticity". In: Biological Reviews 92.4, pages 1859-1876.

Ahmad, Syed Tariq, Michael Natochin, Nikolai O Artemyev, and Joseph E O'Tousa (2007). "The Drosophila rhodopsin cytoplasmic tail domain is required for maintenance of rhabdomere structure". In: The FASEB Journal 21.2, pages 449-455.

Akerboom, Jasper, Jonathan D Vélez Rivera, Marıa M Rodrıguez Guilbe, Elisa C Alfaro Malavé, Hector H Hernandez, Lin Tian, S Andrew Hires, Jonathan S Marvin, Loren L Looger, and Eric R Schreiter (2009). "Crystal structures of the GCaMP calcium sensor reveal the mechanism of fluorescence signal change and aid rational design". In: Journal of biological chemistry 284.10, pages 6455-6464.

Akitake, Bradley, Qiuting Ren, Nina Boiko, Jinfei Ni, Takaaki Sokabe, James D Stockand, Benjamin A Eaton, and Craig Montell (2015). "Coordination and fine motor control depend on Drosophila TRP $\gamma$ ". In: Nature communications 6, page 7288.

Andersson, David A, Henry WN Chase, and Stuart Bevan (2004). "TRPM8 activation by menthol, icilin, and cold is differentially modulated by intracellular $\mathrm{pH}^{\prime \prime}$. In: Journal of Neuroscience 24.23, pages 5364-5369.

Andersson, David A, Clive Gentry, Sian Moss, and Stuart Bevan (2008). "Transient receptor potential A1 is a sensory receptor for multiple products of oxidative stress". In: Journal of Neuroscience 28.10, pages 2485-2494.

Aneiros, Eduardo, Lishuang Cao, Marianthi Papakosta, Edward B Stevens, Stephen Phillips, and Christian Grimm (2011). "The biophysical and molecular basis of TRPV1 proton gating". In: The EMBO journal 30.6, pages 994-1002. 
Al-Anzi, Bader, W Daniel Tracey Jr, and Seymour Benzer (2006). "Response of Drosophila to wasabi is mediated by painless, the fly homolog of mammalian TRPA1/ANKTM1". In: Current Biology 16.10, pages 1034-1040.

Attia, Moneim (1984). "Thermal pleasantness and temperature regulation in man". In: Neuroscience \& Biobehavioral Reviews 8.3, pages 335-342.

Barbagallo, Belinda and Paul A Garrity (2015). "Temperature sensation in Drosophila". In: Current Opinion in Neurobiology 34, pages 8-13.

Bautista, Diana M, Jan Siemens, Joshua M Glazer, Pamela R Tsuruda, Allan I Basbaum, Cheryl L Stucky, Sven-Eric Jordt, and David Julius (2007). "The menthol receptor TRPM8 is the principal detector of environmental cold". In: Nature 448.7150, page 204.

Benjamini, Yoav and Yosef Hochberg (1995). "Controlling the false discovery rate: a practical and powerful approach to multiple testing". In: Journal of the Royal statistical society: series B (Methodological) 57.1, pages 289-300.

Birnbaumer, Lutz (2009). "The TRPC class of ion channels: a critical review of their roles in slow, sustained increases in intracellular Ca2+ concentrations". In: Annual review of pharmacology and toxicology 49, pages 395-426.

Boekhoff-Falk, Grace (2005). "Hearing in Drosophila: development of Johnston's organ and emerging parallels to vertebrate ear development". In: Developmental dynamics: an official publication of the American Association of Anatomists 232.3, pages 550558.

Brand, Andrea H and Norbert Perrimon (1993). "Targeted gene expression as a means of altering cell fates and generating dominant phenotypes". In: development 118.2, pages 401-415.

Briffa, Mark, Danielle Bridger, and Peter A Biro (2013). "How does temperature affect behaviour? Multilevel analysis of plasticity, personality and predictability in hermit crabs". In: Animal Behaviour 86.1, pages 47-54.

Brohawn, Stephen G, Zhenwei Su, and Roderick MacKinnon (2014). "Mechanosensitivity is mediated directly by the lipid membrane in TRAAK and TREK1 $\mathrm{K}+$ channels". In: Proceedings of the National Academy of Sciences 111.9, pages 3614-3619.

El-Brolosy, Mohamed A, Zacharias Kontarakis, Andrea Rossi, Carsten Kuenne, Stefan Guenther, Nana Fukuda, Khrievono Kikhi, Giulia LM Boezio, Carter M Takacs, Shih-Lei Lai, et al. (2019). "Genetic compensation triggered by mutant mRNA degradation". In: Nature 568.7751, page 193.

El-Brolosy, Mohamed A and Didier YR Stainier (2017). "Genetic compensation: a phenomenon in search of mechanisms". In: PLoS genetics 13.7, e1006780.

Budelli, Gonzalo, Lina Ni, Cristina Berciu, Lena van Giesen, Zachary A Knecht, Elaine C Chang, Benjamin Kaminski, Ana F Silbering, Aravi Samuel, Mason Klein, et al. (2019). 
"Ionotropic Receptors Specify the Morphogenesis of Phasic Sensors Controlling Rapid Thermal Preference in Drosophila". In: Neuron.

Cai, Xinjiang (2006). "Unicellular Ca2+ signaling 'toolkit' at the origin of metazoa". In: Molecular biology and evolution 25.7, pages 1357-1361.

Cai, Xinjiang and David E Clapham (2011). "Ancestral Ca2+ signaling machinery in early animal and fungal evolution". In: Molecular biology and evolution 29.1, pages 91-100.

Caldwell, Jason C and Daniel F Eberl (2002). "Towards a molecular understanding of Drosophila hearing". In: Journal of neurobiology 53.2, pages 172-189.

Carlson, Neil R (1994). Physiology of behavior. Allyn \& Bacon.

Caterina, Michael J, Mark A Schumacher, Makoto Tominaga, Tobias A Rosen, Jon D Levine, and David Julius (1997). "The capsaicin receptor: a heat-activated ion channel in the pain pathway". In: Nature 389.6653, page 816.

Chemin, Jean, Amanda Jane Patel, Fabrice Duprat, Inger Lauritzen, Michel Lazdunski, and Eric Honoré (2005). "A phospholipid sensor controls mechanogating of the K+ channel TREK-1". In: The EMBO journal 24.1, pages 44-53.

Chen, Tsai-Wen, Trevor J Wardill, Yi Sun, Stefan R Pulver, Sabine L Renninger, Amy Baohan, Eric R Schreiter, Rex A Kerr, Michael B Orger, Vivek Jayaraman, et al. (2013). "Ultrasensitive fluorescent proteins for imaging neuronal activity". In: Nature 499.7458, page 295.

Cheng, Li E, Wei Song, Loren L Looger, Lily Yeh Jan, and Yuh Nung Jan (2010). "The role of the TRP channel NompC in Drosophila larval and adult locomotion". In: Neuron 67.3, pages 373-380.

Choi, Kee-Hyun (2014). "Cooperative gating between ion channels." In: General physiology and biophysics 33.1, pages 1-12.

Chowdhury, Sandipan, Brian W Jarecki, and Baron Chanda (2014). "A molecular framework for temperature-dependent gating of ion channels". In: Cell 158.5, pages 11481158.

Clapham, David E, Craig Montell, Guenter Schultz, and David Julius (2003). "International Union of Pharmacology. XLIII. Compendium of voltage-gated ion channels: transient receptor potential channels". In: Pharmacological Reviews 55.4, pages 591-596.

Clapham, David E, Loren W Runnels, and Carsten Strübing (2001). "The TRP ion channel family". In: Nature Reviews Neuroscience 2.6, page 387.

Cloke, Jacob M, Derek L Jacklin, and Boyer D Winters (2015). "The neural bases of crossmodal object recognition in non-human primates and rodents: a review". In: Behavioural brain research 285, pages 118-130.

Cordero-Morales, Julio F, Elena O Gracheva, and David Julius (2011). "Cytoplasmic ankyrin repeats of transient receptor potential A1 (TRPA1) dictate sensitivity to thermal and chemical stimuli". In: Proceedings of the National Academy of Sciences 108.46, E1184E1191. 
Denis, Valérie and Martha S Cyert (2002). "Internal Ca2+ release in yeast is triggered by hypertonic shock and mediated by a TRP channel homologue". In: J Cell Biol 156.1, pages 29-34.

Dhaka, Ajay, Veena Viswanath, and Ardem Patapoutian (2006). "Trp ion channels and temperature sensation". In: Annu. Rev. Neurosci. 29, pages 135-161.

Dillon, Michael E, George Wang, Paul A Garrity, and Raymond B Huey (2009). "Thermal preference in Drosophila". In: Journal of thermal biology 34.3, pages 109-119.

Dong, Xian-Ping, Xiang Wang, and Haoxing Xu (2010). "TRP channels of intracellular membranes". In: Journal of neurochemistry 113.2, pages 313-328.

Effertz, Thomas, Robert Wiek, and Martin C Göpfert (2011). "NompC TRP channel is essential for Drosophila sound receptor function". In: Current Biology 21.7, pages 592597.

Enjin, Anders, Emanuela E Zaharieva, Dominic D Frank, Suzan Mansourian, Greg SB Suh, Marco Gallio, and Marcus C Stensmyr (2016). "Humidity sensing in Drosophila". In: Current Biology 26.10, pages 1352-1358.

Enyedi, Péter and Gábor Czirják (2010). "Molecular background of leak K+ currents: twopore domain potassium channels". In: Physiological reviews 90.2, pages 559-605.

Fetsch, Christopher R, Gregory C DeAngelis, and Dora E Angelaki (2013). "Bridging the gap between theories of sensory cue integration and the physiology of multisensory neurons". In: Nature Reviews Neuroscience 14.6, page 429.

Florence, TJ and Michael B Reiser (2015). "Neuroscience: hot on the trail of temperature processing". In: Nature 519.7543, page 296.

Flouris, Andreas D (2011). "Functional architecture of behavioural thermoregulation". In: European journal of applied physiology 111.1, pages 1-8.

Foelix, RF, RF Stocker, and RA Steinbrecht (1989). "Fine structure of a sensory organ in the arista of Drosophila melanogaster and some other dipterans". In: Cell and tissue research 258.2, pages 277-287.

Fowler, Melissa A and Craig Montell (2013). "Drosophila TRP channels and animal behavior". In: Life sciences 92.8-9, pages 394-403.

Frank, Dominic D, Genevieve C Jouandet, Patrick J Kearney, Lindsey J Macpherson, and Marco Gallio (2015). "Temperature representation in the Drosophila brain". In: Nature 519.7543 , page 358 .

Frank, Steven A (2013). "Input-output relations in biological systems: measurement, information and the Hill equation". In: Biology direct 8.1, page 31.

Frank, Vered, Germán E Piñas, Harel Cohen, John S Parkinson, and Ady Vaknin (2016). "Networked chemoreceptors benefit bacterial chemotaxis performance". In: MBio 7.6, e01824-16. 
Gallio, Marco, Tyler A Ofstad, Lindsey J Macpherson, Jing W Wang, and Charles S Zuker (2011). "The coding of temperature in the Drosophila brain". In: Cell 144.4, pages 614624.

Gao, Zhiqian, Douglas M Ruden, and Xiangyi Lu (2003). "PKD2 cation channel is required for directional sperm movement and male fertility". In: Current Biology 13.24, pages 2175-2178.

Garrity, Paul A, Miriam B Goodman, Aravinthan D Samuel, and Piali Sengupta (2010). "Running hot and cold: behavioral strategies, neural circuits, and the molecular machinery for thermotaxis in C. elegans and Drosophila". In: Genes \& development 24.21, pages 2365-2382.

Gaudet, Rachelle (2008). "A primer on ankyrin repeat function in TRP channels and beyond". In: Molecular BioSystems 4.5, pages 372-379.

Georgiev, Plamen, Hanneke Okkenhaug, Anna Drews, David Wright, Sachar Lambert, Melanie Flick, Valentina Carta, Cecile Martel, Johannes Oberwinkler, and Padinjat Raghu (2010). "TRPM channels mediate zinc homeostasis and cellular growth during Drosophila larval development". In: Cell metabolism 12.4, pages 386-397.

Giraldo Sanchez, Diego Alejandro (2018). "Linking Senses: the Genetics of Drosophila Larval Chordotonal Organs". PhD thesis. Georg-August-Universität Göttingen.

Giraldo, Diego, Andrea Adden, Ilyas Kuhlemann, Heribert Gras, and Bart RH Geurten (2019). "Correcting locomotion dependent observation biases in thermal preference of Drosophila". In: Scientific reports 9.1, page 3974.

Gong, Zhefeng, Wonseok Son, Yun Doo Chung, Janghwan Kim, Dong Wook Shin, Colleen A McClung, Yong Lee, Hye Won Lee, Deok-Jin Chang, Bong-Kiun Kaang, et al. (2004). "Two interdependent TRPV channel subunits, inactive and Nanchung, mediate hearing in Drosophila". In: Journal of Neuroscience 24.41, pages 9059-9066.

Göpfert, Martin C, Jörg T Albert, B Nadrowski, and A Kamikouchi (2006). "Specification of auditory sensitivity by Drosophila TRP channels". In: Nature neuroscience 9.8, page 999.

Göpfert, Martin C and Daniel Robert (2001). "Biomechanics: Turning the key on Drosophila audition". In: Nature 411.6840, page 908.

- (2002). "The mechanical basis of Drosophila audition". In: Journal of Experimental Biology 205.9, pages 1199-1208.

- (2003). "Motion generation by Drosophila mechanosensory neurons". In: Proceedings of the National Academy of Sciences 100.9, pages 5514-5519.

Grether, Megan E, John M Abrams, Julie Agapite, Kristin White, and Hermann Steller (1995). "The head involution defective gene of Drosophila melanogaster functions in programmed cell death." In: Genes \& development 9.14, pages 1694-1708. 
Groppe, David M, Thomas P Urbach, and Marta Kutas (2011). "Mass univariate analysis of event-related brain potentials/fields I: A critical tutorial review". In: Psychophysiology 48.12, pages 1711-1725.

Guntur, Ananya R, Pengyu Gu, Kendra Takle, Jingyi Chen, Yang Xiang, and Chung-Hui Yang (2015). "Drosophila TRPA1 isoforms detect UV light via photochemical production of H2O2". In: Proceedings of the National Academy of Sciences 112.42, E5753-E5761.

Hamada, Fumika N, Mark Rosenzweig, Kyeongjin Kang, Stefan R Pulver, Alfredo Ghezzi, Timothy J Jegla, and Paul A Garrity (2008). "An internal thermal sensor controlling temperature preference in Drosophila". In: Nature 454.7201, page 217.

Heinrich, Bernd (2013). The hot-blooded insects: strategies and mechanisms of thermoregulation. Springer Science \& Business Media.

Hepker, Jennifer, Qun-Tian Wang, Cynthia K Motzny, Robert Holmgren, and Teresa Vales Orenic (1997). "Drosophila cubitus interruptus forms a negative feedback loop with patched and regulates expression of Hedgehog target genes". In: Development 124.2, pages 549-558.

Hofmann, Thomas, Vladimir Chubanov, Xiaodi Chen, Anna S Dietz, Thomas Gudermann, and Craig Montell (2010). "Drosophila TRPM channel is essential for the control of extracellular magnesium levels". In: PloS one 5.5, e10519.

Huey, Raymond B, Paul E Hertz, and B Sinervo (2003). "Behavioral drive versus behavioral inertia in evolution: a null model approach". In: The American Naturalist 161.3, pages 357-366.

Hwang, Richard Y, Nancy A Stearns, and W Daniel Tracey (2012). "The ankyrin repeat domain of the TRPA protein painless is important for thermal nociception but not mechanical nociception". In: PloS one 7.1, e30090.

Imredy, John P and David T Yue (1992). "Submicroscopic Ca2+ diffusion mediates inhibitory coupling between individual Ca2+ channels". In: Neuron 9.2, pages 197-207. Jarman, Andrew P, Yan Sun, Lily Y Jan, and Yuh Nung Jan (1995). "Role of the proneural gene, atonal, in formation of Drosophila chordotonal organs and photoreceptors". In: Development 121.7, pages 2019-2030.

Ji, Ni, Teije C Middelkoop, Remco A Mentink, Marco C Betist, Satto Tonegawa, Dylan Mooijman, Hendrik C Korswagen, and Alexander van Oudenaarden (2013). "Feedback control of gene expression variability in the Caenorhabditis elegans Wnt pathway". In: Cell 155.4, pages 869-880.

Jin, Peng, David Bulkley, Yanmeng Guo, Wei Zhang, Zhenhao Guo, Walter Huynh, Shenping Wu, Shan Meltzer, Tong Cheng, Lily Yeh Jan, et al. (2017). "Electron cryomicroscopy structure of the mechanotransduction channel NOMPC". In: Nature 547.7661, page 118 .

Junger, Wolfgang G (2011). "Immune cell regulation by autocrine purinergic signalling". In: Nature Reviews Immunology 11.3, page 201. 
Kadowaki, Tatsuhiko (2015). "Evolutionary dynamics of metazoan TRP channels". In: Pflügers Archiv-European Journal of Physiology 467.10, pages 2043-2053.

Kandel, Eric R, James H Schwartz, Thomas M Jessell, Department of Biochemistry, Molecular Biophysics Thomas Jessell, Steven Siegelbaum, and AJ Hudspeth (2000). Principles of neural science. McGraw-hill New York.

Kang, Kyeongjin, Stefan R Pulver, Vincent C Panzano, Elaine C Chang, Leslie C Griffith, Douglas L Theobald, and Paul A Garrity (2010). "Analysis of Drosophila TRPA1 reveals an ancient origin for human chemical nociception". In: Nature 464.7288, page 597.

Kang, Yoona, Lawrence E Williams, Margaret S Clark, Jeremy R Gray, and John A Bargh (2010). "Physical temperature effects on trust behavior: the role of insula". In: Social cognitive and affective neuroscience 6.4, pages 507-515.

Kernan, Maurice, David Cowan, and Charles Zuker (1994). "Genetic dissection of mechanosensory transduction: mechanoreception-defective mutations of Drosophila". In: Neuron 12.6, pages 1195-1206.

Kim, Janghwan, Yun Doo Chung, Dae-young Park, SooKyung Choi, Dong Wook Shin, Heun Soh, Hye Won Lee, Wonseok Son, Jeongbin Yim, Chul-Seung Park, et al. (2003). "A TRPV family ion channel required for hearing in Drosophila". In: Nature 424.6944, page 81.

Kim, Sang Hoon, Youngseok Lee, Bradley Akitake, Owen M Woodward, William B Guggino, and Craig Montell (2010). "Drosophila TRPA1 channel mediates chemical avoidance in gustatory receptor neurons". In: Proceedings of the National Academy of Sciences 107.18, pages 8440-8445.

Kim, Susy M and Jing W Wang (2016). "Hygrosensation: feeling wet and cold". In: Current Biology 26.10, R408-R410.

Klein, Mason, Bruno Afonso, Ashley J Vonner, Luis Hernandez-Nunez, Matthew Berck, Christopher J Tabone, Elizabeth A Kane, Vincent A Pieribone, Michael N Nitabach, Albert Cardona, et al. (2015). "Sensory determinants of behavioral dynamics in Drosophila thermotaxis". In: Proceedings of the National Academy of Sciences 112.2, E220-E229.

Klepsatel, Peter, Martina Gáliková, Yanjun Xu, and Ronald P Kühnlein (2016). "Thermal stress depletes energy reserves in Drosophila". In: Scientific reports 6, page 33667.

Knecht, Zachary A, Ana F Silbering, Lina Ni, Mason Klein, Gonzalo Budelli, Rati Bell, Liliane Abuin, Anggie J Ferrer, Aravinthan DT Samuel, Richard Benton, et al. (2016). "Distinct combinations of variant ionotropic glutamate receptors mediate thermosensation and hygrosensation in Drosophila". In: Elife 5, e17879.

Köttgen, Michael, Alexis Hofherr, Weizhe Li, Kristy Chu, Stacey Cook, Craig Montell, and Terry Watnick (2011). "Drosophila sperm swim backwards in the female reproductive tract and are activated via TRPP2 ion channels". In: PLoS One 6.5, e20031. 
Kumar, Justin P and Donald F Ready (1995). "Rhodopsin plays an essential structural role in Drosophila photoreceptor development". In: Development 121.12, pages 4359-4370.

Kwon, Young, Wei L Shen, Hye-Seok Shim, and Craig Montell (2010). "Fine thermotactic discrimination between the optimal and slightly cooler temperatures via a TRPV channel in chordotonal neurons". In: Journal of Neuroscience 30.31, pages 10465-10471.

Kwon, Young, Hye-Seok Shim, Xiaoyue Wang, and Craig Montell (2008). "Control of thermotactic behavior via coupling of a TRP channel to a phospholipase $\mathrm{C}$ signaling cascade". In: Nature neuroscience 11.8, page 871.

Ladrón-de-Guevara, E, GE Rangel-Yescas, DA Fernández-Velasco, A Torres-Larios, T Rosenbaum, and LD Islas (2019). "The contribution of the ankyrin repeat domain of TRPV1 as a thermal module". In: bioRxiv, page 641803.

Lai, Sen-Lin and Tzumin Lee (2006). "Genetic mosaic with dual binary transcriptional systems in Drosophila". In: Nature neuroscience 9.5, page 703.

Lam, Tania and Keir G Pearson (2002). "The role of proprioceptive feedback in the regulation and adaptation of locomotor activity". In: Sensorimotor Control of Movement and Posture. Springer, pages 343-355.

Latorre, Ramon, Sebastian Brauchi, Gerardo Orta, Cristián Zaelzer, and Guillermo Vargas (2007). "ThermoTRP channels as modular proteins with allosteric gating". In: Cell calcium 42.4-5, pages 427-438.

Latorre, Ramon, Guillermo Vargas, Gerardo Orta, and Sebastian Brauchi (2006). "Voltage and Temperature Gating of ThermoTRP Channels". In: TRP ion channel function in sensory transduction and cellular signaling cascades, page 287.

Lee, Jeongmi, Sungjin Moon, Yoonseok Cha, and Yun Doo Chung (2010). "Drosophila TRPN (= NOMPC) channel localizes to the distal end of mechanosensory cilia". In: PLoS One 5.6, e11012.

Lee, Youngseok, Yong Lee, Jaejung Lee, Sunhoe Bang, Seogang Hyun, Jongkyun Kang, Sung-Tae Hong, Eunkyung Bae, Bong-Kiun Kaang, and Jaeseob Kim (2005). "Pyrexia is a new thermal transient receptor potential channel endowing tolerance to high temperatures in Drosophila melanogaster". In: Nature genetics 37.3, page 305.

Lewis, Laurence PC, KP Siju, Yoshinori Aso, Anja B Friedrich, Alexander JB Bulteel, Gerald M Rubin, and Ilona C Grunwald Kadow (2015). "A higher brain circuit for immediate integration of conflicting sensory information in Drosophila". In: Current biology 25.17, pages 2203-2214.

Li, Wei, Zhaoyang Feng, Paul W Sternberg, and XZ Shawn Xu (2006). "A C. elegans stretch receptor neuron revealed by a mechanosensitive TRP channel homologue". In: Nature 440.7084, page 684.

Liang, Xin, Johnson Madrid, Henri S Saleh, and Jonathon Howard (2011). "NOMPC, a member of the TRP channel family, localizes to the tubular body and distal cilium 
of Drosophila campaniform and chordotonal receptor cells". In: Cytoskeleton 68.1, pages 1-7.

Lishko, Polina V, Erik Procko, Xiangshu Jin, Christopher B Phelps, and Rachelle Gaudet (2007). "The ankyrin repeats of TRPV1 bind multiple ligands and modulate channel sensitivity". In: Neuron 54.6, pages 905-918.

Liu, Lei, Yuhong Li, Runping Wang, Chong Yin, Qian Dong, Huey Hing, Changsoo Kim, and Michael J Welsh (2007). "Drosophila hygrosensation requires the TRP channels water witch and nanchung". In: Nature 450.7167, page 294.

Liu, Lei, Olena Yermolaieva, Wayne A Johnson, Francois M Abboud, and Michael J Welsh (2003). "Identification and function of thermosensory neurons in Drosophila larvae". In: Nature neuroscience 6.3, page 267.

Liu, Wendy W, Ofer Mazor, and Rachel I Wilson (2015). "Thermosensory processing in the Drosophila brain". In: Nature 519.7543, page 353.

Luo, Junjie, Wei L Shen, and Craig Montell (2017). "TRPA1 mediates sensation of the rate of temperature change in Drosophila larvae". In: Nature neuroscience 20.1, page 34. Ma, Zhipeng, Peipei Zhu, Hui Shi, Liwei Guo, Qinghe Zhang, Yanan Chen, Shuming Chen, Zhe Zhang, Jinrong Peng, and Jun Chen (2019). "PTC-bearing mRNA elicits a genetic compensation response via Upf3a and COMPASS components". In: Nature 568.7751, page 259.

Maingret, François, Inger Lauritzen, Amanda J Patel, Catherine Heurteaux, Roberto Reyes, Florian Lesage, Michel Lazdunski, and Eric Honoré (2000). "TREK-1 is a heat-activated background K+ channel". In: The EMBO journal 19.11, pages 2483-2491.

Maingret, François, Amanda J Patel, Florian Lesage, Michel Lazdunski, and Eric Honoré (1999). "Mechano-or acid stimulation, two interactive modes of activation of the TREK-1 potassium channel". In: Journal of Biological Chemistry 274.38, pages 2669126696.

- (2000). "Lysophospholipids open the two-pore domain mechano-gated K+ channels TREK-1 and TRAAK". In: Journal of Biological Chemistry 275.14, pages 10128-10133.

Marciano, David C, Rhonald C Lua, Panagiotis Katsonis, Shivas R Amin, Christophe Herman, and Olivier Lichtarge (2014). "Negative feedback in genetic circuits confers evolutionary resilience and capacitance". In: Cell reports 7.6, pages 1789-1795.

Marija, Jakšić Ana, Karner Julia, Nolte Viola, Hsu Sheng-Kai, Barghi Neda, Mallard François, Otte Kathrin Anna, Svečnjak Lidija, Senti Kirsten-André, and Schlötterer Christian (2019). "Neuronal function and dopamine signaling evolve at high temperature in Drosophila". In: bioRxiv, page 585422.

McKemy, David D, Werner M Neuhausser, and David Julius (2002). "Identification of a cold receptor reveals a general role for TRP channels in thermosensation". In: Nature 416.6876, page 52 . 
Menon, Indu, Thomas Huber, Sumana Sanyal, Sourabh Banerjee, Patrick Barré, Sam Canis, J David Warren, John Hwa, Thomas P Sakmar, and Anant K Menon (2011). "Opsin is a phospholipid flippase". In: Current Biology 21.2, pages 149-153.

Mishra, Aditi, Autoosa Salari, Benton R Berigan, Kayla C Miguel, Marzie Amirshenava, Abbey Robinson, Benjamin C Zars, Jenna L Lin, Lorin S Milescu, Mirela Milescu, et al. (2018). "The Drosophila Gr28bD product is a non-specific cation channel that can be used as a novel thermogenetic tool". In: Scientific reports 8.1, page 901.

Montell, Craig (2005). "The TRP superfamily of cation channels". In: Science Signaling 2005.272, re3-re3.

- (2012). "Drosophila visual transduction". In: Trends in neurosciences 35.6, pages 356363.

- (2013). "Gustatory receptors: not just for good taste". In: Current Biology 23.20, R929R932.

Montell, Craig, Lutz Birnbaumer, Veit Flockerzi, René J Bindels, Elspeth A Bruford, Michael J Caterina, David E Clapham, Christian Harteneck, Stefan Heller, David Julius, et al. (2002). "A unified nomenclature for the superfamily of TRP cation channels". In: Molecular cell 9.2, pages 229-231.

Moyes, Christopher D and Patricia M Schulte (2008). Tierphysiologie. Pearson Deutschland $\mathrm{GmbH}$.

Mullur, Rashmi, Yan-Yun Liu, and Gregory A Brent (2014). "Thyroid hormone regulation of metabolism". In: Physiological reviews 94.2, pages 355-382.

Nakai, Junichi, Masamichi Ohkura, and Keiji Imoto (2001). "A high signal-to-noise Ca 2+ probe composed of a single green fluorescent protein". In: Nature biotechnology 19.2, page 137.

Naundorf, Björn, Fred Wolf, and Maxim Volgushev (2006). "Unique features of action potential initiation in cortical neurons". In: Nature 440.7087, page 1060.

Nealen, Michele L, Michael S Gold, Paul D Thut, and Michael J Caterina (2003). "TRPM8 mRNA is expressed in a subset of cold-responsive trigeminal neurons from rat". In: Journal of neurophysiology 90.1, pages 515-520.

Neely, G Gregory, Alex C Keene, Peter Duchek, Elaine C Chang, Qiao-Ping Wang, Yagiz Alp Aksoy, Mark Rosenzweig, Michael Costigan, Clifford J Woolf, Paul A Garrity, et al. (2011). "TrpA1 regulates thermal nociception in Drosophila". In: PloS one 6.8, e24343.

$\mathrm{Ni}$, Lina, Peter Bronk, Elaine C Chang, April M Lowell, Juliette O Flam, Vincent C Panzano, Douglas L Theobald, Leslie C Griffith, and Paul A Garrity (2013). "A gustatory receptor paralogue controls rapid warmth avoidance in Drosophila". In: Nature 500.7464, page 580.

Ni, Lina, Mason Klein, Kathryn V Svec, Gonzalo Budelli, Elaine C Chang, Anggie J Ferrer, Richard Benton, Aravinthan DT Samuel, and Paul A Garrity (2016). "The ionotropic receptors IR21a and IR25a mediate cool sensing in Drosophila". In: Elife 5, e13254. 
Niwa, Nao, Yasushi Hiromi, and Masataka Okabe (2004). "A conserved developmental program for sensory organ formation in Drosophila melanogaster". In: Nature genetics 36.3, page 293.

Noël, Jacques, Katharina Zimmermann, Jérome Busserolles, Emanuel Deval, Abdelkrim Alloui, Sylvie Diochot, Nicolas Guy, Marc Borsotto, Peter Reeh, Alain Eschalier, et al. (2009). "The mechano-activated K+ channels TRAAK and TREK-1 control both warm and cold perception". In: The EMBO journal 28.9, pages 1308-1318.

Palmer, Chris P, Xin-Liang Zhou, Junyu Lin, Stephen H Loukin, Ching Kung, and Yoshiro Saimi (2001). "A TRP homolog in Saccharomyces cerevisiae forms an intracellular Ca2+-permeable channel in the yeast vacuolar membrane". In: Proceedings of the National Academy of Sciences 98.14, pages 7801-7805.

Pan, Zan, Hua Yang, and Peter S Reinach (2011). "Transient receptor potential (TRP) gene superfamily encoding cation channels". In: Human genomics 5.2, page 108.

Parkinson, John S, Gerald L Hazelbauer, and Joseph J Falke (2015). "Signaling and sensory adaptation in Escherichia coli chemoreceptors: 2015 update". In: Trends in microbiology 23.5 , pages $257-266$.

Partridge, Derek, Patricia D Lopez, and Victor S Johnston (1984). "Computer programs as theories in biology". In: Journal of theoretical biology 108.4, pages 539-564.

Paulsen, Candice E, Jean-Paul Armache, Yuan Gao, Yifan Cheng, and David Julius (2015). "Structure of the TRPA1 ion channel suggests regulatory mechanisms". In: Nature 520.7548, page 511.

Pearson, Keir G (1995). "Proprioceptive regulation of locomotion". In: Current opinion in neurobiology 5.6, pages 786-791.

Peier, Andrea M, Aziz Moqrich, Anne C Hergarden, Alison J Reeve, David A Andersson, Gina M Story, Taryn J Earley, Ilaria Dragoni, Peter McIntyre, Stuart Bevan, et al. (2002). "A TRP channel that senses cold stimuli and menthol". In: Cell 108.5, pages 705-715.

Peng, Guangda, Xiao Shi, and Tatsuhiko Kadowaki (2015). "Evolution of TRP channels inferred by their classification in diverse animal species". In: Molecular phylogenetics and evolution 84, pages 145-157.

Pereira, Vanessa, Jérôme Busserolles, Marine Christin, Maıly Devilliers, Laura Poupon, Wassim Legha, Abdelkrim Alloui, Youssef Aissouni, Emmanuel Bourinet, Florian Lesage, et al. (2014). "Role of the TREK2 potassium channel in cold and warm thermosensation and in pain perception". In: PAIN ${ }^{\circledR} 155.12$, pages $2534-2544$.

Plant, Leigh Daniel (2012). "A role for K2P channels in the operation of somatosensory nociceptors". In: Frontiers in molecular neuroscience 5, page 21.

Ramsey, I Scott, Markus Delling, and David E Clapham (2006). "An introduction to TRP channels". In: Annu. Rev. Physiol. 68, pages 619-647. 
Romanovsky, Andrej A (2007). "Thermoregulation: some concepts have changed. Functional architecture of the thermoregulatory system". In: American journal of Physiology-Regulatory, integrative and comparative Physiology 292.1, R37-R46.

Rosenzweig, Mark, Karen M Brennan, Timothy D Tayler, Paul O Phelps, Ardem Patapoutian, and Paul A Garrity (2005). "The Drosophila ortholog of vertebrate TRPA1 regulates thermotaxis". In: Genes \& development 19.4, pages 419-424.

Rosenzweig, Mark, KyeongJin Kang, and Paul A Garrity (2008). "Distinct TRP channels are required for warm and cool avoidance in Drosophila melanogaster". In: Proceedings of the National Academy of Sciences 105.38, pages 14668-14673.

Salazar, Héctor, Itzel Llorente, Andrés Jara-Oseguera, Refugio Garcla-Villegas, Mika Munari, Sharona E Gordon, León D Islas, and Tamara Rosenbaum (2008). "A single Nterminal cysteine in TRPV1 determines activation by pungent compounds from onion and garlic". In: Nature neuroscience 11.3, page 255.

Sayeed, Omer and Seymour Benzer (1996). "Behavioral genetics of thermosensation and hygrosensation in Drosophila". In: Proceedings of the National Academy of Sciences 93.12, pages 6079-6084.

Schneider, Eve R, Evan O Anderson, Elena O Gracheva, and Sviatoslav N Bagriantsev (2014). "Temperature sensitivity of two-pore (K2P) potassium channels". In: Current topics in membranes. Volume 74. Elsevier, pages 113-133.

Senthilan, Pingkalai R, David Piepenbrock, Guvanch Ovezmyradov, Björn Nadrowski, Susanne Bechstedt, Stephanie Pauls, Margret Winkler, Wiebke Möbius, Jonathon Howard, and Martin C Göpfert (2012). "Drosophila auditory organ genes and genetic hearing defects". In: Cell 150.5, pages 1042-1054.

Sheen, Jen (1994). "Feedback control of gene expression". In: Photosynthesis Research 39.3, pages $427-438$.

Shen, Wei L, Young Kwon, Abidemi A Adegbola, Junjie Luo, Andrew Chess, and Craig Montell (2011). "Function of rhodopsin in temperature discrimination in Drosophila". In: Science 331.6022, pages 1333-1336.

Shettleworth, Sara J (2001). "Animal cognition and animal behaviour". In: Animal behaviour 61.2, pages 277-286.

Shin, Jung-Bum, Dany Adams, Martin Paukert, Maria Siba, Samuel Sidi, Michael Levin, Peter G Gillespie, and Stefan Gründer (2005). "Xenopus TRPN1 (NOMPC) localizes to microtubule-based cilia in epithelial cells, including inner-ear hair cells". In: Proceedings of the National Academy of Sciences 102.35, pages 12572-12577.

Sidi, Samuel, Rainer W Friedrich, and Teresa Nicolson (2003). "NompC TRP channel required for vertebrate sensory hair cell mechanotransduction". In: Science 301.5629, pages 96-99. 
Simpson, S. (2011). Advances in Insect Physiology. Advances in Insect Physiology Bd. 33. Elsevier Science. ISBN: 9780080471174. URL: https : //books . google .de/books? id $=\mathrm{D} \% 5 \mathrm{C}_{-}$YlngKw3bUC.

Sokabe, Takaaki, Hsiang-Chin Chen, Junjie Luo, and Craig Montell (2016). "A switch in thermal preference in Drosophila larvae depends on multiple rhodopsins". In: Cell reports 17.2, pages 336-344.

Sokabe, Takaaki, Seiya Tsujiuchi, Tatsuhiko Kadowaki, and Makoto Tominaga (2008). "Drosophila painless is a Ca2+-requiring channel activated by noxious heat". In: Journal of Neuroscience 28.40, pages 9929-9938.

Standen, NB and PR Stanfield (1982). "A binding-site model for calcium channel inactivation that depends on calcium entry". In: Proceedings of the Royal Society of London. Series B. Biological Sciences 217.1206, pages 101-110.

Steinbrecht, Rudolf Alexander (1989). "The fine structure of thermo-/hygrosensitive sensilla in the silkmoth Bombyx mori: receptor membrane substructure and sensory cell contacts". In: Cell and Tissue Research 255.1, pages 49-57.

Steinmetz, Janina and Ann-Christin Posten (2017). "Physical temperature affects response behavior". In: Journal of Experimental Social Psychology 70, pages 294-300.

Stevenson, RD (1985). "The relative importance of behavioral and physiological adjustments controlling body temperature in terrestrial ectotherms". In: The American Naturalist 126.3, pages 362-386.

Stocker, RF, MC Lienhard, A Borst, and KF Fischbach (1990). "Neuronal architecture of the antennal lobe in Drosophila melanogaster". In: Cell and tissue research 262.1, pages 9-34.

Sukharev, Sergei I, Paul Blount, Boris Martinac, Frederick R Blattner, and Ching Kung (1994). "A large-conductance mechanosensitive channel in $E$. coli encoded by $\mathrm{mscL}$ alone". In: Nature 368.6468, page 265.

Sun, Yishan, Lei Liu, Yehuda Ben-Shahar, Julie S Jacobs, Daniel F Eberl, and Michael J Welsh (2009). "TRPA channels distinguish gravity sensing from hearing in Johnston's organ". In: Proceedings of the National Academy of Sciences 106.32, pages 13606-13611.

Takahashi, Nobuaki, Yusuke Mizuno, Daisuke Kozai, Shinichiro Yamamoto, Shigeki Kiyonaka, Takahiro Shibata, Koji Uchida, and Yasuo Mori (2008). "Molecular characterization of TRPA1 channel activation by cysteine-reactive inflammatory mediators". In: Channels 2.4, pages 287-298.

Tang, Xin, Michael D Platt, Christopher M Lagnese, Jennifer R Leslie, and Fumika N Hamada (2013). "Temperature integration at the AC thermosensory neurons in Drosophila". In: Journal of Neuroscience 33.3, pages 894-901.

Thibault, Stephen T, Matthew A Singer, Wesley Y Miyazaki, Brett Milash, Nicholas A Dompe, Carol M Singh, Ross Buchholz, Madelyn Demsky, Robert Fawcett, Helen L 
Francis-Lang, et al. (2004). "A complementary transposon tool kit for Drosophila melanogaster using P and piggyBac". In: Nature genetics 36.3, page 283.

Todi, Sokol V, Yashoda Sharma, and Daniel F Eberl (2004). "Anatomical and molecular design of the Drosophila antenna as a flagellar auditory organ". In: Microscopy research and technique 63.6, pages 388-399.

Tracey Jr, W Daniel, Rachel I Wilson, Gilles Laurent, and Seymour Benzer (2003). "painless, a Drosophila gene essential for nociception". In: Cell 113.2, pages 261-273.

Tsubouchi, Asako, Jason C Caldwell, and W Daniel Tracey (2012). "Dendritic filopodia, Ripped Pocket, NOMPC, and NMDARs contribute to the sense of touch in Drosophila larvae". In: Current Biology 22.22, pages 2124-2134.

Tu, Yuhai (2013). "Quantitative modeling of bacterial chemotaxis: signal amplification and accurate adaptation". In: Annual review of biophysics 42, pages 337-359.

Turner, Heather N, Kevin Armengol, Atit A Patel, Nathaniel J Himmel, Luis Sullivan, Srividya Chandramouli lyer, Surajit Bhattacharya, Eswar Prasad R lyer, Christian Landry, Michael J Galko, and Daniel N Cox (2016). "The TRP channels Pkd2, NompC, and Trpm act in cold-sensing neurons to mediate unique aversive behaviors to noxious cold in Drosophila". In: Current Biology 26.23, pages 3116-3128.

Ursell, Tristan, Kerwyn Casey Huang, Eric Peterson, and Rob Phillips (2007). "Cooperative gating and spatial organization of membrane proteins through elastic interactions". In: PLoS computational biology 3.5, e81.

Valdez, Francisco J Sierra, Caleigh M Azumaya, Luis O Romero, Terunaga Nakagawa, and Julio F Cordero-Morales (2019). "Structural and Functional Analyses of TRPC3 Reveal Allosteric Gating Modulation by the Cytoplasmic Domain". In: Biophysical Journal 116.3, 451a.

Vay, Laura, Chunjing Gu, and Peter A McNaughton (2012). "The thermo-TRP ion channel family: properties and therapeutic implications". In: British journal of pharmacology 165.4, pages 787-801.

Venkatachalam, Kartik and Craig Montell (2007). "TRP channels". In: Annu. Rev. Biochem. 76, pages 387-417.

Voets, Thomas (2012). "Quantifying and modeling the temperature-dependent gating of TRP channels". In: Reviews of physiology, biochemistry and pharmacology. Springer, pages 91-119.

Voets, Thomas, Guy Droogmans, Ulrich Wissenbach, Annelies Janssens, Veit Flockerzi, and Bernd Nilius (2004). "The principle of temperature-dependent gating in cold-and heat-sensitive TRP channels". In: Nature 430.7001, page 748.

Walker, Richard G, Aarron T Willingham, and Charles S Zuker (2000). "A Drosophila mechanosensory transduction channel". In: Science 287.5461, pages 2229-2234.

Wang, Qi, Bo Shui, Michael I Kotlikoff, and Holger Sondermann (2008). "Structural basis for calcium sensing by GCaMP2". In: Structure 16.12, pages 1817-1827. 
Waring, Alan (1996). Practical systems thinking. Cengage Learning EMEA.

Wark, Barry, Brian Nils Lundstrom, and Adrienne Fairhall (2007). "Sensory adaptation". In: Current opinion in neurobiology 17.4, pages 423-429.

Watnick, Terry J, Ying Jin, Erika Matunis, Maurice J Kernan, and Craig Montell (2003). "A flagellar polycystin-2 homolog required for male fertility in Drosophila". In: Current Biology 13.24, pages 2179-2184.

White, Kristin, Megan E Grether, John M Abrams, Lynn Young, Kim Farrell, and Hermann Steller (1994). "Genetic control of programmed cell death in Drosophila". In: Science 264.5159, pages 677-683.

Wilkinson, Miles F (2019). Genetic paradox explained by nonsense.

Wong, Ching-On, Kuchuan Chen, Yong Qi Lin, Yufang Chao, Lita Duraine, Zhongmin Lu, Wan Hee Yoon, Jeremy M Sullivan, Geoffrey T Broadhead, Charlotte J Sumner, et al. (2014). "A TRPV channel in Drosophila motor neurons regulates presynaptic resting Ca2+ levels, synapse growth, and synaptic transmission". In: Neuron 84.4, pages 764777.

Wong, Ching-On, Ruoxia Li, Craig Montell, and Kartik Venkatachalam (2012). "Drosophila TRPML is required for TORC1 activation". In: Current Biology 22.17, pages 1616-1621.

Wong, Ching-On, Michela Palmieri, Jiaxing Li, Dmitry Akhmedov, Yufang Chao, Geoffrey T Broadhead, Michael X Zhu, Rebecca Berdeaux, Catherine A Collins, Marco Sardiello, et al. (2015). "Diminished MTORC1-dependent JNK activation underlies the neurodevelopmental defects associated with lysosomal dysfunction". In: Cell reports 12.12, pages 2009-2020.

Xiao, Fuyuan and Bowen Qin (2018). "A weighted combination method for conflicting evidence in multi-sensor data fusion". In: Sensors 18.5, page 1487.

Xu, SY, CL Cang, XF Liu, YQ Peng, YZ Ye, ZQ Zhao, and AK Guo (2006). "Thermal nociception in adult Drosophila: behavioral characterization and the role of the painless gene". In: Genes, Brain and Behavior 5.8, pages 602-613.

Yan, Zhiqiang, Wei Zhang, Ye He, David Gorczyca, Yang Xiang, Li E Cheng, Shan Meltzer, Lily Yeh Jan, and Yuh Nung Jan (2013). "Drosophila NOMPC is a mechanotransduction channel subunit for gentle-touch sensation". In: Nature 493.7431, page 221.

Yang, Fan, Yuanyuan Cui, KeWei Wang, and Jie Zheng (2010). "Thermosensitive TRP channel pore turret is part of the temperature activation pathway". In: Proceedings of the National Academy of Sciences 107.15, pages 7083-7088.

Zanini, Damiano, Diego Giraldo, Ben Warren, Radoslaw Katana, Marta Andrés, Suneel Reddy, Stephanie Pauls, Nicola Schwedhelm-Domeyer, Bart RH Geurten, and Martin C Göpfert (2018). "Proprioceptive opsin functions in Drosophila larval locomotion". In: Neuron 98.1, pages 67-74. 
Zhang, Mingfeng, Xia Li, Honglan Zheng, Xiaoxu Wen, Sihan Chen, Jia Ye, Siyang Tang, Fuqiang Yao, Yuezhou Li, and Zhiqiang Yan (2018). "Brv1 is required for Drosophila larvae to sense gentle touch". In: Cell reports 23.1, pages 23-31.

Zhang, Wei, Li E Cheng, Maike Kittelmann, Jiefu Li, Maja Petkovic, Tong Cheng, Peng Jin, Zhenhao Guo, Martin C Göpfert, Lily Yeh Jan, et al. (2015). "Ankyrin repeats convey force to gate the NOMPC mechanotransduction channel". In: Cell 162.6, pages 13911403.

Zhang, Wei, Zhiqiang Yan, Lily Yeh Jan, and Yuh Nung Jan (2013). "Sound response mediated by the TRP channels NOMPC, NANCHUNG, and INACTIVE in chordotonal organs of Drosophila larvae". In: Proceedings of the National Academy of Sciences 110.33, pages 13612-13617.

Zheng, Wenjun and Han Wen (2019). "Heat activation mechanism of TRPV1: New insights from molecular dynamics simulation". In: Temperature, pages 1-12.

Zhong, Lixian, Andrew Bellemer, Haidun Yan, Ken Honjo, Jessica Robertson, Richard Y Hwang, Geoffrey S Pitt, and W Daniel Tracey (2012). "Thermosensory and nonthermosensory isoforms of Drosophila melanogaster TRPA1 reveal heat-sensor domains of a thermoTRP Channel". In: Cell reports 1.1, pages 43-55.

Zhou, Yao, Li-Hui Cao, Xiu-Wen Sui, Xiao-Qing Guo, and Dong-Gen Luo (2019). "Mechanosensory circuits coordinate two opposing motor actions in Drosophila feeding". In: Science advances 5.5, eaaw5141. 
Chapter 6

Appendix 


\section{List of Appendix Figures}

A1 $\mathrm{Ca}^{2+}$ responses compiled to temperature stimulus (Descending Amplitude, first step hot $\ldots \ldots \ldots$. . . . . . . . . . . . . . . 94

A2 $\mathrm{Ca}^{2+}$ responses compiled to temperature stimulus (Descending Amplitude, first step cold $\ldots \ldots \ldots$. . . . . . . . . . . . . 95

A3 $\mathrm{Ca}^{2+}$ responses compiled to temperature Stimulus (Ascending Amplitude,

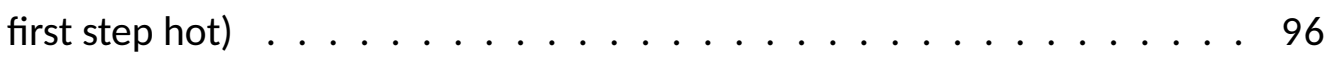

A4 $\mathrm{Ca}^{2+}$ responses compiled to temperature stimulus (Ascending Amplitude, first step cold $\ldots \ldots \ldots$. . . . . . . . . . . . . . 97

A5 $\mathrm{Ca}^{2+}$ responses of CantonS flies to different temperature step stimuli . . . 98

A6 $\mathrm{Ca}^{2+}$ responses of $w^{1118}$ flies to different temperature step stimuli . . . . 99

A7 $\mathrm{Ca}^{2+}$ responses of nompC $\mathrm{C}^{3}$ flies to different temperature step stimuli . . . 100

A8 $\mathrm{Ca}^{2+}$ responses of nompC $\mathrm{C}^{3} /+$ flies to different temperature step stimuli . . 101

A9 $\mathrm{Ca}^{2+}$ responses of nompC-rescue flies to different temperature step stimuli 102

A10 $\mathrm{Ca}^{2+}$ responses of nomp $\mathrm{C}^{\text {f00914 }}$ flies to different temperature step stimuli . 103

A11 $\mathrm{Ca}^{2+}$ responses of nompC-overexpression flies to different temperature step stimuli . . . . . . . . . . . . . . . . . . . 104

A12 $\mathrm{Ca}^{2+}$ responses of nompC-Gal4 and UAS-nompC control flies . . . . . 105 
6.1 Appendix: $\mathrm{Ca}^{2+}$ traces sorted by temperature stimulus

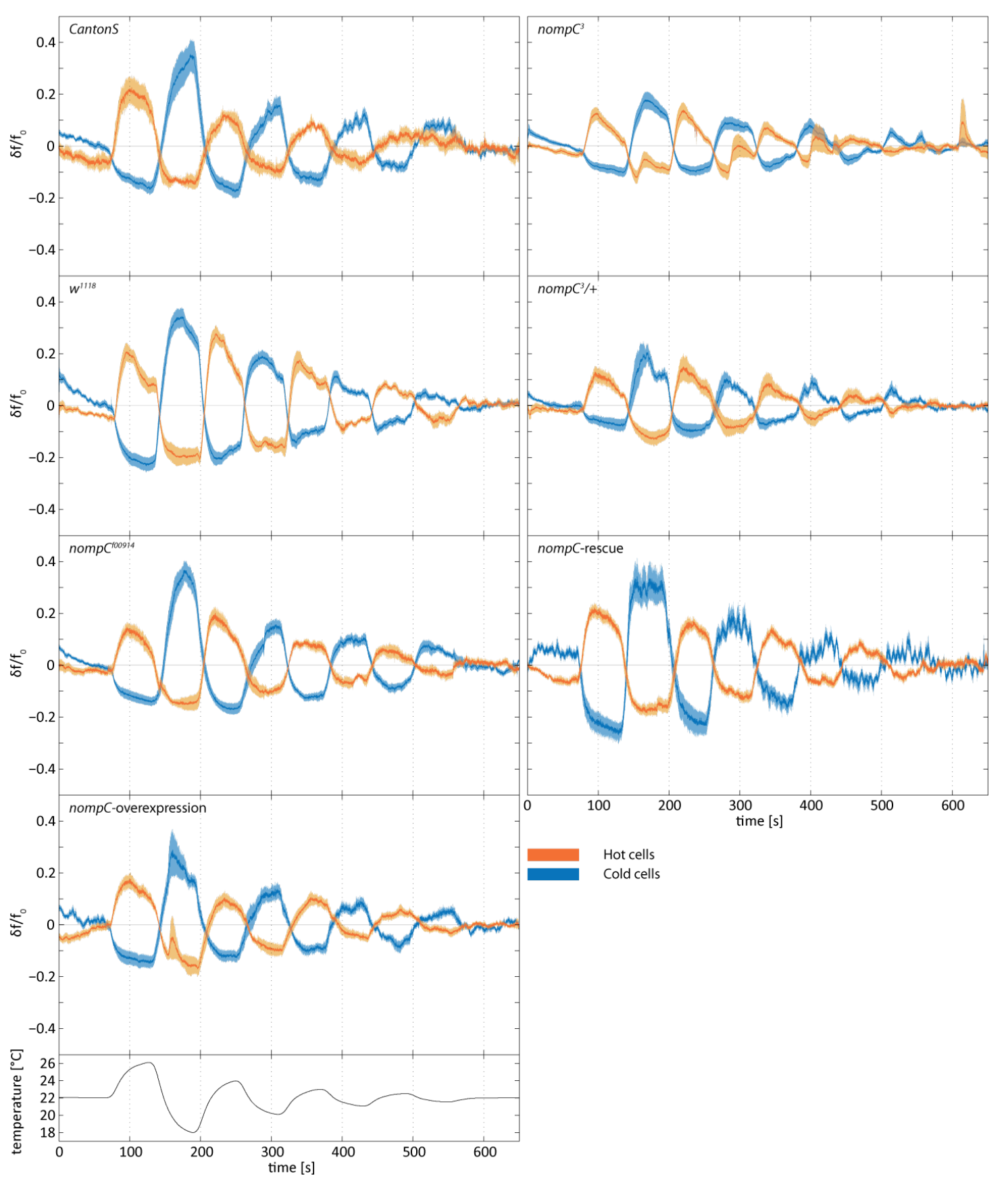

Appendix Figure $\mathrm{A} 1: \mathrm{Ca}^{2+}$ responses compiled to temperature stimulus (Descending Amplitude, first step hot) Overview of $\Delta \mathrm{F} / \mathrm{F}_{0}$ responses of fly lines used in this study to termperature stimulation. Temperature stimulus shown in lower right. Temperature starts at $22^{\circ} \mathrm{C}$ baseline. Order of temperature steps: $26^{\circ} \mathrm{C}, 18^{\circ} \mathrm{C}, 24^{\circ} \mathrm{C}, 20^{\circ} \mathrm{C}, 23^{\circ} \mathrm{C}, 21^{\circ} \mathrm{C}, 22.5^{\circ} \mathrm{C}$, $21.5^{\circ} \mathrm{C}$, then return to $22^{\circ} \mathrm{C}$ baseline. $\Delta \mathrm{F} / \mathrm{F}_{\mathrm{O}}$ curves show mean value as solid line, $\mathrm{SEM}$ as shaded area. $\mathrm{HC}$ signals in orange, $\mathrm{CC}$ signals in blue.

n: Cantons: $4 \mathrm{HCs}, 19 \mathrm{CCs}, \mathrm{w}^{1118}$ : $10 \mathrm{HCs}, 13 \mathrm{CCs}$, nompC f00914: $6 \mathrm{HCs}, 10 \mathrm{CCs}$,nompCoverexpression: $5 \mathrm{HCs}, 8 \mathrm{CCs}$,nompC $\mathrm{C}^{3}: 11 \mathrm{HCs}, 15 \mathrm{CCs}_{\text {, nompC }}^{3} / \mathrm{t}$ : $4 \mathrm{HCs}, 4 \mathrm{CCs}$, nompCrescue: $8 \mathrm{HCs}, 5 \mathrm{CCs}$

94 


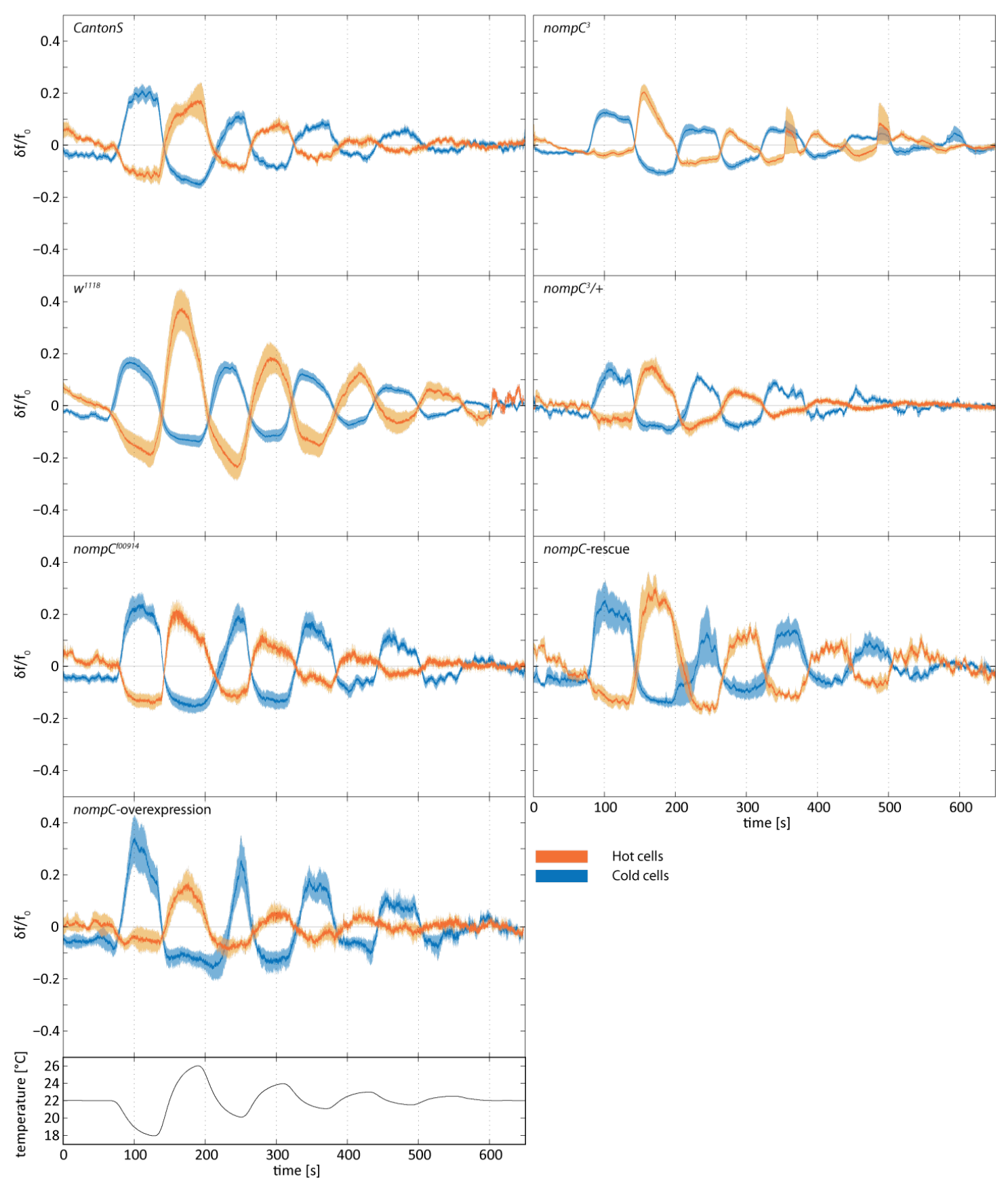

Appendix Figure A2: $\mathrm{Ca}^{2+}$ responses to Temperature Stimuli (Descending Amplitude, first step cold) Overview of $\Delta \mathrm{F} / \mathrm{F}_{\mathrm{O}}$ responses of fly lines used in this study to temperatore stimulation. Temperature stimulus shown in lower right. Temperature starts at $22^{\circ} \mathrm{C}$ baseline. Order of temperature steps: $18^{\circ} \mathrm{C}, 26^{\circ} \mathrm{C}, 20^{\circ} \mathrm{C}, 24^{\circ} \mathrm{C}, 21^{\circ} \mathrm{C}, 23^{\circ} \mathrm{C}, 21.5^{\circ} \mathrm{C}, 22.5^{\circ} \mathrm{C}$, then return to $22^{\circ} \mathrm{C}$ baseline. $\Delta \mathrm{F} / \mathrm{F}_{0}$ curves show mean value as solid line, SEM as shaded area. $\mathrm{HC}$ signals in orange, $\mathrm{CC}$ signals in blue.

n: Cantons: $6 \mathrm{HCs}, 13 \mathrm{CCs}, w^{1118}$ : $5 \mathrm{HCs}, 11 \mathrm{CCs}$,nompC f00914: $10 \mathrm{HCs}, 14 \mathrm{CCs}$,nompCoverexpression: $4 \mathrm{HCs}, 5 \mathrm{CCs}$, nompC ${ }^{3}$ : $11 \mathrm{HCs}, 12 \mathrm{CCs}_{\text {, nompC }}^{3} /+$ : $4 \mathrm{HCs}, 6 \mathrm{CCs}$, nompCrescue: $5 \mathrm{HCs}, 4 \mathrm{CCs}$

95 


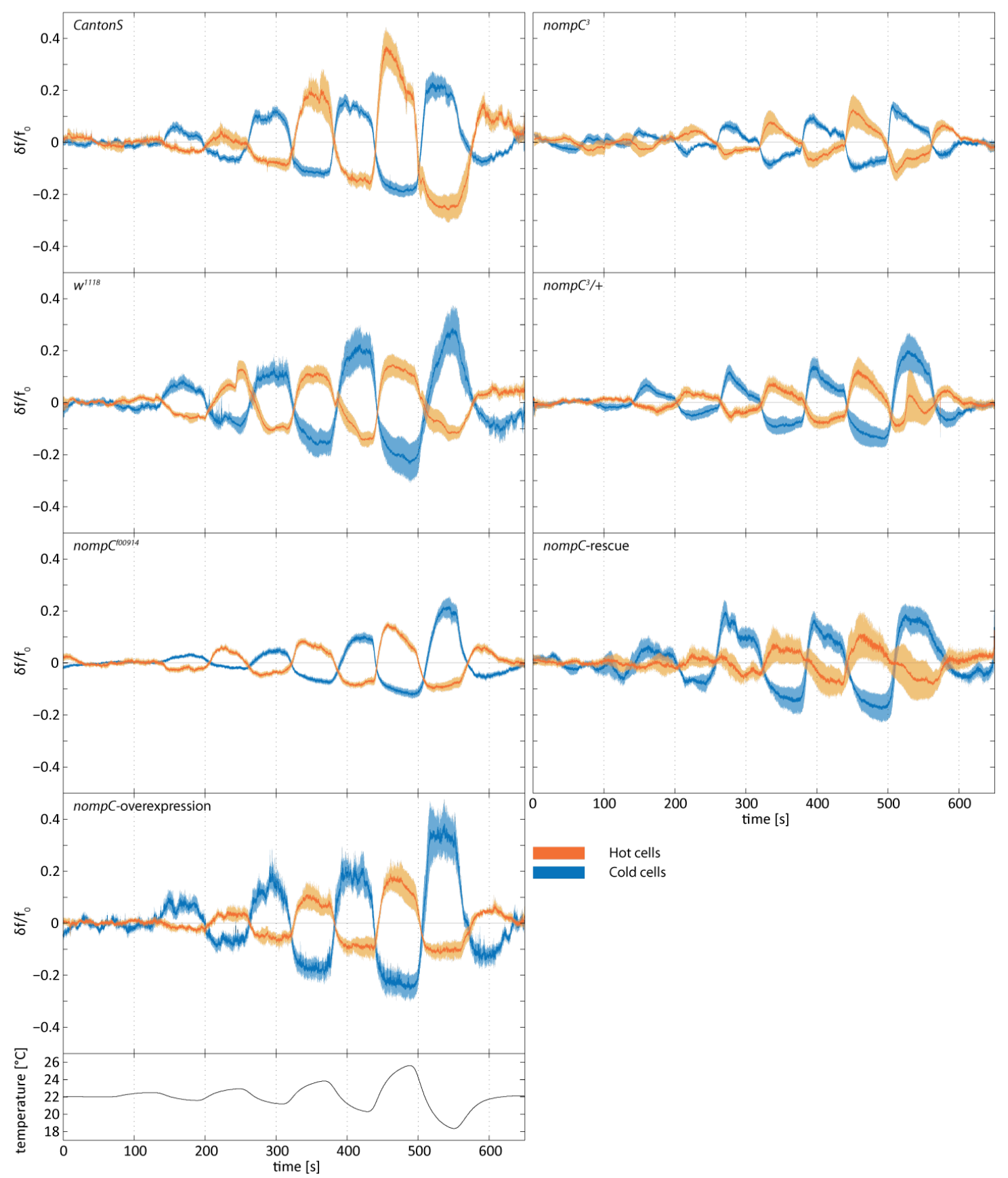

Appendix Figure $\mathrm{A3}: \mathrm{Ca}^{2+}$ responses compiled to temperature stimulus (Ascending Amplitude, first step hot) Overview of $\Delta \mathrm{F} / \mathrm{F}_{\mathrm{O}}$ responses of fly lines used in this study to temperature stimulation. Temperature stimulus shown in lower right. Temperature starts at $22^{\circ} \mathrm{C}$ baseline. Order of temperature steps: $22.5^{\circ} \mathrm{C}, 21.5^{\circ} \mathrm{C}, 23^{\circ} \mathrm{C}, 21^{\circ} \mathrm{C}, 24^{\circ} \mathrm{C}, 20^{\circ} \mathrm{C}, 26^{\circ} \mathrm{C}$, $18^{\circ} \mathrm{C}$, then return to $22^{\circ} \mathrm{C}$ baseline. $\Delta \mathrm{F} / \mathrm{F}_{0}$ curves show mean value as solid line, $\mathrm{SEM}$ as shaded area. $\mathrm{HC}$ signals in orange, $\mathrm{CC}$ signals in blue.

n: Cantons: $6 \mathrm{HCs}, 12 \mathrm{CCs}, w^{1118}$ : $12 \mathrm{HCs}, 7 \mathrm{CCs}$,nompC f00914: $6 \mathrm{HCs}, 11 \mathrm{CCs}$,nompCoverexpression: $4 \mathrm{HCs}, 7 \mathrm{CCs}$, nompC $\mathrm{m}^{3}$ : $8 \mathrm{HCs}, 5 \mathrm{CCs}_{\text {, nompC }}^{3} /+$ : $7 \mathrm{HCs}, 10 \mathrm{CCs}$, nompCrescue: $4 \mathrm{HCs}, 4 \mathrm{CCs}$ 


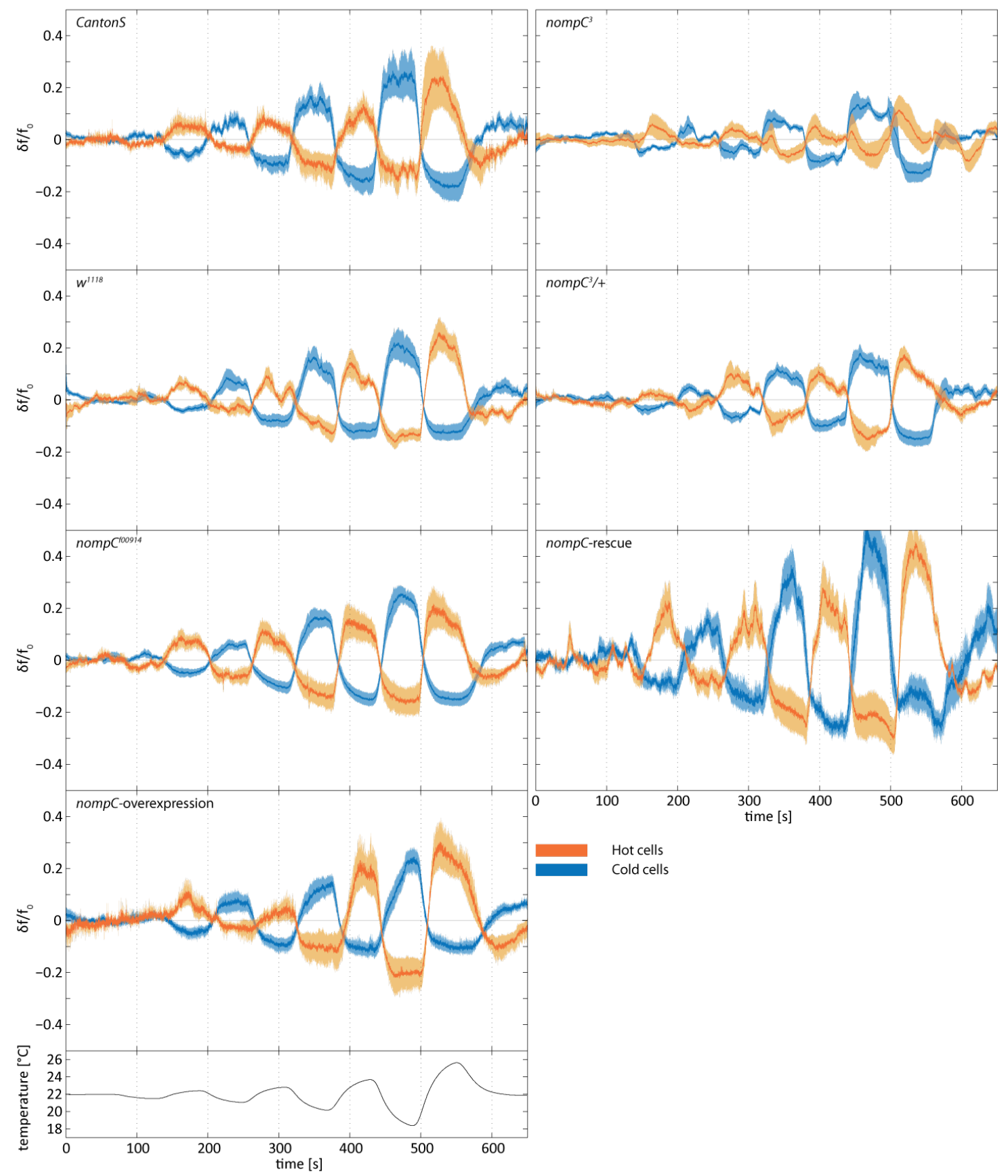

Appendix Figure A4: $\mathrm{Ca}^{2+}$ responses compiled to temperature stimulus (Ascending Amplitude, first step cold) Overview of $\Delta F / F_{0}$ responses of fly lines used in this study to temperature stimulation. Temperature stimulus shown in lower right. Temperature starts at $22^{\circ} \mathrm{C}$ baseline. Order of temperature steps: $21.5^{\circ} \mathrm{C}, 22.5^{\circ} \mathrm{C}, 21^{\circ} \mathrm{C}, 23^{\circ} \mathrm{C}, 20^{\circ} \mathrm{C}, 24^{\circ} \mathrm{C}, 18^{\circ} \mathrm{C}$, $26^{\circ} \mathrm{C}$, then return to $22^{\circ} \mathrm{C}$ baseline. $\Delta \mathrm{F} / \mathrm{F}_{0}$ curves show mean value as solid line, SEM as shaded area. $\mathrm{HC}$ signals in orange, $\mathrm{CC}$ signals in blue.

$\mathrm{n}$ : Cantons: $4 \mathrm{HCs}, 10 \mathrm{CCs}, \mathrm{w}^{1118}$ : $5 \mathrm{HCs}, 10 \mathrm{CCs}$,nompC f00914: $5 \mathrm{HCs}, 12 \mathrm{CCs}$,nompCoverexpression: $5 \mathrm{HCs}, 6 \mathrm{CCs}$, nompC $^{3}$ : $8 \mathrm{HCs}, 5 \mathrm{CCs}_{\text {, nompC }}^{3} /+$ : $6 \mathrm{HCs}, 8 \mathrm{CCs}$, nompCrescue: $4 \mathrm{HCs}, 6 \mathrm{CCs}$ 
6.2 Appendix: $\mathrm{Ca}^{2+}$ traces sorted by fly strains

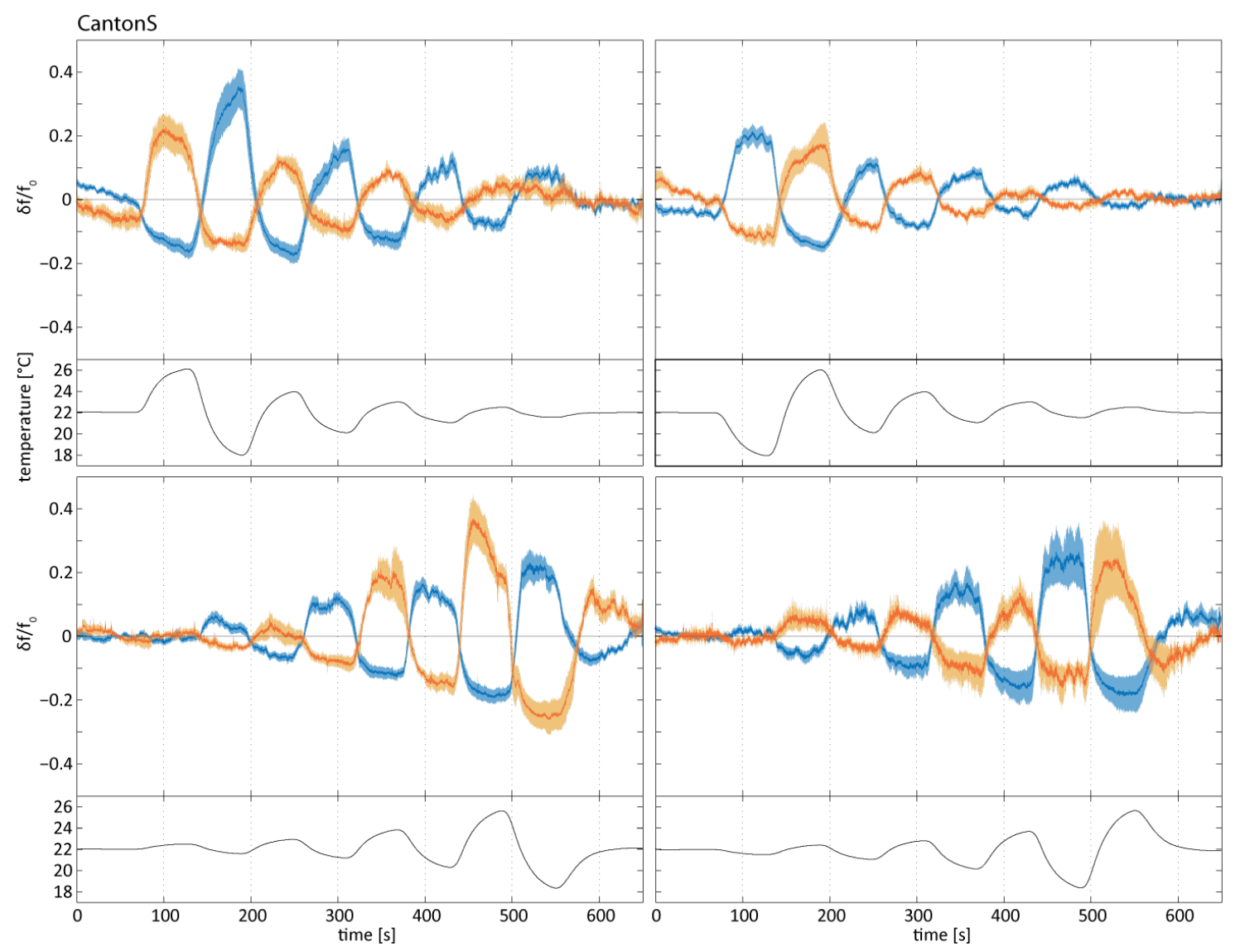

Appendix Figure A5: $\mathrm{Ca}^{2+}$ responses of CantonS flies to different temperature step simuni Overview of $\Delta F / F_{0}$ responses of Cantons flies to the temperature step stimulus photocols. Solid lanes in $\mathrm{Ca}^{2+}$ traces denote mean response, shaded areas are respective SEM. HCs shown in orange, COs shown in blue.

98 


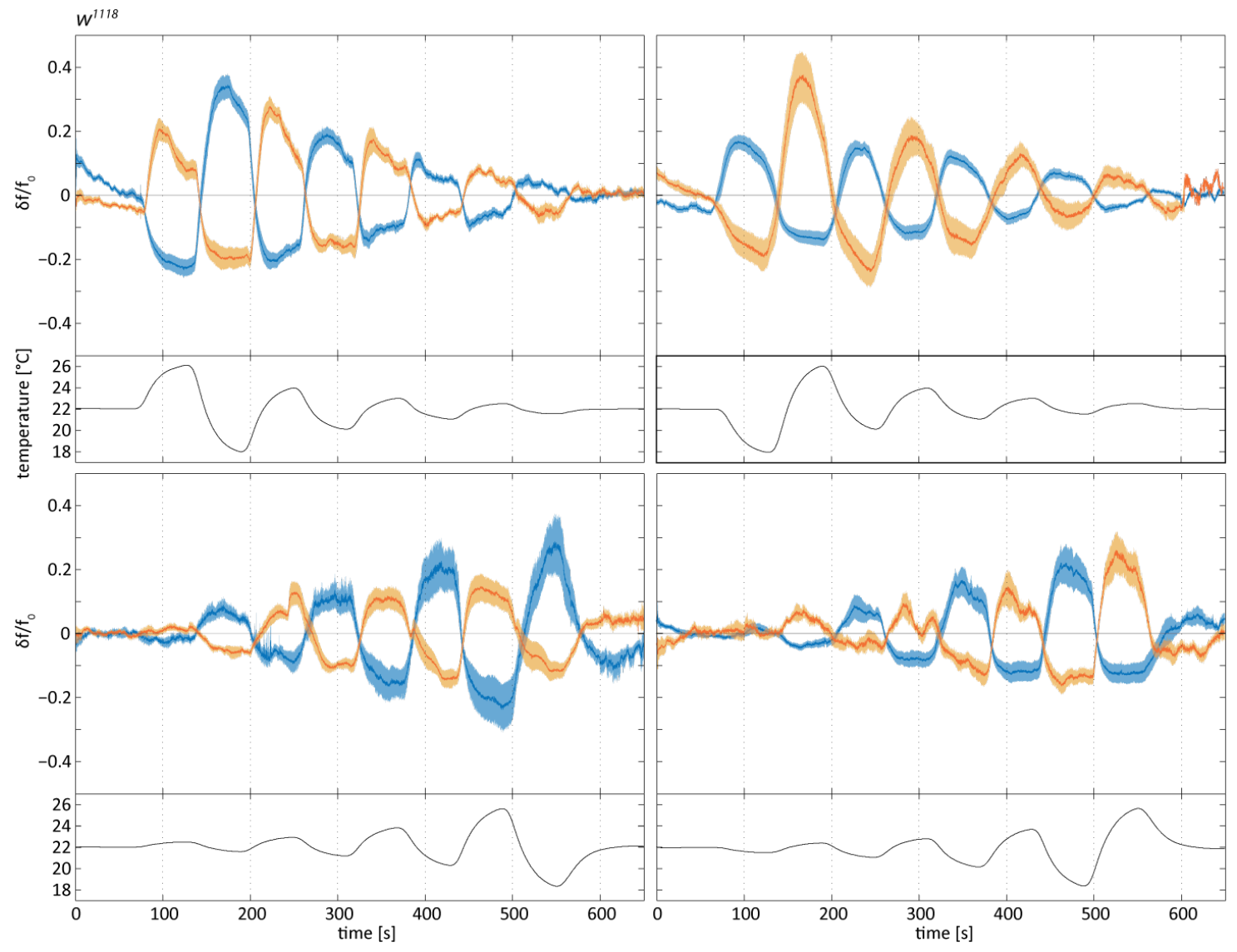

Appendix Figure $\mathrm{A6}$ : $\mathrm{Ca}^{2+}$ responses of $w^{1118}$ flies to different temperature step stimuli Overview of $\Delta F / F_{0}$ responses of $W^{1118}$ flies to the temperature step stimulus protocols. Solid lanes in $\mathrm{Ca}^{2+}$ traces denote mean response, shaded areas are respective SEM. HCs shown in orange, CCs shown in blue. 


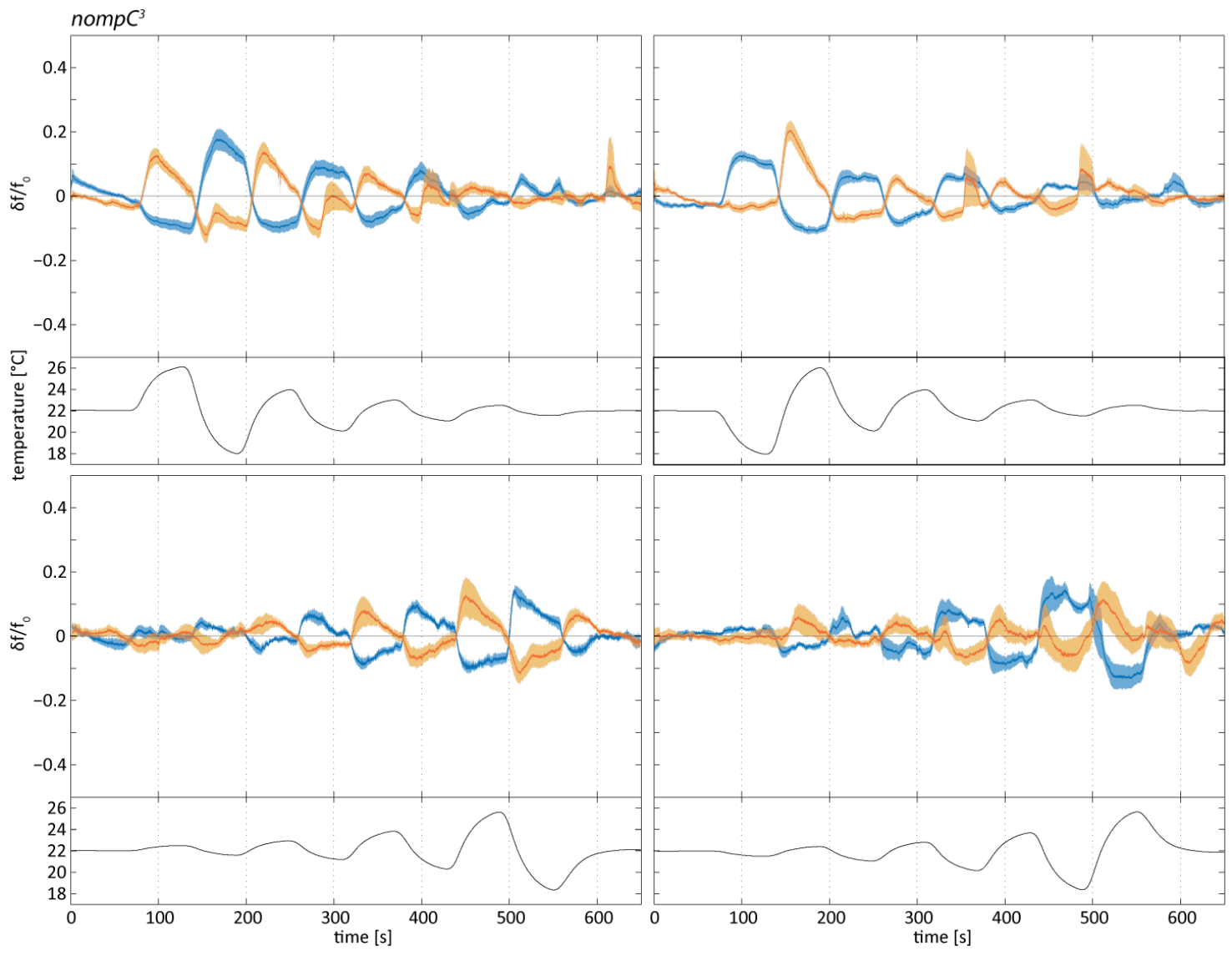

Appendix Figure A7: $\mathrm{Ca}^{2+}$ responses of nompC ${ }^{3}$ flies to different temperature step stimuli Overview of $\triangle \mathrm{F} / \mathrm{F}_{0}$ responses of nompC $\mathrm{C}^{3}$ flies to the temperature step stimulus protocols. Solid lanes in $\mathrm{Ca}^{2+}$ traces denote mean response, shaded areas are respective SEM. HCs shown in orange, CCs shown in blue. 


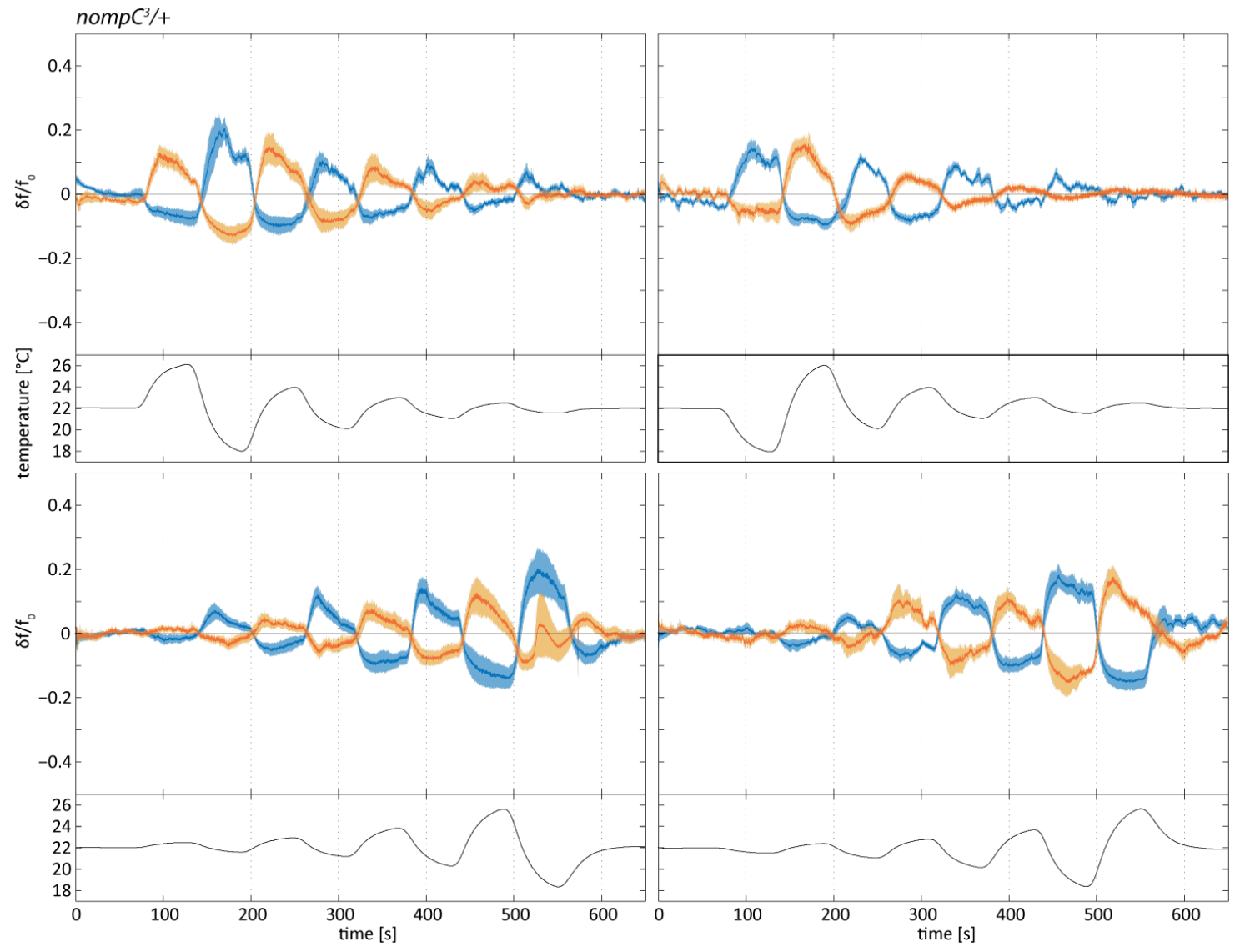

Appendix Figure A8: $\mathrm{Ca}^{2+}$ responses of nompC $\mathrm{C}^{3} /+$ flies to different temperature step stimuli Overview of $\Delta \mathrm{F} / \mathrm{F}_{0}$ responses of nompC $\mathrm{C}^{3} /+$ flies to the temperature step stimulus protocols. Solid lanes in $\mathrm{Ca}^{2+}$ traces denote mean response, shaded areas are respective SEM. HCs shown in orange, CCs shown in blue. 


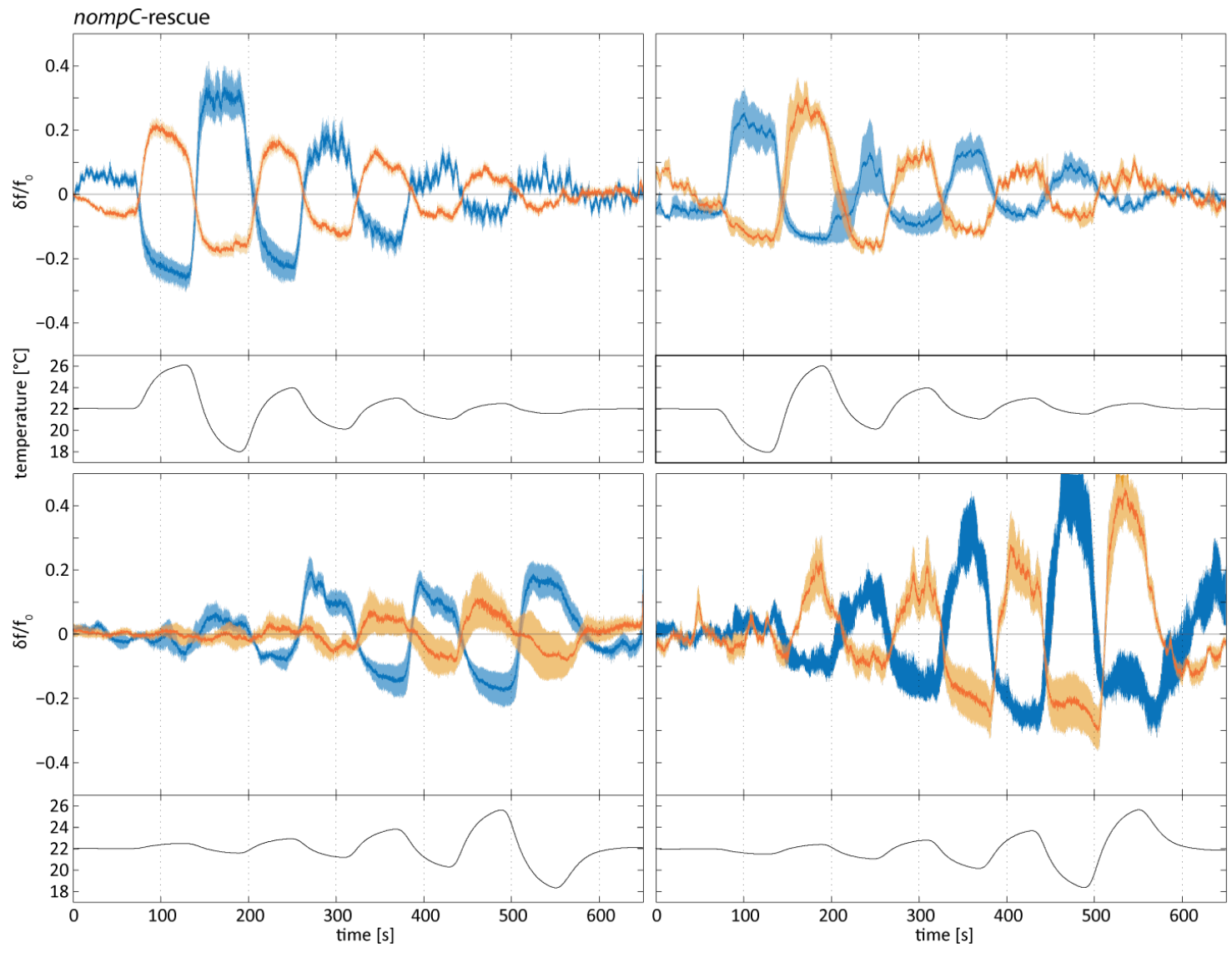

Appendix Figure A9: $\mathrm{Ca}^{2+}$ responses of nompC-rescue flies to different temperature step stimuli Overview of $\Delta F / F_{0}$ responses of nompC-rescue flies to the temperature step stimulus protocols. Solid lanes in $\mathrm{Ca}^{2+}$ traces denote mean response, shaded areas are respective SEM. HCs shown in orange, CCs shown in blue. 


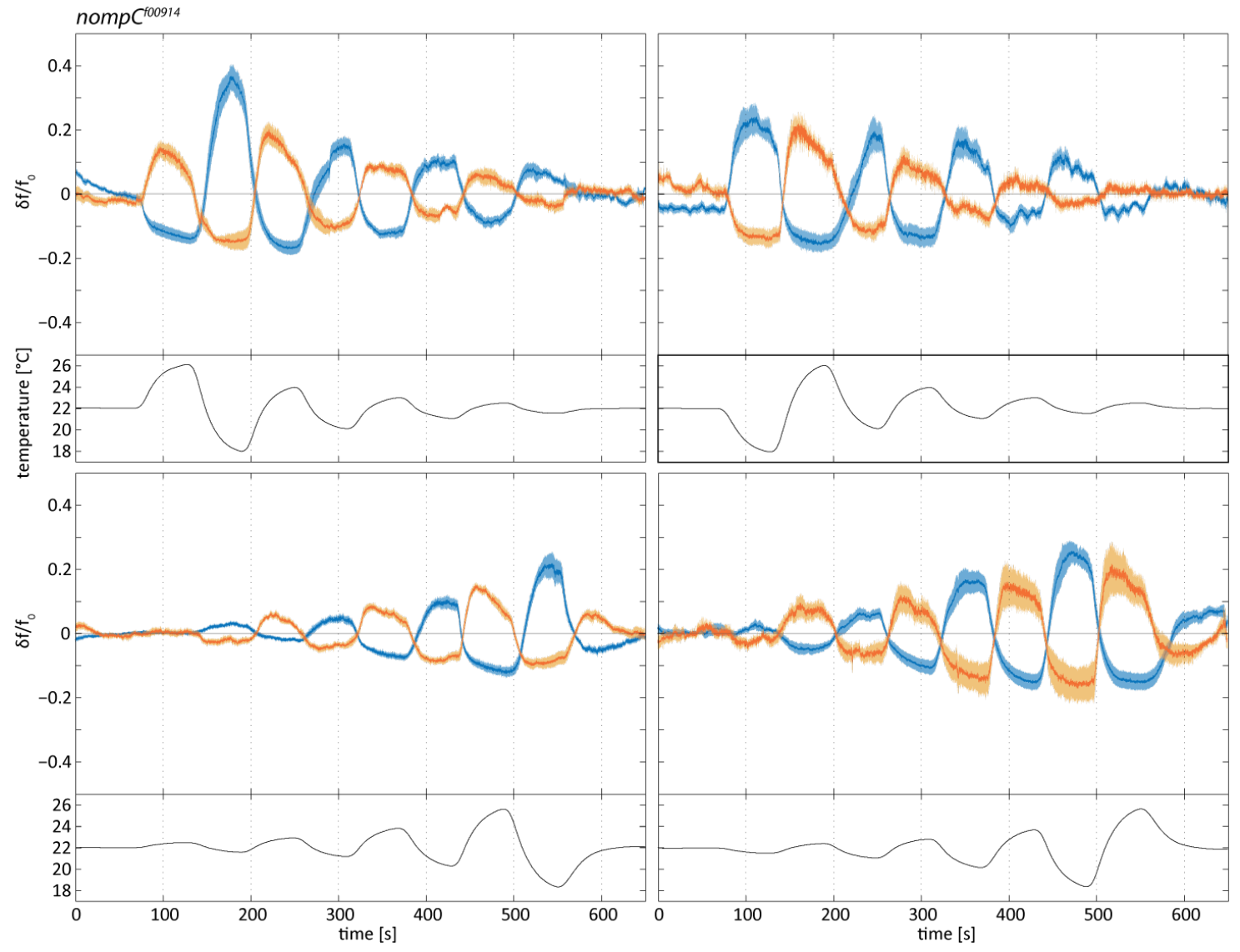

Appendix Figure A10: $\mathrm{Ca}^{2+}$ responses of nomp $\mathrm{C}^{\text {f00914 }}$ flies to different temperature step stimuli Overview of $\Delta F / F_{0}$ responses of nompC $C^{f 00914}$ flies to the temperature step stimulus protocols. Solid lanes in $\mathrm{Ca}^{2+}$ traces denote mean response, shaded areas are respective SEM. HCs shown in orange, CCs shown in blue. 


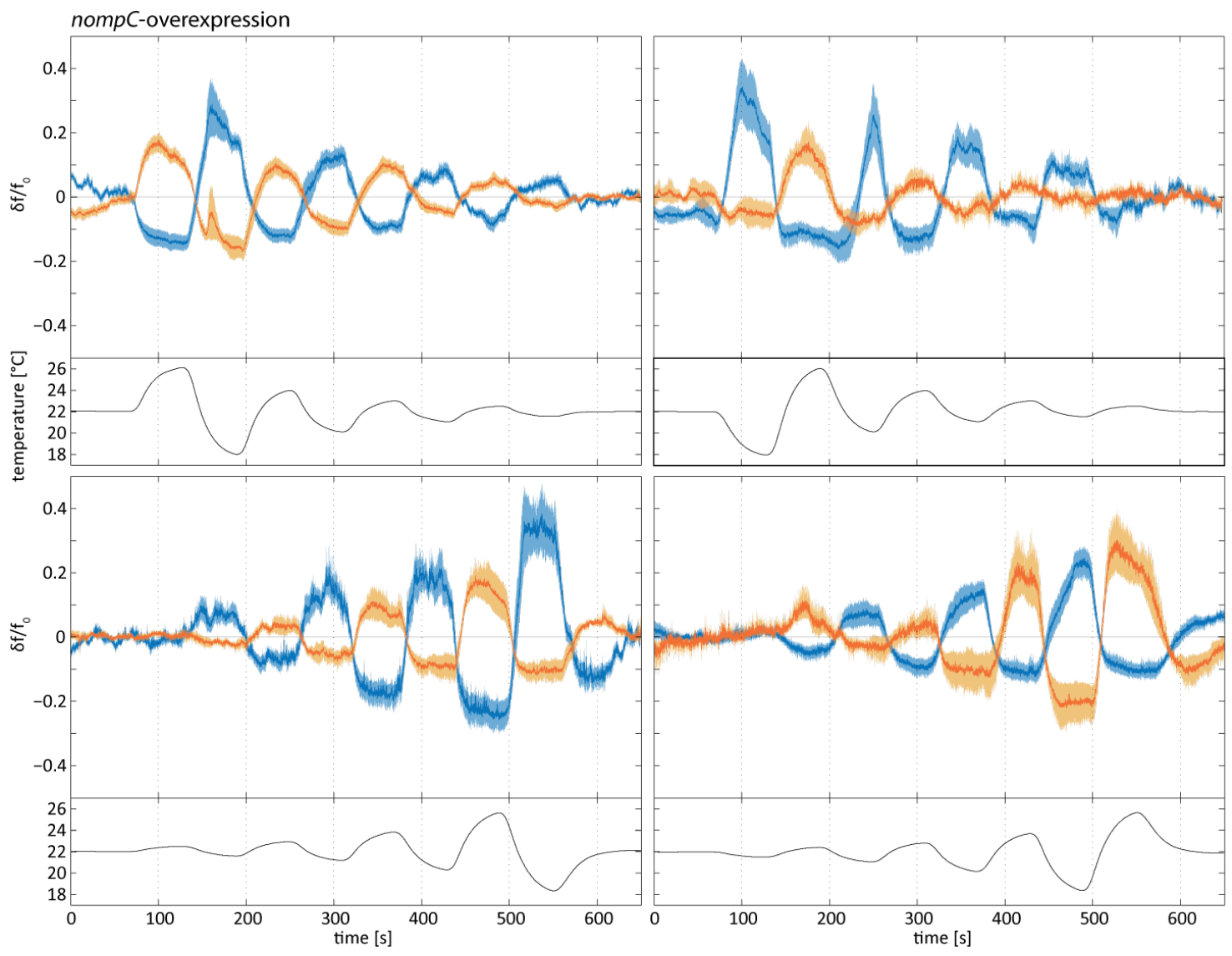

Appendix Figure A11: $\mathrm{Ca}^{2+}$ responses of nompC-overexpression flies to different temperature step stimuli Overview of $\Delta \mathrm{F} / \mathrm{F}_{0}$ responses of nompC-overexpression flies to the temperature step stimulus protocols. Solid lanes in $\mathrm{Ca}^{2+}$ traces denote mean response, shaded areas are respective SEM. HCs shown in orange, CCs shown in blue. 

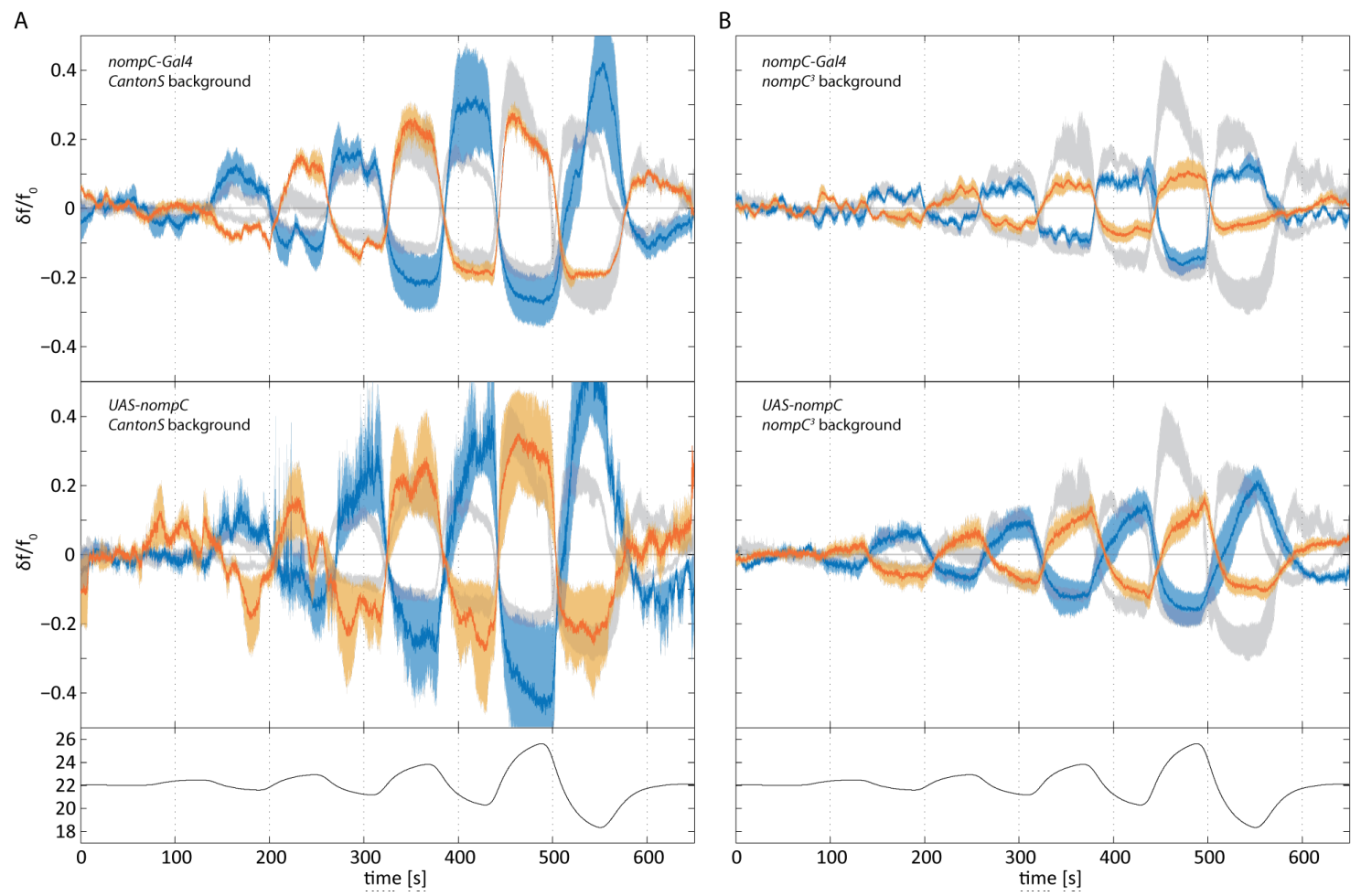

Appendix Figure A12: $\mathrm{CCa}^{2+}$ responses of nompC-Gal4 and UAS-nompC control flies Overview of $\triangle \mathrm{F} / \mathrm{F}_{0}$ responses of Gal4 and UAS control fly lines in both the wildtype and homozygous nomp $\mathrm{C}^{3}$ background. Temperature stimuli on bottom line. Temperature starts at $22^{\circ} \mathrm{C}$ baseline. Order of temperature steps: $22.5^{\circ} \mathrm{C}, 21.5^{\circ} \mathrm{C}, 23^{\circ} \mathrm{C}, 21^{\circ} \mathrm{C}, 24^{\circ} \mathrm{C}$, $20^{\circ} \mathrm{C}, 26^{\circ} \mathrm{C}, 18^{\circ} \mathrm{C}$, then return to $22^{\circ} \mathrm{C}$ baseline. $\Delta \mathrm{F} / \mathrm{F}_{0}$ curves show mean value as solid line, SEM as shaded area. HC signals in orange, CC signals in blue. Shaded grey area shows the CantonS responses SEM during the same stimulus condition (See Appendixfigures A5 and A3). A) Responses of nompC-Gal4 (top) and UAS-nompC (middle) control flies in the CantonS wildtype background. HC responses do not differ greatly from Cantons flies for either line. B) Responses of nompC-Gal4 (top) and UAS-nompC (middle) control flies in the nompC $\mathrm{C}^{3}$ mutant background. $\mathrm{HC}$ response amplitude is reduced in both lines compared to wildtype controls, as would be exprected from nompC null mutants from previous experiments.

n: nompC-Gal4, wildtype background: $5 \mathrm{HCs}, 6 \mathrm{CCs}$,UAS-nompC wildtype background: 3 $\mathrm{HCs}, 4 \mathrm{CCs}$, nompC-Gal4, nompC ${ }^{3}$ background: $4 \mathrm{HCs}, 5 \mathrm{CCs}$,UAS-nompC nompC ${ }^{3}$ background: $5 \mathrm{HCs}, 4 \mathrm{CCs}$ 


\section{Chapter 7}

\section{List of abbreviations}

$\begin{array}{ll}\text { brv } & \text { brivido } \\ \text { CC } & \text { cold-cell } \\ \text { EGFP } & \text { enhanced green fluorescent protein } \\ \text { fps } & \text { frames per second } \\ \text { GCaMP6m } & \text { green fluorescent calmodulin M13 fusion protein } 6 \text { medium } \\ \text { GECI } & \text { genetically encoded calcium indicator } \\ \text { GFP } & \text { green fluorescent protein } \\ \text { gr28b.d } & \text { gustatory receptor 28b.d } \\ \text { GUI } & \text { graphical user interface } \\ \text { HC } & \text { hot-cell } \\ \text { IGLOO } & \text { Igloo is a Gradient Locomotion Model } \\ \text { md neurons } & \text { multidendritic neurons } \\ \text { nompC } & \text { no mechanoreceptor potential C } \\ \text { nSyb } & \text { neuronal synaptobrevin } \\ \text { PAL } & \text { posterior antennal lobe } \\ \text { PAP } & \text { proximal antennal protocerebrum } \\ \text { PI } & \text { preference index } \\ \text { PID controller } & \text { proportional-integral-derivative controller } \\ \text { ROI } & \text { region of interest } \\ \text { SEM } & \text { standard error of the mean } \\ \text { TRP channel } & \text { transient receptor potential channel } \\ \text { VNC } & \text { ventral nerve cord } \\ \text { wt } & \text { wild-type } \\ & \end{array}$




\section{Chapter 8}

\section{Acknowledgements}

First and foremost, I would like to thank Bart for his supervision and guidance during these past years. Without a doubt, noone else has taught me as much as you, be it about neuroscience, about coding, about how to approach scientific question or about how to present your work and yourself. You went far and beyond everything one could expect from a supervisor and I appreciate every conversation we had (and will have), ranging from topics about science to little plastic toys.

I want to thank Martin for his support during the entire project and also always having an open door and ear for problems and requests. I further thank Ernst Wimmer and Manuela Schmidt for being part of my thesis committee and providing constructive feedback and guidance throughout the project. I would like to extend my thanks to the members of the examination board, Roland Dosch, Jan Clemens and Tim Gollisch.

Daniela, I don't think I can thank you enough for all the support, patience and fun during these last years. There very few things that can cheer me up, when I'm really down, but somehow you always manage to do it. I could not have done this without you.

Furthermore I want to thank Nikoloz Sirmpilatze and Andrea Adden for preceding experiments on physiology and anatomy of the arista, thereby helping to establish the procedures of this study.

I want to thank Krissy and Diego for being the best lab-mates one can imagine. The times with you in- and outside the lab were always great, whether it was during scientific discussion or just laughing about stupid memes. Know that I consider you as more than just colleagues and more than just friends.

Special thanks also goes to Phil and Thomas for their constant nagging, I mean constructive feedback, especially during the last part of my thesis work. I am very grateful for all the times that Phil took some time to answer my (often stupid) questions about imaging, 
molecular biology and genetics.

And of course I thank all the remaining members (and former members) of the Department of Cellular Neurobiology, for making this work group the most welcoming and supportive work environment that I was ever a part of.

I would also like to thank Jan Hoffman, Tobias Mühmer and Sven Neumann for their constant and skillful help in building and fixing many of the setups that I worked on, and for always keeping up a good mood, no matter how annoying our requests became.

And of course thank you to my Mother and my sister Louise, for always being supportive and listening to all my ramblings and problems, no matter how small or big. I've experienced nothing but support for the way I chose, ever since I decided to up and leave our hometown.

To all who accompanied me these past years (and to those that I unjustly forgot to mention here) my deepest gratitude. 
Chapter 9

Curriculum Vitae 


\title{
Robert Kossen
}

\author{
Personal Information \\ Name: Robert Kossen \\ Date of birth March 4th, 1988 \\ Place of birth Bremen, Germany \\ Nationality German
}

\section{Education}

2015 - Present Georg-August-University Göttingen, PhD, Thesis Title:"Thermosensory Transduction Mechanisms in Drosophila melanogaster, Programme: Sensory and Motor Neuroscience.

Supervisor: Prof. Dr. Martin C. Göpfert, Thesis Committee Members: Prof. Dr. Ernst Wimmer, Prof. Dr. Manuela Schmidt

October 2011 - Georg-August-University Göttingen, Msc Neurobiology, Thesis Ti-

March 2014 tle:"Characterization of the T1-neuron in Drosophila melanogaster" , Programme: Developmental, Neural and Behavioural Biology.

Supervisors: Dr. Bart R.H. Geurten and Prof. Dr. Martin C. Göpfert

October 2007 - Georg-August-University Göttingen, Bsc Biology, Thesis Title:"Analyse sen-

May 2011 sorischer Mechanismen in der Haltere von Drosophila melanogaster " .

Supervisor: Prof. Dr. Martin C. Göpfert

\section{Publications}

Corthals, K., Heukamp, A.S., Kossen, R., Großhennig, I., Hahn, N., Gras, H., Göpfert, M.C., Heinrich, R., Geurten, B.R.H.

"Neuroligins NIg2 and Nlg4 Affect Social Behavior in Drosophila melanogaster"

Frontiers in Psychiatry, 8. https://doi.org/10.3389/fpsyt.2017.00113

\section{Teaching experience}

May 2019 Teacher Assistant in the practical course "Neurobiology II", MSc Developmental, Neural and Behavioural Biology, Georg-August-University Göttingen, Göttingen, Germany.

February - Teacher Assistant in the practical course "Tierphysiologie", BSc Biology

March 2018 Programme, Georg-August-University Göttingen, Göttingen, Germany.

February - Teacher Assistant in the practical course "Tierphysiologie", BSc Biology

March 2017 Programme, Georg-August-University Göttingen, Göttingen, Germany.

March 2016 Teacher Assistant in the practical course "Tierphysiologie", BSc Biology Programme, Georg-August-University Göttingen, Göttingen, Germany. 


\section{Awards and Scholarships}

2018 GGNB Travel grant. Awarded to participate in "Force gated Ion Channels Conference", 2018

2013 Stipend of the Otto-Vahlbruch-Foundation

\section{Conference contributions}

March 2019 13th Göttingen Meeting of the German Neuroscience Society, Poster presentation, Drosophila thermosensation is modulated by a mechano TRPchannel, Kossen, R., Adden, A., Giraldo, D., Göpfert, MC., Geurten, B.R.H.. Göttingen, Germany

October 2018 Flying Senses Syposium, Poster presentation, Thermosensory transduction in the antenna of Drosophila melanogaster, Kossen, R., Adden, A., Giraldo, D., Göpfert, MC., Geurten, B.R.H..

Göttingen, Germany

October 2018 Force Gated Ion Channels Conference, Poster presentation, Mechano-TRP modulates Drosophila heat sensation, Kossen, R., Adden, A., Giraldo, D., Göpfert, MC., Geurten, B.R.H.

Berlin, Germany

December 2017 H3 Symposium: Sensory Transduction in Insects, Poster presentation, Thermosensory transduction in the antenna of Drosophila melanogaster, Kossen, R., Adden, A., Göpfert, MC., Geurten, B.R.H..

London, UK

March 2017 12th Göttingen Meeting of the German Neuroscience Society, Poster presentation, Sex and Violence - Social Phenotypes in DNL2 and 4 deficient Drosophila, Kossen, R., Corthals, K., Heukamp, A.S., Großhennig, I., Hahn, N., Gras, H., Göpfert, M.C., Heinrich, R., Geurten, B.R.H..

Göttingen, Germany

August 2016 NeuroDoWo 2016, Poster presentation, Neuroligin-deficient Drosophila: Too dense or too deaf to mate?, Kossen, R., Corthals, K., Heukamp, A.S., Großhennig, I., Hahn, N., Gras, H., Göpfert, M.C., Heinrich, R., Geurten, B.R.H..

Bielefeld, Germany

Languages
English Fluent
French Basic

